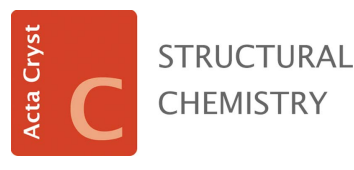

ISSN 2053-2296

\section{4-Styrylquinolines from cyclocondensation reactions between (2-aminophenyl)chalcones and 1,3-diketones: crystal structures and regiochemistry}

\author{
Diego Rodríguez, ${ }^{a}$ Sergio Andrés Guerrero, ${ }^{a}$ Alirio Palma, ${ }^{a}$ Justo Cobo ${ }^{b}$ and \\ Christopher Glidewell ${ }^{\mathrm{C} *}$
}

Received 3 August 2020

Accepted 4 August 2020

Edited by A. L. Spek, Utrecht University, The Netherlands

Keywords: synthesis; cyclocondensation; quinolone; crystal structure; molecular conformation; hydrogen bonding; supramolecular assembly; regiochemistry.

CCDC references: 2021528; 2021527; 2021526; 2021525; 2021524; 2021523

Supporting information: this article has supporting information at journals.iucr.org/C

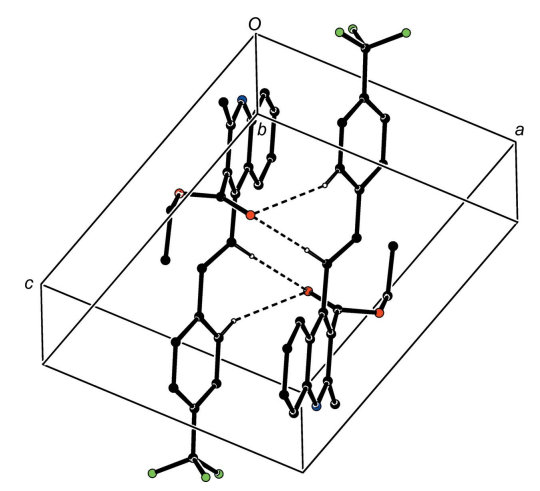

OPEN $\odot$ ACCESS
aLaboratorio de Síntesis Orgánica, Escuela de Química, Universidad Industrial de Santander, AA 678, Bucaramanga, Colombia, ${ }^{\mathbf{b}}$ Departamento de Química Inorgánica y Orgánica, Universidad de Jaén, 23071 Jaén, Spain, and ${ }^{\mathbf{C}}$ School of Chemistry, University of St Andrews, Fife KY16 9ST, Scotland. *Correspondence e-mail: cg@st-andrews.ac.uk

Structures are reported for two matched sets of substituted 4-styrylquinolines which were prepared by the formation of the heterocyclic ring in cyclocondensation reactions between 1-(2-aminophenyl)-3-arylprop-2-en-1-ones with 1,3-dicarbonyl compounds. (E)-3-Acetyl-4-[2-(4-methoxyphenyl)ethenyl]-2-methylquinoline, $\mathrm{C}_{21} \mathrm{H}_{19} \mathrm{NO}_{2}$, (I), (E)-3-acetyl-4-[2-(4-bromophenyl)ethenyl]-2-methylquinoline, $\mathrm{C}_{20} \mathrm{H}_{16} \mathrm{BrNO}$, (II), and (E)-3-acetyl-2-methyl-4-\{2-[4-(trifluoromethyl)phenyl]ethenyl\}quinoline, $\mathrm{C}_{21} \mathrm{H}_{16} \mathrm{~F}_{3} \mathrm{NO}$, (III), are isomorphous and in each structure the molecules are linked by a single $\mathrm{C}-\mathrm{H} \cdots \mathrm{O}$ hydrogen bond to form $C(6)$ chains. In (I), but not in (II) or (III), this is augmented by a C$\mathrm{H} \cdots \pi$ (arene) hydrogen bond to form a chain of rings; hence, (I)-(III) are not strictly isostructural. By contrast with (I)-(III), no two of ethyl (E)-4-[2-(4methoxyphenyl)ethenyl]-2-methylquinoline-3-carboxylate, $\mathrm{C}_{22} \mathrm{H}_{21} \mathrm{NO}_{3}$, (IV), ethyl (E)-4-[2-(4-bromophenyl)ethenyl]-2-methylquinoline-3-carboxylate, $\mathrm{C}_{21} \mathrm{H}_{18^{-}}$ $\mathrm{BrNO}_{2}$, (V), and ethyl (E)-2-methyl-4-\{2-[4-(trifluoromethyl)phenyl]ethenyl\}quinoline-3-carboxylate, $\mathrm{C}_{22} \mathrm{H}_{18} \mathrm{~F}_{3} \mathrm{NO}_{2}$, (VI), are isomorphous. The molecules of (IV) are linked by a single $\mathrm{C}-\mathrm{H} \cdots \mathrm{O}$ hydrogen bond to form $C(13)$ chains, but cyclic centrosymmetric dimers are formed in both (V) and (VI). The dimer in $(\mathrm{V})$ contains a $\mathrm{C}-\mathrm{H} \cdots \pi$ (pyridyl) hydrogen bond, while that in (VI) contains two independent $\mathrm{C}-\mathrm{H} \cdots \mathrm{O}$ hydrogen bonds. Comparisons are made with some related structures, and both the regiochemistry and the mechanism of the heterocyclic ring formation are discussed.

\section{Introduction}

Compounds containing 2-styrylquinoline units have attracted interest in recent years because of their potential as anticancer (El-Sayed et al., 2018), anti-HIV (Polanski et al., 2002), antimalarial (Roberts et al., 2017) and antimicrobial (Cieslik et al., 2012) agents, as well as in the treatment of Alzheimer's dementia (Wang et al., 2015). By contrast, analogous compounds containing 4-styryl units have been very much less extensively investigated, probably, at least in part, because of a lack of efficient and versatile methods for their synthesis: such methods have generally been based on coupling reactions requiring the prior synthesis of haloquinolines or (haloalkyl)quinolines, combined with either harsh reaction conditions or the use of expensive heavy-metal catalysts (Omar \& Hormi, 2009; Xia et al., 2016). However, a very straightforward synthesis of 4-styrylquinolines has been developed recently (Meléndez et al., 2020), in which the heterocyclic ring of the quinoline unit is built in situ using a cyclocondensation reaction between a $2^{\prime}$-aminochalcone, $(A)$, and a 1,3-dicarbonyl compound ( $c f$. Scheme 1). The chalcone component in this 
type of cyclization is readily accessible by reaction between 2 -aminoacetophenone and an aromatic aldehyde, allowing incorporation of a wide variety of substituents both in the styryl portion and at the 3-position of the quinoline nucleus. We report here the molecular structures and supramolecular assembly of two matched sets, each of three related products: the 3-acetyl derivatives, compounds (I)-(III) (Scheme 1 and Figs. 1-3), where $X=\mathrm{Me}$, were all obtained using pentane-2,4dione as the dicarbonyl component, while the 3-carboethoxy derivatives, compounds (IV)-(VI) (Figs. 4-6), where $X=\mathrm{OEt}$, were all obtained using ethyl 3-oxobutanoate (ethyl acetoacetate). Compounds such as (I)-(III), containing an acetyl

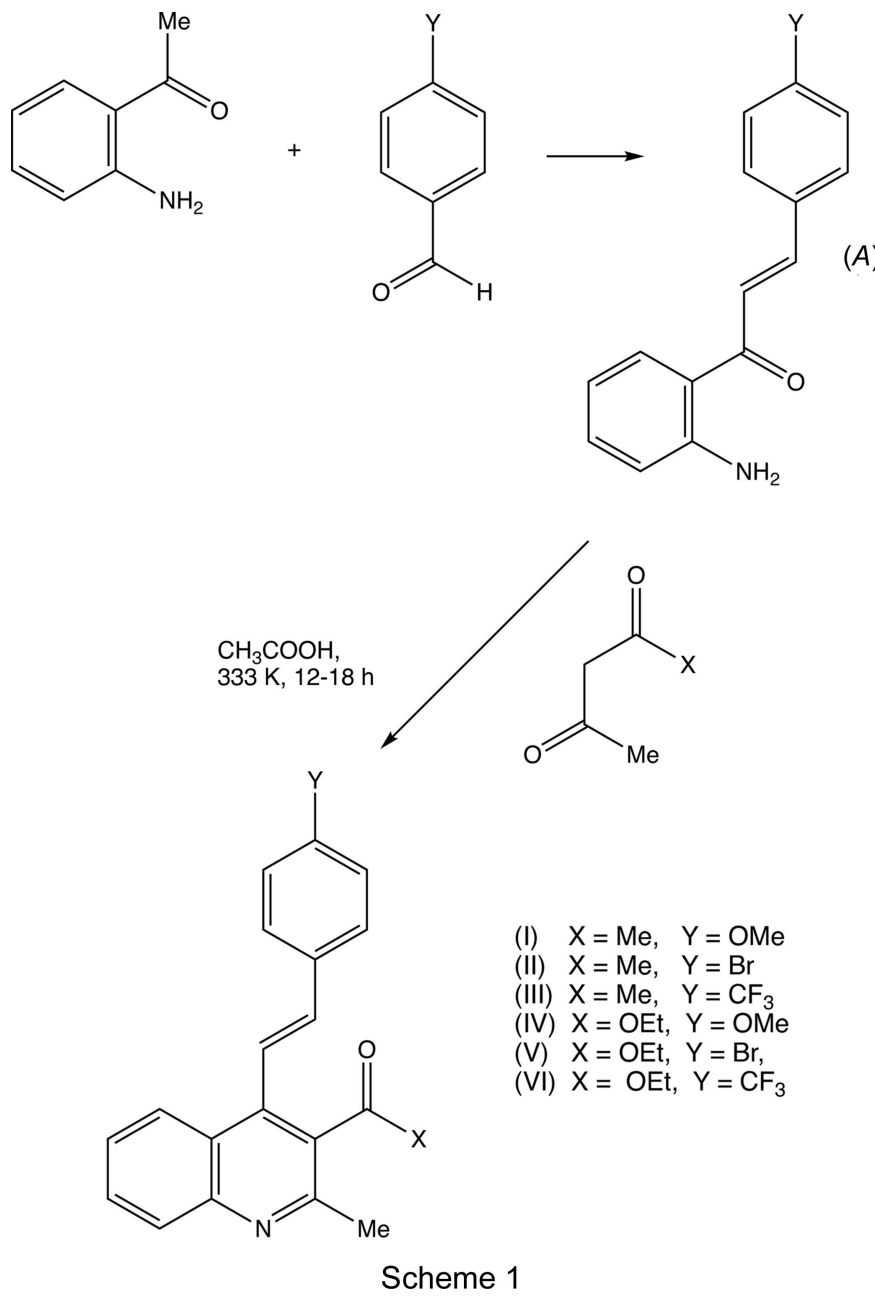

group, can act as useful synthetic intermediates, as they can undergo condensation with a further substituted aldehyde to form a chalcone substituent at the 3-position, as exemplified by compound (VIII) (Scheme 2). Such chalcones can themselves then undergo cyclocondensation reactions, for example, with a hydrazine, to form either a pyrazole, under basic conditions (Samshuddin et al., 2014), or a reduced pyrazole ring, under acidic conditions (Jasinski et al., 2010), or with guanidine to form a reduced pyrimidine ring (Nayak et al., 2014), thus giving access to a rich diversity of new 4-styrylquinolin-3-yl heterocycles. In addition to reporting the mol- ecular and supramolecular structures of compounds (I)-(VI), we also briefly consider compounds (VII) and (VIII) (Scheme 2). These have been reported on a simple proof of constitution basis [Cambridge Structural Database (CSD; Groom et al., 2016) refcodes MUMZEC and MUMZIG (Meléndez et al., 2020)] but without any structure description or discussion; accordingly, we discuss here the supramolecular assembly in these two compounds.<smiles>COc1ccc(/C=C/c2c(C(=O)/C=C/c3ccccc3)c(C)nc3ccccc23)cc1</smiles>

Scheme 2

\section{Experimental}

\subsection{Synthesis and crystallization}

Samples of compounds (I)-(VI) were prepared and crystallized following a recently published procedure (Meléndez et al., 2020).

\subsection{Refinement}

Crystal data, data collection and structure refinement details for compounds (I)-(VI) are summarized in Table 1. Two low-angle reflections which had been attenuated by the beam stop [100 for (I) and $\overline{1} 01$ for (VI)] were omitted from the data sets before the final refinements; likewise, two bad outlier reflections (639 and 606) were removed from the data set for (IV). All $\mathrm{H}$ atoms were located in difference maps and subsequently treated as riding atoms in geometrically idealized positions, with $\mathrm{C}-\mathrm{H}=0.95$ (alkenyl, aromatic and heteroaromatic), $0.98\left(\mathrm{CH}_{3}\right)$ or $0.99 \AA\left(\mathrm{CH}_{2}\right)$, and with 
Table 1

Experimental details.

Experiments were carried out at $100 \mathrm{~K}$ with Mo K $\alpha$ radiation using a Bruker D8 Venture diffractometer. Absorption was corrected for by multi-scan methods (SADABS; Bruker, 2016). H-atom parameters were constrained.

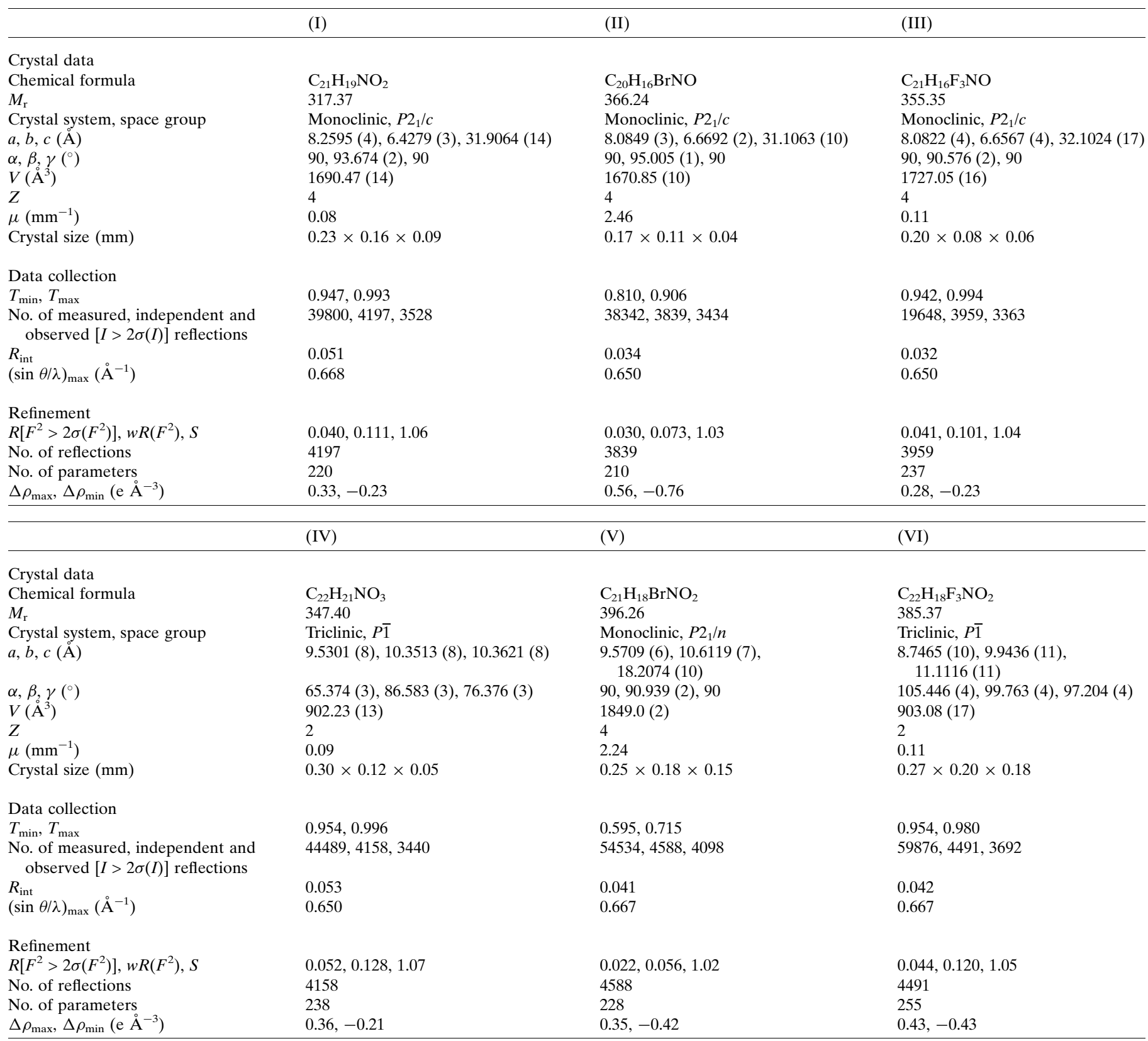

Computer programs: APEX3 (Bruker, 2018), SAINT (Bruker, 2017), SHELXT2014 (Sheldrick, 2015a), SHELXL2014 (Sheldrick, 2015b) and PLATON (Spek, 2020).

$U_{\text {iso }}(\mathrm{H})=k U_{\text {eq }}(\mathrm{C})$, where $k=1.5$ for the methyl groups, which were permitted to rotate but not to tilt, and 1.2 for all other $\mathrm{H}$ atoms. For compounds (VII) and (VIII), the published structures (Meléndez et al., 2020) were inverted and the atom labelling adjusted slightly in order to bring them into full conformity with compounds (I)-(VI) ( $c f$. Tables 2 and 3); the modified versions of the CIFs for (VII) and (VIII) are provided in the supporting information. Examination of the structure for (VIII) using PLATON (Spek, 2020) showed that the unit cell contains two voids, each of volume $60 \AA^{3}$ and centred at $\left(0, \frac{1}{2}, 0\right)$ and $\left(\frac{1}{2}, 0, \frac{1}{2}\right)$, but further examination using
SQUEEZE (Spek, 2015) showed that these voids contained negligible electron density.

\section{Results and discussion}

In reactions between a chalcone of type $(A)$ (Scheme 1) and a symmetrical 1,3-diketone, such as pentane-2,4-dione, only a single product is possible, namely, the 3-acetyl-2-methylquinoline derivative, as exemplified by compounds (I)-(III). However, a comparable reaction involving an unsymmetrical diketone, such as 1-phenylbutane-1,3-dione can give two 


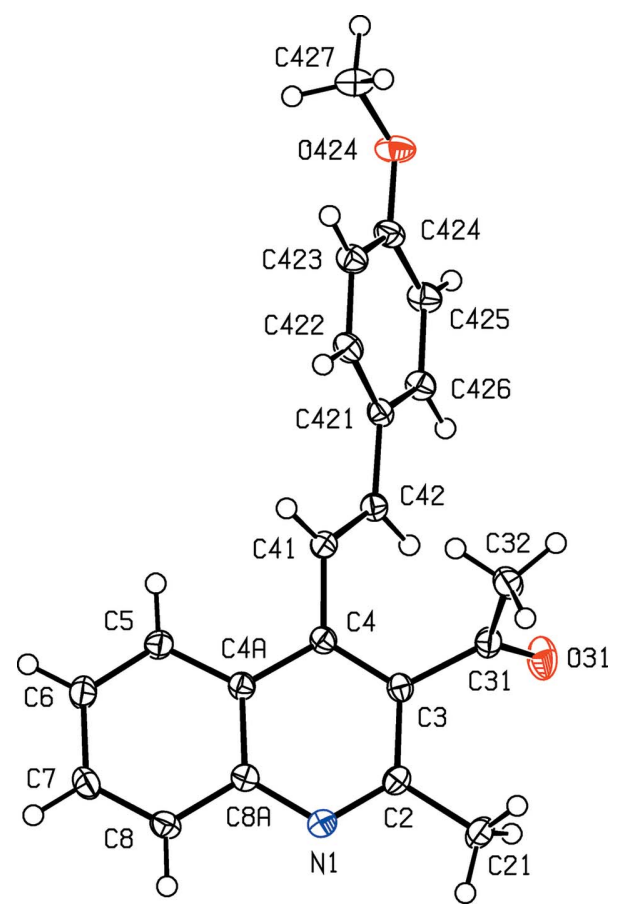

Figure 1

The molecular structure of compound (I), showing the atom-labelling scheme. Displacement ellipsoids are drawn at the $50 \%$ probability level.

regioisomers, such as (VII), if the amino group reacts at the acetyl carbonyl group, or the alternative (IX) if the reaction occurs at the benzoyl carbonyl group. In general, reactions with this ketone lead exclusively to the 3-benzoyl-2-methyl isomers, as exemplified by (VII), rather than to the 3-acetyl-2phenyl alternative exemplified by (IX) (Meléndez et al., 2020),

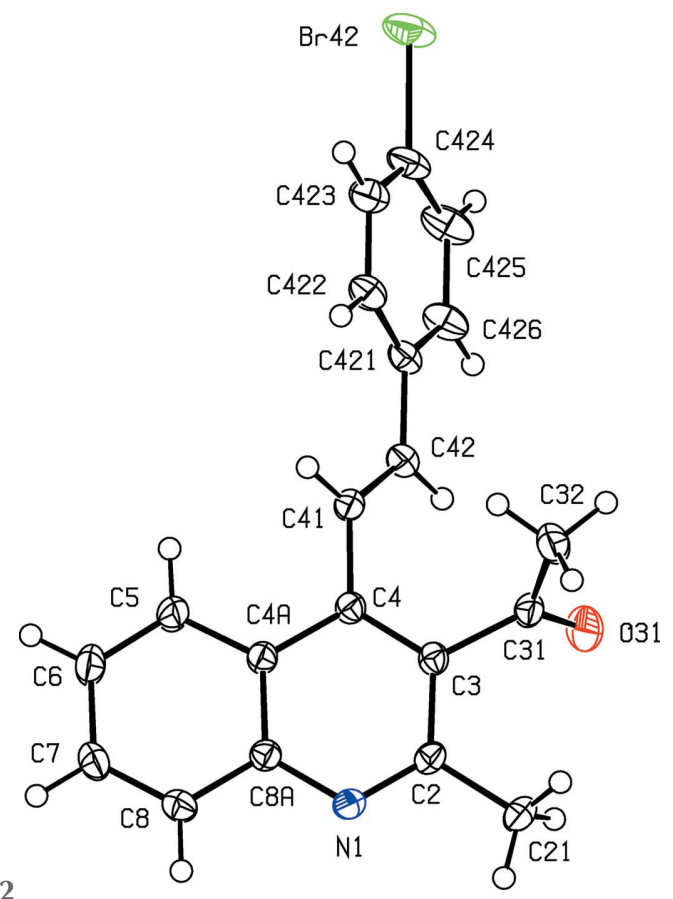

Figure 2

The molecular structure of compound (II), showing the atom-labelling scheme. Displacement ellipsoids are drawn at the $50 \%$ probability level.

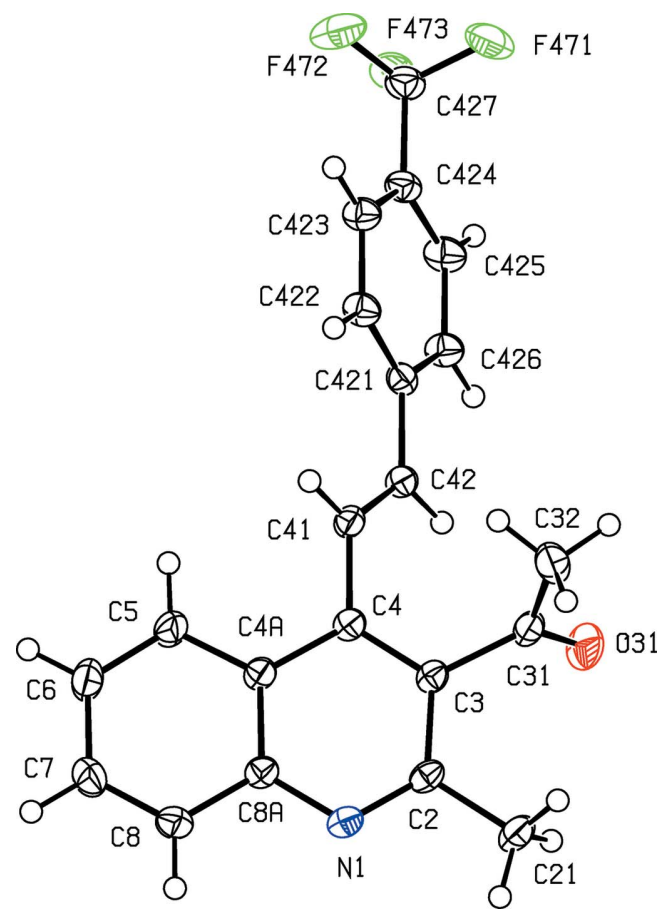

Figure 3

The molecular structure of compound (III), showing the atom-labelling scheme. Displacement ellipsoids are drawn at the $50 \%$ probability level.

which is consistent with the greater reactivity in the nucleophilic addition reaction of acetyl groups compared with benzoyl groups (Bürgi et al., 1974; Katritzsky et al., 1995; Meléndez et al., 2020). Similarly, the reaction of a chalcone of type $(A)$ with an unsymmetrical diketo compound, such as

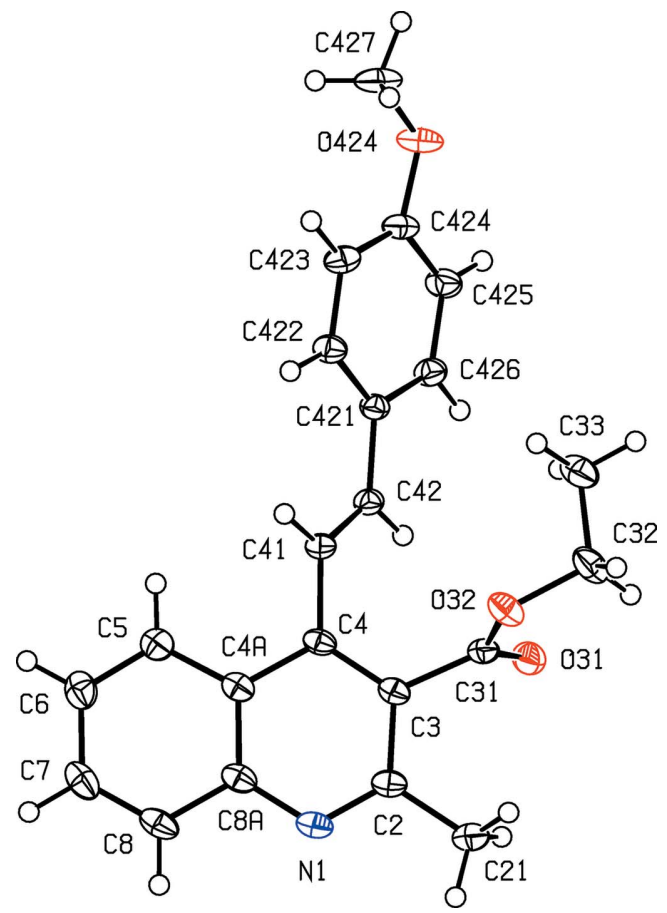

Figure 4

The molecular structure of compound (IV), showing the atom-labelling scheme. Displacement ellipsoids are drawn at the $50 \%$ probability level. 
Table 2

Selected torsion angles $\left({ }^{\circ}\right)$ for compounds (I)-(VIII).

\begin{tabular}{lllcr}
\hline Compound & $\begin{array}{llll}\mathrm{C} 3-\mathrm{C} 4- \\
\mathrm{C} 41-\mathrm{C} 42\end{array}$ & \multicolumn{1}{c}{$\mathrm{C} 41-\mathrm{C} 42-$} & $\mathrm{C} 2-\mathrm{C} 3-$ & $\mathrm{C} 2-\mathrm{C} 3-$ \\
(I) & $46.40(16)$ & $13.78(18)$ & $66.18(15)$ & \\
(II) & $46.8(3)$ & $14.8(3)$ & $68.2(2)$ & \\
(III) & $49.0(2)$ & $13.0(2)$ & $69.52(18)$ & \\
(IV) & $48.8(2)$ & $1.2(3)$ & $73.7(2)$ & $-104.85(17)$ \\
(V) & $51.4(2)$ & $10.6(2)$ & $-102.56(17)$ & $75.88(15)$ \\
(VI) & $34.8(2)$ & $-17.7(2)$ & $-99.75(16)$ & $76.46(15)$ \\
(VII) & $51.0(5)$ & $2.0(5)$ & $71.5(4)$ & \\
(VIII) & $54.17(19)$ & $-4.0(2)$ & $-97.59(15)$ & \\
\hline
\end{tabular}

ethyl 3-oxobutanoate, can, in principle, give two types of product: reaction of the amino group at the acetyl carbonyl group leads to ethyl esters, as exemplified by compounds (IV)-(VI), but reaction of the amino group at the ester carbonyl group would lead to elimination of ethanol with the formation of a 2-quinolone of type (X) (Scheme 2). Again, these reactions appear to lead exclusively to the esters, as exemplified by (IV)-(VI) (Meléndez et al., 2020), consistent with the greater electrophilicity of a ketonic carbonyl group compared with an ester carbonyl group. On the other hand, 2-aryl-4-quinolones are sometimes formed as by-products arising from an intramolecular cyclization of the chalcone precursor.

Compounds (I)-(III), where $X=\mathrm{Me}$ and $Y=\mathrm{OMe}, \mathrm{Br}$ or $\mathrm{CF}_{3}$, respectively (Scheme 1 and Figs. 1-3), all crystallize in the space group $P 22_{1} / c$ with rather similar unit-cell dimensions (Table 1) and very similar molecular conformations (Table 2); each structure can be refined using the coordinates of one of

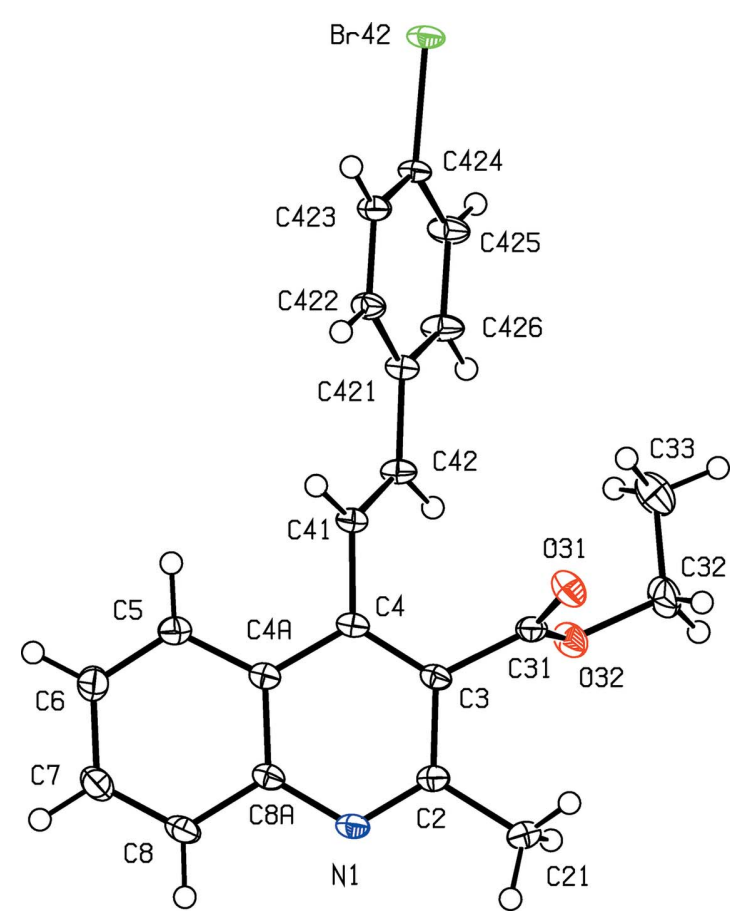

Figure 5

The molecular structure of compound (V), showing the atom-labelling scheme. Displacement ellipsoids are drawn at the $50 \%$ probability level.

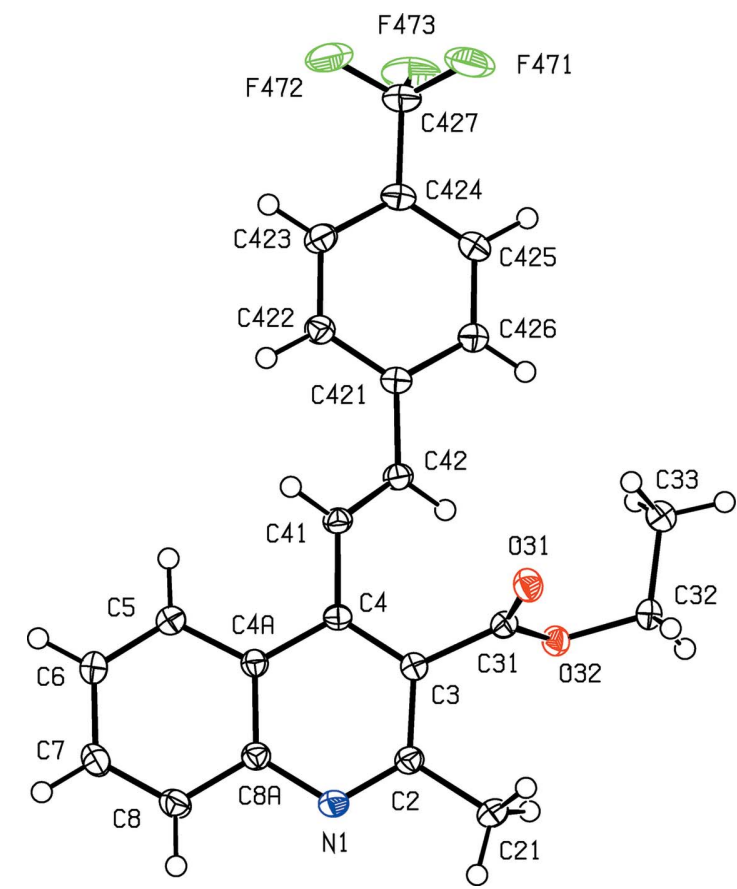

Figure 6

The molecular structure of compound (VI), showing the atom-labelling scheme. Displacement ellipsoids are drawn at the $50 \%$ probability level.

the others as the starting point, provided due alteration is made in the substituent at atom C424 (Figs. 1-3). However, although there are short intermolecular $\mathrm{C}-\mathrm{H} \cdots \mathrm{O}$ and $\mathrm{C}-$ $\mathrm{H} \cdots \pi$ (arene) contacts in all three compounds, involving the same sets of atoms (Table 3), in each of compounds (II) and

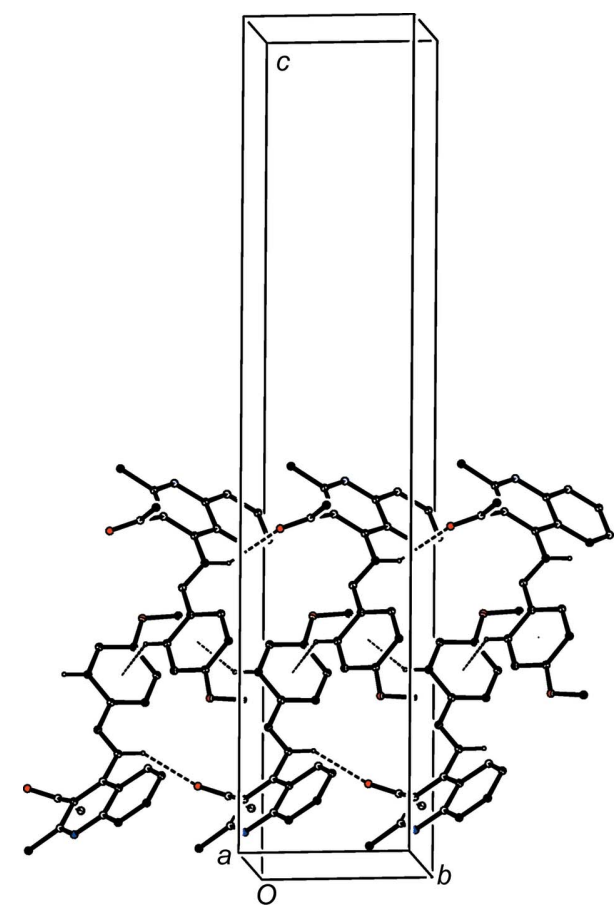

Figure 7

Part of the crystal structure of compound (I), showing the formation of a chain of rings along [010] built from $\mathrm{C}-\mathrm{H} \cdots \mathrm{O}$ and $\mathrm{C}-\mathrm{H} \cdots \pi$ (arene) hydrogen bonds, drawn as dashed lines. For the sake of clarity, $\mathrm{H}$ atoms not involved in the motifs shown have been omitted. 
Table 3

Hydrogen bonds and short intramolecular contacts $\left(\AA{ }^{\circ}\right)$ for compounds (I)-(VIII)

$C g 1, C g 3, C g 4$ and $C g 5$ represent the centroids of the $\mathrm{N} 1 / \mathrm{C} 2-\mathrm{C} 4 / \mathrm{C} 4 A / \mathrm{C} 8 A$, C421-C426, C311-C316 [present in (VII) only] and C331-C336 [present in (VIII) only] rings, respectively; ring 2 comprises atoms $\mathrm{C} 4 A / \mathrm{C} 5-\mathrm{C} 8 / \mathrm{C} 8 A$.

\begin{tabular}{|c|c|c|c|c|c|}
\hline & $D-\mathrm{H} \cdots A$ & $D-\mathrm{H}$ & $\mathrm{H} \cdots A$ & $D \cdots A$ & $D-\mathrm{H} \cdots A$ \\
\hline \multirow[t]{2}{*}{ (I) } & $\mathrm{C} 41-\mathrm{H} 41 \cdots \mathrm{O} 31^{\mathrm{i}}$ & 0.95 & 2.41 & $3.2527(15)$ & 148 \\
\hline & $\mathrm{C} 426-\mathrm{H} 426 \cdots C g 3^{\mathrm{ii}}$ & 0.95 & 2.77 & $3.5252(13)$ & 138 \\
\hline \multirow[t]{2}{*}{ (II) } & $\mathrm{C} 41-\mathrm{H} 41 \cdots \mathrm{O} 31^{\mathrm{i}}$ & 0.95 & 2.37 & $3.283(2)$ & 161 \\
\hline & $\mathrm{C} 426-\mathrm{H} 426 \cdots C g 3^{\mathrm{ii}}$ & 0.95 & 2.91 & $3.7071(19)$ & 142 \\
\hline \multirow[t]{2}{*}{ (III) } & $\mathrm{C} 41-\mathrm{H} 41 \cdots \mathrm{O} 31^{\mathrm{i}}$ & 0.95 & 2.42 & 3.3290 (17) & 161 \\
\hline & $\mathrm{C} 426-\mathrm{H} 426 \cdots C g 3^{\mathrm{ii}}$ & 0.95 & 3.00 & $3.7621(15)$ & 138 \\
\hline \multirow[t]{2}{*}{ (IV) } & $\mathrm{C} 32-\mathrm{H} 32 B \cdots \mathrm{O} 424^{\mathrm{iii}}$ & 0.99 & 2.57 & $3.406(2)$ & 142 \\
\hline & $\mathrm{C} 423-\mathrm{H} 423 \cdots C g 1^{\mathrm{iv}}$ & 0.95 & 2.91 & 3.4894 (19) & 120 \\
\hline \multirow[t]{2}{*}{ (V) } & $\mathrm{C} 423-\mathrm{H} 423 \cdots \mathrm{O} 31^{\mathrm{v}}$ & 0.95 & 2.59 & 3.2197 (17) & 124 \\
\hline & $\mathrm{C} 426-\mathrm{H} 426 \cdots C g 1^{\text {iv }}$ & 0.95 & 2.74 & $3.5961(16)$ & 151 \\
\hline \multirow[t]{2}{*}{ (VI) } & $\mathrm{C} 41-\mathrm{H} 41 \cdots \mathrm{O} 31^{\mathrm{iv}}$ & 0.95 & 2.54 & $3.4922(18)$ & 178 \\
\hline & $\mathrm{C} 422-\mathrm{H} 422 \cdots \mathrm{O} 31^{\mathrm{iv}}$ & 0.95 & 2.56 & 3.3924 (19) & 146 \\
\hline \multirow[t]{3}{*}{ (VII) } & $\mathrm{C} 41-\mathrm{H} 41 \cdots \mathrm{O} 31^{\mathrm{vi}}$ & 0.95 & 2.37 & $3.300(5)$ & 167 \\
\hline & $\mathrm{C} 422-\mathrm{H} 422 \cdots \mathrm{O} 31^{\mathrm{vi}}$ & 0.95 & 2.57 & $3.506(4)$ & 169 \\
\hline & $\mathrm{C} 426-\mathrm{H} 426 \cdots C g 4^{\mathrm{vii}}$ & 0.95 & 2.67 & 3.549 (4) & 155 \\
\hline \multirow[t]{3}{*}{ (VIII) } & $\mathrm{C} 334-\mathrm{H} 334 \cdots \mathrm{O} 31^{\text {viii }}$ & 0.95 & 2.61 & $3.542(2)$ & 168 \\
\hline & $\mathrm{C} 7-\mathrm{H} 7 \cdots C g 5^{\mathrm{ix}}$ & 0.95 & 2.93 & $3.6437(16)$ & 133 \\
\hline & $\mathrm{C} 422-\mathrm{H} 422 \cdots C g 1^{\mathrm{x}}$ & 0.95 & 2.93 & $3.6300(17)$ & 132 \\
\hline
\end{tabular}

Symmetry codes: (i) $x, y+1, z$; (ii) $-x+1, y-\frac{1}{2},-z+\frac{1}{2}$; (iii) $x+1, y, z$; (iv) $-x+1,-y+1$, $-z+1$; (v) $-x+\frac{3}{2}, y+\frac{1}{2},-z+\frac{3}{2}$; (vi) $x-\frac{1}{2}, y,-z+\frac{1}{2}$; (vii) $-x+\frac{3}{2}, y-\frac{1}{2}, z$; (viii) $x-1, y, z$; (ix) $x+\frac{1}{2},-y+\frac{1}{2}, z-\frac{1}{2} ;(x) x+\frac{1}{2},-y+\frac{1}{2}, z+\frac{1}{2}$.

(III), the $\mathrm{H} \cdots C g$ distance is quite long and probably of marginal structural significance, whereas it can be regarded as a genuine hydrogen bond in compound (I). On this basis, compounds (I)-(III) can be regarded as isomorphous but not

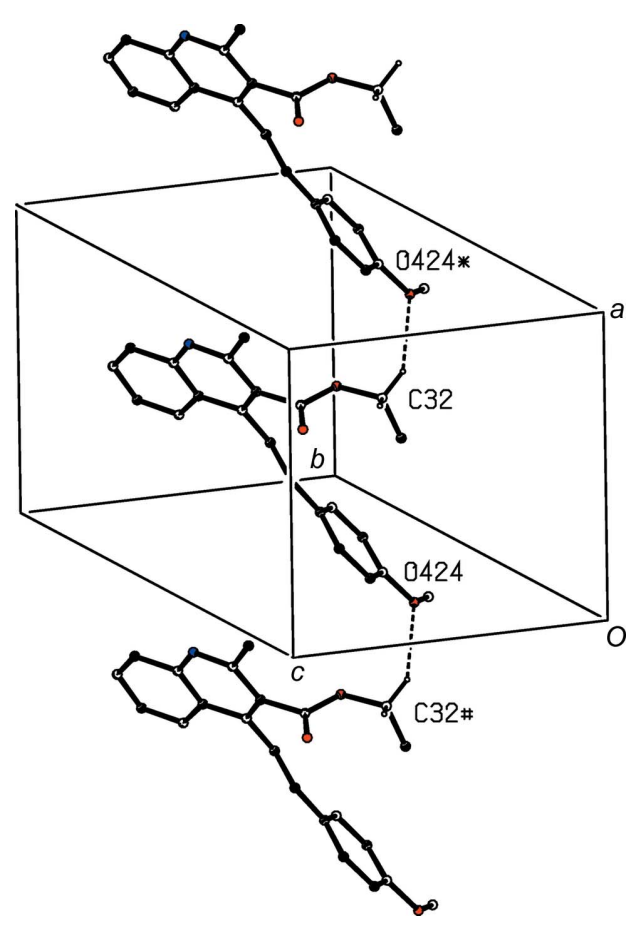

Figure 8

Part of the crystal structure of compound (IV), showing the formation of a $C(13)$ chain running parallel to the [100] direction. Hydrogen bonds are drawn as dashed lines and, for the sake of clarity, $\mathrm{H}$ atoms bonded to those $\mathrm{C}$ atoms which are not involved in the motif shown have been omitted. Atoms marked with an asterisk (*) or a hash (\#) are at the symmetry positions $(x+1, y, z)$ and $(x-1, y, z)$, respectively. strictly isostructural (Acosta et al., 2009; Blanco et al., 2012). However, in the comparable series of compounds, i.e. (IV)(VI), where $X=\mathrm{OEt}$, although compounds (IV) and (VI) are both triclinic, in (IV) the inter-axial angles are all less than $90^{\circ}$, but in (VI) they are greater than $90^{\circ}$, so that these two compounds are far from being isomorphous. On the other hand, the third member of this group, compound $(\mathrm{V})$, is monoclinic, so there can be no close similarities within this group.

None of the molecules of (I)-(VIII) exhibits any internal symmetry, so that they are all conformationally chiral; in each case, the reference molecule was selected as one having a positive sign for the $\mathrm{C} 3-\mathrm{C} 4-\mathrm{C} 41-\mathrm{C} 42$ torsion angle (Table 2), although the space groups confirm that all the compounds have crystallized as conformational racemates.

The supramolecular assembly of compounds (I)-(VI) is determined by $\mathrm{C}-\mathrm{H} \cdots \mathrm{O}$ and $\mathrm{C}-\mathrm{H} \cdots \pi$ hydrogen bonds (Table 3). In each of (I)-(III), molecules which are related by translation are linked by a $\mathrm{C}-\mathrm{H}$. . O hydrogen bond to form a C(6) (Etter, 1990; Etter et al., 1990; Bernstein et al., 1995) chain running parallel to the [010] direction. In the structure of (I), this is enhanced by a $\mathrm{C}-\mathrm{H} \cdots \pi$ (arene) hydrogen bond linking molecules related by the $2_{1}$ screw axis along $\left(\frac{1}{2}, y, \frac{1}{4}\right)$ to form a chain of rings (Fig. 7). However, in the structures of (II) and (III), the corresponding $\mathrm{H} \cdots C g$ and $\mathrm{C} \cdots C g$ distances are much longer than they are in (I), so that these are possibly better regarded as short adventitious contacts rather than structurally significant hydrogen bonds.

A single $\mathrm{C}-\mathrm{H} \cdots \mathrm{O}$ hydrogen bond links the molecules of compound (IV) which are related by translation into a $C(13)$ chain running parallel to the [100] direction (Fig. 8). This

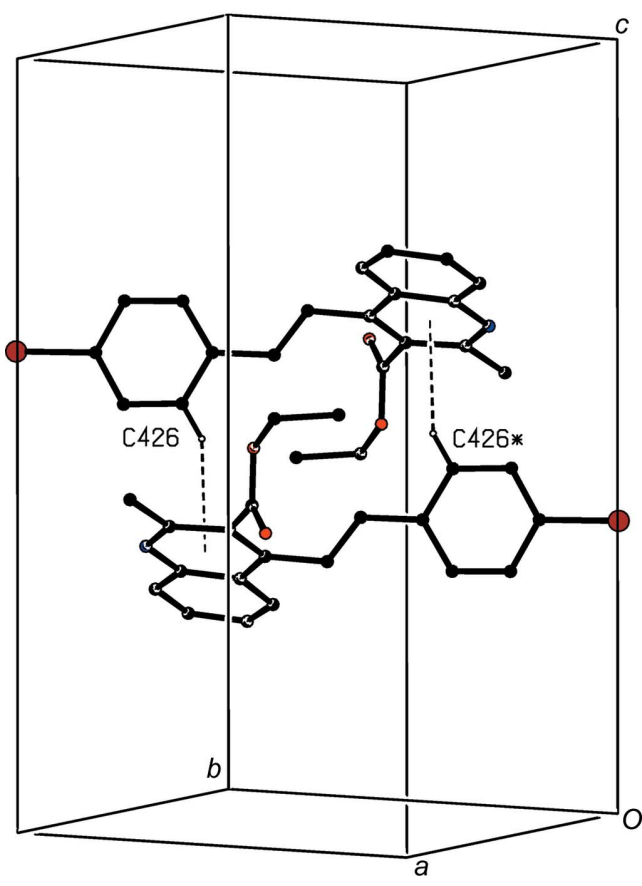

Figure 9

Part of the crystal structure of compound (V), showing the formation of a centrosymmetric dimer. Hydrogen bonds are drawn as dashed lines and, for the sake of clarity, $\mathrm{H}$ atoms which are not involved in the motif shown have been omitted. The atom marked with an asterisk (*) is at the symmetry position $(-x+1,-y+1,-z+1)$. 


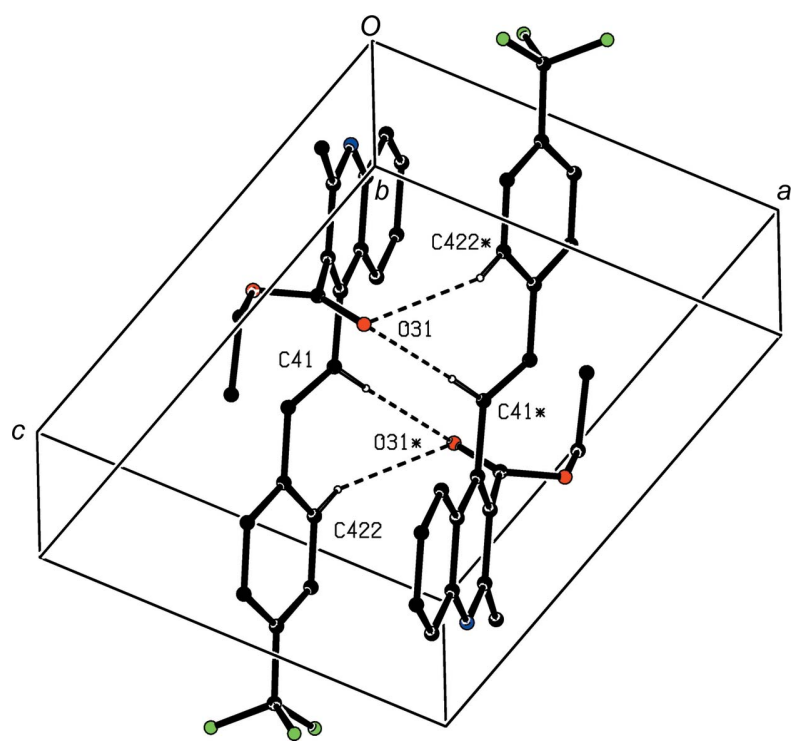

Figure 10

Part of the crystal structure of compound (VI), showing the formation of a centrosymmetric dimer. Hydrogen bonds are drawn as dashed lines and, for the sake of clarity, $\mathrm{H}$ atoms which are not involved in the motif shown have been omitted. Atoms marked with an asterisk $(*)$ are at the symmetry position $(-x+1,-y+1,-z+1)$.

structure also contains a short $\mathrm{C}-\mathrm{H} \cdots \pi$ (pyridyl) contact, but the long $\mathrm{H} \cdots C g$ distance and the very small $\mathrm{C}-\mathrm{H} \cdots C g$ angle indicate that this is probably not structurally significant (Wood et al., 2009). By contrast, in the structure of compound (V), it is the $\mathrm{C}-\mathrm{H} \cdots \mathrm{O}$ contact which has a very small $D-\mathrm{H} \cdots A$ angle (Table 3 ), while a $\mathrm{C}-\mathrm{H} \cdots \pi$ (pyridyl) hydrogen bond links

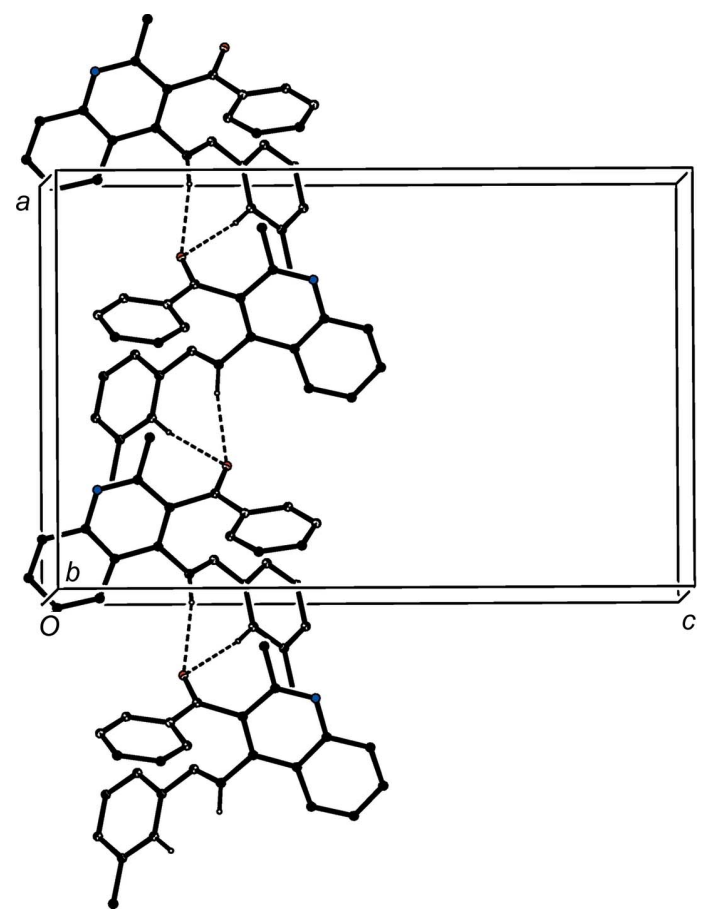

Figure 11

Part of the crystal structure of compound (VII), showing the formation of a $C(6) C(9)\left[R_{2}^{1}(7)\right]$ chain of rings running parallel to the [100] direction. Hydrogen bonds are drawn as dashed lines and, for the sake of clarity, $\mathrm{H}$ atoms which are not involved in the motif shown have been omitted.

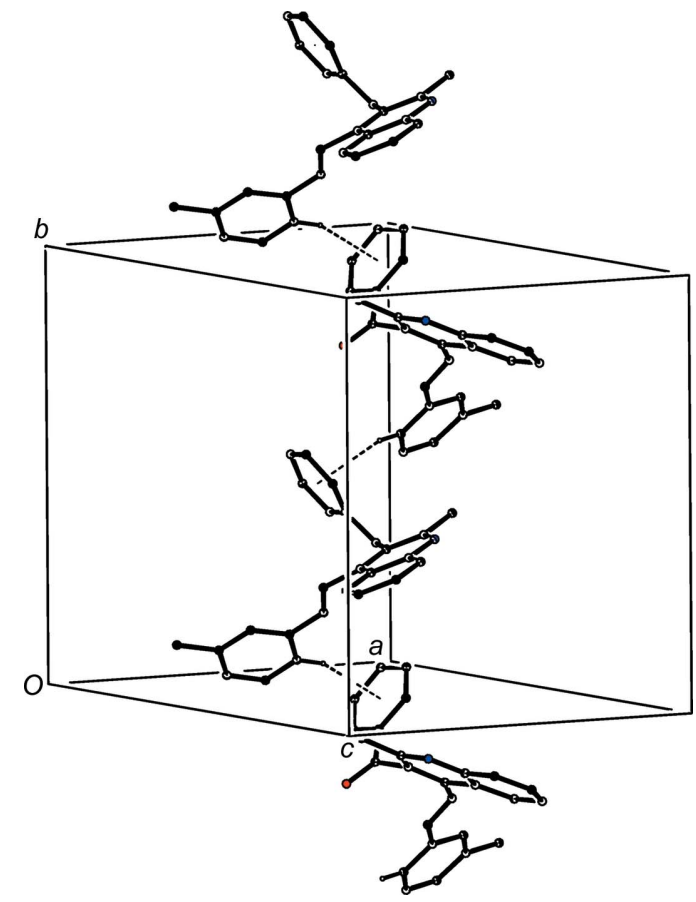

Figure 12

Part of the crystal structure of compound (VII), showing the formation of a chain built from $\mathrm{C}-\mathrm{H} \cdots \pi$ (arene) hydrogen bonds, drawn as dashed lines, running parallel to the [010] direction. For the sake of clarity, $\mathrm{H}$ atoms which are not involved in the motif shown have been omitted.

molecules which are related by inversion to form a cyclic centrosymmetric dimer (Fig. 9).

In the structure of compound (VI), there are no $\mathrm{C}-\mathrm{H} \cdots \pi$ hydrogen bonds or short intermolecular contacts. Instead two $\mathrm{C}-\mathrm{H} \cdots \mathrm{O}$ hydrogen bonds combine to link inversion-related pairs of molecules into centrosymmetric dimers. The hydrogen bonds involving atoms of type C41 form an $R_{2}^{2}(12)$ ring, while those involving atoms of type $\mathrm{C} 422$ generate an $R_{2}^{2}(18)$ ring (Fig. 10).

We also discuss here the supramolecular assembly of compounds (VII) and (VIII), which, as noted above ( $\$ 1$, Introduction), have been reported on a simple proof of constitution basis, without discussion (Meléndez et al., 2020). The assembly in (VII) in the space group Pbcn is based upon two $\mathrm{C}-\mathrm{H}$... O hydrogen bonds and one $\mathrm{C}-\mathrm{H} \cdots \pi$ (arene) hydrogen bond (Table 3). The two $\mathrm{C}-\mathrm{H}$. . O hydrogen bonds link molecules which are related by the $a$-glide plane at $z=\frac{1}{4}$ to form a $C(6) C(9)\left[R_{2}^{1}(7)\right]$ chain of rings running parallel to the [100] direction (Fig. 11). In addition, the structure of (VII) contains a $\mathrm{C}-\mathrm{H} \cdots \pi$ (arene) hydrogen bond which links molecules which are related by the $b$-glide plane at $x=\frac{3}{4}$ to form a chain running parallel to the [010] direction (Fig. 12). The combination of the chain motifs along [100] and [010] generates a complex sheet lying parallel to (001) in the domain $0<z<\frac{1}{2}$; a second sheet of this type, related to the first by inversion, lies in the domain $\frac{1}{2}<z<1.0$, but there are no direction-specific interactions between adjacent sheets. Even the shortest intermolecular contacts (Table 3) in chalcone (VIII) have $\mathrm{H} \cdots A$ distances which are probably too long for these contacts to be regarded as structurally significant. 
The structures of several simple 2-styrylquinolines have been published, including those of the unsubstituted 2-styrylquinoline itself (Valle et al., 1986), and of several analogues carrying simple substituents in the phenyl ring (Kuz'mina et al., 2012). In addition, structures have been reported for a number of salts derived from 2-styrylquinolines (Kobkeatthawin et al., 2008, 2009; Chantrapromma et al., 2008, 2014; Fun et al., 2013). For all of these compounds, the styryl group was introduced into a preformed quinoline nucleus. 8-Styrylquinoline and its 4-phenylstyryl analogue, whose structures have also been reported (Sharma et al., 2015), were prepared using a rhodiumcatalysed coupling reaction between quinoline $N$-oxide and the styrene component. Despite the substantial number of structure reports involving 2-styrylquinolines and their derivatives, there are no reports in the CSD of 4-styrylquinolines other than the two examples discussed above, i.e. compounds (VII) and (VIII) (CSD refcodes MUMZEC and MUMZIG, respectively; Meléndez et al., 2020).

\section{Acknowledgements}

The authors thank the Centro de Instrumentación, CientíficoTécnica of the Universidad de Jaén (UJA), and its staff for the data collection.

\section{Funding information}

Funding for this research was provided by: Vicerrectoría de Investigación y Extensión de la Universidad Industrial de Santander (proyecto No. 2497, to AP); Universidad de Jaén, the Consejería de Economía, Innovación, Ciencia y Empleo (Junta de Andalucía, Spain) and Centro de Instrumentación Científico-Técnica of the Universidad de Jaén (UJA) (to JC).

\section{References}

Acosta, L. M., Bahsas, A., Palma, A., Cobo, J., Hursthouse, M. B. \& Glidewell, C. (2009). Acta Cryst. C65, o92-o96.

Bernstein, J., Davis, R. E., Shimoni, L. \& Chang, N.-L. (1995). Angew. Chem. Int. Ed. Engl. 34, 1555-1573.

Blanco, M. C., Palma, A., Cobo, J. \& Glidewell, C. (2012). Acta Cryst. C68, 0195-o198.

Bruker (2016). SADABS. Bruker AXS Inc., Madison, Wisconsin, USA.

Bruker (2017). SAINT. Bruker AXS Inc., Madison, Wisconsin, USA.

Bruker (2018). APEX3. Bruker AXS Inc., Madison, Wisconsin, USA.

Bürgi, H. B., Dunitz, J. D., Lehn, J. M. \& Wipff, M. (1974). Tetrahedron, 30, 1563-1572.

Chantrapromma, S., Kaewmanee, N., Boonnak, N., Quah, C. K. \& Fun, H.-K. (2014). Acta Cryst. E70, o395-o396.
Chantrapromma, S., Kobkeatthawin, T., Chanawanno, K., Karalai, S. \& Fun, H.-K. (2008). Acta Cryst. E64, o876-o877.

Cieslik, W., Musiol, R., Nycz, J. E., Jampilek, J., Vejsova, M., Wolff, M., Machura, B. \& Polanski, J. (2012). Bioorg. Med. Chem. 20, 6960-6968.

El-Sayed, M. A.-A., El-Husseiny, W. M., Abdel-Aziz, N. I., El-Azab, A. S., Abuelizz, H. A. \& Abdel-Aziz, A. A.-M. (2018). J. Enzyme Inhib. Med. Chem. 33, 199-209.

Etter, M. C. (1990). Acc. Chem. Res. 23, 120-126.

Etter, M. C., MacDonald, J. C. \& Bernstein, J. (1990). Acta Cryst. B46, 256-262.

Fun, H.-K., Kaewmanee, N., Chanawanno, K., Boonnak, N. \& Chantrapromma, S. (2013). Acta Cryst. E69, o1510-o1511.

Groom, C. R., Bruno, I. J., Lightfoot, M. P. \& Ward, S. C. (2016). Acta Cryst. B72, 171-179.

Jasinski, J. P., Guild, C. J., Samshuddin, S., Narayana, B. \& Yathirajan, H. S. (2010). Acta Cryst. E66, o1948-o1949.

Katritzsky, A. R., Meth-Cohn, O. \& Rees, C. (1995). In Comprehensive Organic Functional Group Transformations. Oxford: Pergamon Press.

Kobkeatthawin, T., Ruanwas, P., Chantrapromma, S. \& Fun, H.-K. (2008). Acta Cryst. E64, o642-o643.

Kobkeatthawin, T., Suwunwong, T., Chantrapromma, S. \& Fun, H.-K. (2009). Acta Cryst. E65, o76-o77.

Kuz'mina, L. G., Sitin, A. G., Gulakova, E., Fedorova, O. A., Lermontova, E. K. \& Churakov, A. V. (2012). Crystallogr. Rep. 57, 85-95.

Meléndez, A., Plata, A., Rodríguez, R., Ardila, D., Guerrero, S. A., Acosta, L. M., Cobo, J., Nogueras, M. \& Palma, A. (2020). Synthesis, 52, 1804-1822.

Nayak, P. S., Narayana, B., Yathirajan, H. S., Hosten, E. C., Betz, R. \& Glidewell, C. (2014). Acta Cryst. C70, 1011-1016.

Omar, W. A. E. \& Hormi, O. E. O. (2009). Tetrahedron, 65, 44224428.

Polanski, J., Zouhiri, F., Jeanson, L., Desmaële, D., d'Angelo, J., Mouscadet, J. F., Gieleciak, R., Gasteiger, J. \& Le Bret, M. (2002). J. Med. Chem. 45, 4647-4654.

Roberts, B. F., Zheng, Y., Cleaveleand, J., Lee, S., Lee, E., Ayong, L., Yuan, Y. \& Chakrabarti, D. (2017). Int. J. Parasitol. Drugs Drug Resist. 7, 120-129.

Samshuddin, S., Jasinski, J. P., Golen, J. A., Narayana, B., Yathirajan, H. S. \& Glidewell, C. (2014). Acta Cryst. C70, 867-871.

Sharma, R., Kumar, R., Kumar, I. \& Sharma, U. (2015). Eur. J. Org. Chem. 2015, 7519-7528.

Sheldrick, G. M. (2015a). Acta Cryst. A71, 3-8.

Sheldrick, G. M. (2015b). Acta Cryst. C71, 3-8.

Spek, A. L. (2015). Acta Cryst. C71, 9-18.

Spek, A. L. (2020). Acta Cryst. E76, 1-11.

Valle, G., Busetti, V. \& Galiazzo, G. (1986). Z. Kristallogr. 177, 315318.

Wang, X. Q., Xia, C. L., Chen, S. B., Tan, J. H., Ou, T. M., Huang, S. L., Li, D., Gu, L. Q. \& Huang, Z. S. (2015). Eur. J. Med. Chem. 89, 349361.

Wood, P. A., Allen, F. H. \& Pidcock, E. (2009). CrystEngComm, 11, 1563-1571.

Xia, H., Liu, Y., Zhao, P., Gou, S. \& Wang, J. (2016). Org. Lett. 18, 1796-1799. 


\section{supporting information}

Acta Cryst. (2020). C76, 883-890 [https://doi.org/10.1107/S2053229620010803]

\section{4-Styrylquinolines from cyclocondensation reactions between (2-aminophenyl)-} chalcones and 1,3-diketones: crystal structures and regiochemistry

\section{Diego Rodríguez, Sergio Andrés Guerrero, Alirio Palma, Justo Cobo and Christopher}

\section{Glidewell}

\section{Computing details}

For all structures, data collection: APEX3 (Bruker, 2018); cell refinement: SAINT (Bruker, 2017); data reduction: SAINT (Bruker, 2017); program(s) used to solve structure: SHELXT2014 (Sheldrick, 2015a); program(s) used to refine structure: SHELXL2014 (Sheldrick, 2015b); molecular graphics: PLATON (Spek, 2020); software used to prepare material for publication: SHELXL2014 (Sheldrick, 2015b) and PLATON (Spek, 2020).

(E)-3-Acetyl-4-[2-(4-methoxyphenyl)ethenyl]-2-methylquinoline (I)

\section{Crystal data}

$\mathrm{C}_{21} \mathrm{H}_{19} \mathrm{NO}_{2}$

$M_{r}=317.37$

Monoclinic, $P 2_{1} / c$

$a=8.2595(4) \AA$

$b=6.4279(3) \AA$

$c=31.9064(14) \AA$

$\beta=93.674(2)^{\circ}$

$V=1690.47(14) \AA^{3}$

$Z=4$

\section{Data collection}

Bruker D8 Venture diffractometer

Radiation source: INCOATEC high brilliance microfocus sealed tube

Multilayer mirror monochromator $\varphi$ and $\omega$ scans

Absorption correction: multi-scan

(SADABS; Bruker, 2016)

$T_{\min }=0.947, T_{\max }=0.993$

Refinement

Refinement on $F^{2}$

Least-squares matrix: full

$R\left[F^{2}>2 \sigma\left(F^{2}\right)\right]=0.040$

$w R\left(F^{2}\right)=0.111$

$S=1.06$

4197 reflections

220 parameters
$F(000)=672$

$D_{\mathrm{x}}=1.247 \mathrm{Mg} \mathrm{m}^{-3}$

Mo $K \alpha$ radiation, $\lambda=0.71073 \AA$

Cell parameters from 4198 reflections

$\theta=2.5-28.3^{\circ}$

$\mu=0.08 \mathrm{~mm}^{-1}$

$T=100 \mathrm{~K}$

Plate, yellow

$0.23 \times 0.16 \times 0.09 \mathrm{~mm}$

39800 measured reflections

4197 independent reflections

3528 reflections with $I>2 \sigma(I)$

$R_{\text {int }}=0.051$

$\theta_{\text {max }}=28.3^{\circ}, \theta_{\min }=2.6^{\circ}$

$h=-11 \rightarrow 11$

$k=-8 \rightarrow 8$

$l=-42 \rightarrow 40$

0 restraints

Primary atom site location: difference Fourier map

Hydrogen site location: inferred from neighbouring sites

$\mathrm{H}$-atom parameters constrained 
$w=1 /\left[\sigma^{2}\left(F_{\mathrm{o}}^{2}\right)+(0.0488 P)^{2}+0.6947 P\right]$

where $P=\left(F_{\mathrm{o}}^{2}+2 F_{\mathrm{c}}{ }^{2}\right) / 3$

$(\Delta / \sigma)_{\max }<0.001$

$$
\Delta \rho_{\max }=0.33 \text { e } \AA^{-3}
$$

$\Delta \rho_{\min }=-0.23$ e $\AA^{-3}$

Special details

Geometry. All esds (except the esd in the dihedral angle between two 1.s. planes) are estimated using the full covariance matrix. The cell esds are taken into account individually in the estimation of esds in distances, angles and torsion angles; correlations between esds in cell parameters are only used when they are defined by crystal symmetry. An approximate (isotropic) treatment of cell esds is used for estimating esds involving 1.s. planes.

Fractional atomic coordinates and isotropic or equivalent isotropic displacement parameters $\left(\AA^{2}\right)$

\begin{tabular}{|c|c|c|c|c|}
\hline & $x$ & $y$ & $z$ & $U_{\text {iso }} * / U_{\text {eq }}$ \\
\hline N1 & $0.22600(12)$ & $0.50352(15)$ & $0.46800(3)$ & $0.0187(2)$ \\
\hline $\mathrm{C} 2$ & $0.35344(13)$ & $0.40197(17)$ & $0.45582(3)$ & $0.0183(2)$ \\
\hline $\mathrm{C} 3$ & $0.44411(13)$ & $0.47008(17)$ & $0.42158(3)$ & $0.0170(2)$ \\
\hline $\mathrm{C} 4$ & $0.39728(13)$ & $0.64671(17)$ & $0.39940(3)$ & $0.0167(2)$ \\
\hline $\mathrm{C} 4 \mathrm{~A}$ & $0.25816(13)$ & $0.75745(17)$ & $0.41187(3)$ & $0.0170(2)$ \\
\hline $\mathrm{C} 5$ & $0.19665(15)$ & $0.93792(19)$ & $0.39100(4)$ & $0.0220(2)$ \\
\hline H5 & 0.2493 & 0.9915 & 0.3677 & $0.026^{*}$ \\
\hline C6 & $0.06173(16)$ & $1.0358(2)$ & $0.40417(4)$ & $0.0256(3)$ \\
\hline H6 & 0.0204 & 1.1553 & 0.3896 & $0.031 *$ \\
\hline $\mathrm{C} 7$ & $-0.01623(15)$ & $0.9608(2)$ & 0.43914 (4) & $0.0247(3)$ \\
\hline $\mathrm{H} 7$ & -0.1088 & 1.0313 & 0.4482 & $0.030^{*}$ \\
\hline $\mathrm{C} 8$ & $0.04033(14)$ & $0.78703(19)$ & $0.46010(4)$ & $0.0209(2)$ \\
\hline H8 & -0.0127 & 0.7380 & 0.4838 & $0.025^{*}$ \\
\hline C8A & $0.17774(13)$ & $0.67992(17)$ & $0.44663(3)$ & $0.0171(2)$ \\
\hline $\mathrm{C} 21$ & 0.40445 (16) & $0.21170(19)$ & $0.48070(4)$ & $0.0238(2)$ \\
\hline $\mathrm{H} 21 \mathrm{~A}$ & 0.3370 & 0.1977 & 0.5047 & $0.036^{*}$ \\
\hline $\mathrm{H} 21 \mathrm{~B}$ & 0.5185 & 0.2251 & 0.4908 & $0.036^{*}$ \\
\hline $\mathrm{H} 21 \mathrm{C}$ & 0.3911 & 0.0883 & 0.4628 & $0.036^{*}$ \\
\hline C31 & $0.59731(14)$ & $0.35584(18)$ & $0.41244(3)$ & $0.0203(2)$ \\
\hline $\mathrm{O} 31$ & $0.59378(12)$ & $0.17673(14)$ & $0.40028(3)$ & $0.0326(2)$ \\
\hline $\mathrm{C} 32$ & $0.75319(14)$ & $0.4693(2)$ & $0.42241(4)$ & $0.0245(3)$ \\
\hline $\mathrm{H} 32 \mathrm{~A}$ & 0.7811 & 0.4627 & 0.4527 & $0.037^{*}$ \\
\hline H32B & 0.7409 & 0.6150 & 0.4137 & $0.037 *$ \\
\hline $\mathrm{H} 32 \mathrm{C}$ & 0.8397 & 0.4045 & 0.4073 & $0.037^{*}$ \\
\hline $\mathrm{C} 41$ & 0.48647 (13) & $0.72134(18)$ & $0.36375(3)$ & $0.0185(2)$ \\
\hline $\mathrm{H} 41$ & 0.5124 & 0.8651 & 0.3625 & $0.022 *$ \\
\hline $\mathrm{C} 42$ & $0.53271(13)$ & $0.59664(18)$ & $0.33313(3)$ & $0.0179(2)$ \\
\hline $\mathrm{H} 42$ & 0.5019 & 0.4545 & 0.3345 & $0.021 *$ \\
\hline $\mathrm{C} 421$ & $0.62670(13)$ & $0.65888(18)$ & $0.29759(3)$ & $0.0176(2)$ \\
\hline $\mathrm{C} 422$ & $0.70887(14)$ & $0.84777(18)$ & $0.29572(3)$ & $0.0202(2)$ \\
\hline $\mathrm{H} 422$ & 0.7091 & 0.9402 & 0.3190 & $0.024^{*}$ \\
\hline $\mathrm{C} 423$ & 0.79067 (14) & $0.90422(19)$ & $0.26062(4)$ & $0.0211(2)$ \\
\hline $\mathrm{H} 423$ & 0.8458 & 1.0338 & 0.2599 & $0.025^{*}$ \\
\hline $\mathrm{C} 424$ & $0.79091(14)$ & $0.76872(19)$ & $0.22647(3)$ & $0.0206(2)$ \\
\hline $\mathrm{C} 425$ & $0.71394(15)$ & $0.57636(19)$ & $0.22835(4)$ & $0.0226(2)$ \\
\hline $\mathrm{H} 425$ & 0.7164 & 0.4823 & 0.2054 & $0.027^{*}$ \\
\hline
\end{tabular}


supporting information

\begin{tabular}{lllll} 
C426 & $0.63392(14)$ & $0.52220(19)$ & $0.26355(3)$ & $0.0200(2)$ \\
H426 & 0.5830 & 0.3900 & 0.2647 & $0.024^{*}$ \\
O424 & $0.86368(11)$ & $0.80822(15)$ & $0.18996(3)$ & $0.0275(2)$ \\
C427 & $0.93382(16)$ & $1.0093(2)$ & $0.18527(4)$ & $0.0274(3)$ \\
H47A & 0.8511 & 1.1163 & 0.1886 & $0.041^{*}$ \\
H47B & 0.9754 & 1.0211 & 0.1573 & $0.041^{*}$ \\
H47C & 1.0231 & 1.0286 & 0.2067 & $0.041^{*}$ \\
\hline
\end{tabular}

Atomic displacement parameters $\left(\AA^{2}\right)$

\begin{tabular}{lllllll}
\hline & $U^{11}$ & $U^{22}$ & $U^{33}$ & $U^{12}$ & $U^{13}$ & $U^{23}$ \\
\hline $\mathrm{N} 1$ & $0.0202(5)$ & $0.0183(5)$ & $0.0177(4)$ & $-0.0008(4)$ & $0.0010(3)$ & $0.0008(4)$ \\
$\mathrm{C} 2$ & $0.0204(5)$ & $0.0159(5)$ & $0.0182(5)$ & $-0.0009(4)$ & $-0.0008(4)$ & $0.0005(4)$ \\
$\mathrm{C} 3$ & $0.0172(5)$ & $0.0161(5)$ & $0.0178(5)$ & $0.0008(4)$ & $0.0006(4)$ & $-0.0020(4)$ \\
$\mathrm{C} 4$ & $0.0185(5)$ & $0.0163(5)$ & $0.0154(5)$ & $0.0009(4)$ & $0.0019(4)$ & $-0.0019(4)$ \\
$\mathrm{C} 4 \mathrm{~A}$ & $0.0190(5)$ & $0.0171(5)$ & $0.0150(5)$ & $0.0015(4)$ & $0.0014(4)$ & $-0.0015(4)$ \\
$\mathrm{C} 5$ & $0.0280(6)$ & $0.0223(6)$ & $0.0162(5)$ & $0.0056(5)$ & $0.0057(4)$ & $0.0025(4)$ \\
$\mathrm{C} 6$ & $0.0292(6)$ & $0.0251(6)$ & $0.0231(6)$ & $0.0098(5)$ & $0.0044(5)$ & $0.0044(5)$ \\
$\mathrm{C} 7$ & $0.0219(6)$ & $0.0271(6)$ & $0.0257(6)$ & $0.0075(5)$ & $0.0059(4)$ & $0.0000(5)$ \\
C8 & $0.0194(5)$ & $0.0246(6)$ & $0.0192(5)$ & $0.0002(4)$ & $0.0044(4)$ & $-0.0002(4)$ \\
C8A & $0.0176(5)$ & $0.0178(5)$ & $0.0157(5)$ & $-0.0005(4)$ & $0.0006(4)$ & $-0.0011(4)$ \\
C21 & $0.0295(6)$ & $0.0185(5)$ & $0.0234(6)$ & $0.0028(5)$ & $0.0013(5)$ & $0.0048(4)$ \\
C31 & $0.0237(6)$ & $0.0188(5)$ & $0.0185(5)$ & $0.0046(4)$ & $0.0016(4)$ & $0.0004(4)$ \\
O31 & $0.0335(5)$ & $0.0205(4)$ & $0.0437(6)$ & $0.0063(4)$ & $0.0013(4)$ & $-0.0083(4)$ \\
C32 & $0.0204(6)$ & $0.0273(6)$ & $0.0259(6)$ & $0.0038(5)$ & $0.0030(4)$ & $-0.0002(5)$ \\
C41 & $0.0201(5)$ & $0.0176(5)$ & $0.0180(5)$ & $0.0019(4)$ & $0.0030(4)$ & $0.0013(4)$ \\
C42 & $0.0176(5)$ & $0.0182(5)$ & $0.0178(5)$ & $0.0009(4)$ & $0.0009(4)$ & $-0.0001(4)$ \\
C421 & $0.0166(5)$ & $0.0197(5)$ & $0.0164(5)$ & $0.0025(4)$ & $0.0015(4)$ & $-0.0011(4)$ \\
C422 & $0.0205(5)$ & $0.0215(6)$ & $0.0188(5)$ & $0.0011(4)$ & $0.0030(4)$ & $-0.0044(4)$ \\
C423 & $0.0205(5)$ & $0.0211(5)$ & $0.0220(5)$ & $-0.0023(4)$ & $0.0032(4)$ & $-0.0025(4)$ \\
C424 & $0.0194(5)$ & $0.0256(6)$ & $0.0173(5)$ & $0.0007(4)$ & $0.0042(4)$ & $-0.0003(4)$ \\
C425 & $0.0264(6)$ & $0.0244(6)$ & $0.0173(5)$ & $-0.0020(5)$ & $0.0043(4)$ & $-0.0055(4)$ \\
C426 & $0.0206(5)$ & $0.0205(5)$ & $0.0189(5)$ & $-0.0010(4)$ & $0.0018(4)$ & $-0.0029(4)$ \\
O424 & $0.0341(5)$ & $0.0297(5)$ & $0.0199(4)$ & $-0.0070(4)$ & $0.0109(3)$ & $-0.0027(3)$ \\
C427 & $0.0276(6)$ & $0.0314(7)$ & $0.0240(6)$ & $-0.0065(5)$ & $0.0066(5)$ & $0.0027(5)$ \\
& & & & & & \\
\hline & & & & & &
\end{tabular}

Geometric parameters $\left(\stackrel{A}{A}{ }^{\circ}\right)$

\begin{tabular}{llll}
\hline $\mathrm{N} 1-\mathrm{C} 2$ & $1.3181(15)$ & $\mathrm{C} 32-\mathrm{H} 32 \mathrm{~A}$ & 0.9800 \\
$\mathrm{~N} 1-\mathrm{C} 8 \mathrm{~A}$ & $1.3692(14)$ & $\mathrm{C} 32-\mathrm{H} 32 \mathrm{~B}$ & 0.9800 \\
$\mathrm{C} 2-\mathrm{C} 3$ & $1.4319(15)$ & $\mathrm{C} 32-\mathrm{H} 32 \mathrm{C}$ & 0.9800 \\
$\mathrm{C} 2-\mathrm{C} 21$ & $1.5036(16)$ & $\mathrm{C} 41-\mathrm{C} 42$ & $1.3382(15)$ \\
$\mathrm{C} 3-\mathrm{C} 4$ & $1.3800(15)$ & $\mathrm{C} 41-\mathrm{H} 41$ & 0.9500 \\
$\mathrm{C} 3-\mathrm{C} 31$ & $1.5076(15)$ & $\mathrm{C} 42-\mathrm{C} 421$ & $1.4701(15)$ \\
$\mathrm{C} 4-\mathrm{C} 4 \mathrm{~A}$ & $1.4296(15)$ & $\mathrm{C} 42-\mathrm{H} 42$ & 0.9500 \\
$\mathrm{C} 4-\mathrm{C} 41$ & $1.4746(15)$ & $\mathrm{C} 421-\mathrm{C} 422$ & $1.3941(16)$ \\
$\mathrm{C} 4 \mathrm{~A}-\mathrm{C} 5$ & $1.4157(16)$ & $\mathrm{C} 421-\mathrm{C} 426$ & $1.4012(15)$ \\
$\mathrm{C} 4 \mathrm{~A}-\mathrm{C} 8 \mathrm{~A}$ & $1.4190(15)$ & $\mathrm{C} 422-\mathrm{C} 423$ & $1.3926(16)$
\end{tabular}




\begin{tabular}{|c|c|c|c|}
\hline $\mathrm{C} 5-\mathrm{C} 6$ & $1.3691(16)$ & $\mathrm{C} 422-\mathrm{H} 422$ & 0.9500 \\
\hline $\mathrm{C} 5-\mathrm{H} 5$ & 0.9500 & $\mathrm{C} 423-\mathrm{C} 424$ & $1.3952(16)$ \\
\hline $\mathrm{C} 6-\mathrm{C} 7$ & $1.4087(17)$ & $\mathrm{C} 423-\mathrm{H} 423$ & 0.9500 \\
\hline $\mathrm{C} 6-\mathrm{H} 6$ & 0.9500 & $\mathrm{C} 424-\mathrm{O} 424$ & $1.3684(13)$ \\
\hline $\mathrm{C} 7-\mathrm{C} 8$ & $1.3685(17)$ & $\mathrm{C} 424-\mathrm{C} 425$ & $1.3934(17)$ \\
\hline $\mathrm{C} 7-\mathrm{H} 7$ & 0.9500 & $\mathrm{C} 425-\mathrm{C} 426$ & $1.3837(15)$ \\
\hline $\mathrm{C} 8-\mathrm{C} 8 \mathrm{~A}$ & $1.4176(15)$ & $\mathrm{C} 425-\mathrm{H} 425$ & 0.9500 \\
\hline $\mathrm{C} 8-\mathrm{H} 8$ & 0.9500 & $\mathrm{C} 426-\mathrm{H} 426$ & 0.9500 \\
\hline $\mathrm{C} 21-\mathrm{H} 21 \mathrm{~A}$ & 0.9800 & $\mathrm{O} 424-\mathrm{C} 427$ & $1.4282(15)$ \\
\hline $\mathrm{C} 21-\mathrm{H} 21 \mathrm{~B}$ & 0.9800 & $\mathrm{C} 427-\mathrm{H} 47 \mathrm{~A}$ & 0.9800 \\
\hline $\mathrm{C} 21-\mathrm{H} 21 \mathrm{C}$ & 0.9800 & $\mathrm{C} 427-\mathrm{H} 47 \mathrm{~B}$ & 0.9800 \\
\hline $\mathrm{C} 31-\mathrm{O} 31$ & $1.2147(15)$ & $\mathrm{C} 427-\mathrm{H} 47 \mathrm{C}$ & 0.9800 \\
\hline $\mathrm{C} 31-\mathrm{C} 32$ & $1.4959(17)$ & & \\
\hline $\mathrm{C} 2-\mathrm{N} 1-\mathrm{C} 8 \mathrm{~A}$ & $118.41(9)$ & $\mathrm{C} 31-\mathrm{C} 32-\mathrm{H} 32 \mathrm{~A}$ & 109.5 \\
\hline $\mathrm{N} 1-\mathrm{C} 2-\mathrm{C} 3$ & $122.69(10)$ & С $31-\mathrm{C} 32-\mathrm{H} 32 \mathrm{~B}$ & 109.5 \\
\hline $\mathrm{N} 1-\mathrm{C} 2-\mathrm{C} 21$ & $116.69(10)$ & $\mathrm{H} 32 \mathrm{~A}-\mathrm{C} 32-\mathrm{H} 32 \mathrm{~B}$ & 109.5 \\
\hline $\mathrm{C} 3-\mathrm{C} 2-\mathrm{C} 21$ & $120.59(10)$ & $\mathrm{C} 31-\mathrm{C} 32-\mathrm{H} 32 \mathrm{C}$ & 109.5 \\
\hline $\mathrm{C} 4-\mathrm{C} 3-\mathrm{C} 2$ & $119.97(10)$ & $\mathrm{H} 32 \mathrm{~A}-\mathrm{C} 32-\mathrm{H} 32 \mathrm{C}$ & 109.5 \\
\hline $\mathrm{C} 4-\mathrm{C} 3-\mathrm{C} 31$ & $120.84(10)$ & $\mathrm{H} 32 \mathrm{~B}-\mathrm{C} 32-\mathrm{H} 32 \mathrm{C}$ & 109.5 \\
\hline $\mathrm{C} 2-\mathrm{C} 3-\mathrm{C} 31$ & $119.03(10)$ & $\mathrm{C} 42-\mathrm{C} 41-\mathrm{C} 4$ & $123.26(11)$ \\
\hline $\mathrm{C} 3-\mathrm{C} 4-\mathrm{C} 4 \mathrm{~A}$ & $118.09(10)$ & $\mathrm{C} 42-\mathrm{C} 41-\mathrm{H} 41$ & 118.4 \\
\hline $\mathrm{C} 3-\mathrm{C} 4-\mathrm{C} 41$ & $121.72(10)$ & $\mathrm{C} 4-\mathrm{C} 41-\mathrm{H} 41$ & 118.4 \\
\hline $\mathrm{C} 4 \mathrm{~A}-\mathrm{C} 4-\mathrm{C} 41$ & $120.19(10)$ & $\mathrm{C} 41-\mathrm{C} 42-\mathrm{C} 421$ & $126.13(11)$ \\
\hline $\mathrm{C} 5-\mathrm{C} 4 \mathrm{~A}-\mathrm{C} 8 \mathrm{~A}$ & $118.92(10)$ & $\mathrm{C} 41-\mathrm{C} 42-\mathrm{H} 42$ & 116.9 \\
\hline $\mathrm{C} 5-\mathrm{C} 4 \mathrm{~A}-\mathrm{C} 4$ & $123.21(10)$ & $\mathrm{C} 421-\mathrm{C} 42-\mathrm{H} 42$ & 116.9 \\
\hline $\mathrm{C} 8 \mathrm{~A}-\mathrm{C} 4 \mathrm{~A}-\mathrm{C} 4$ & $117.87(10)$ & $\mathrm{C} 422-\mathrm{C} 421-\mathrm{C} 426$ & $117.90(10)$ \\
\hline $\mathrm{C} 6-\mathrm{C} 5-\mathrm{C} 4 \mathrm{~A}$ & $120.45(11)$ & $\mathrm{C} 422-\mathrm{C} 421-\mathrm{C} 42$ & $123.54(10)$ \\
\hline $\mathrm{C} 6-\mathrm{C} 5-\mathrm{H} 5$ & 119.8 & $\mathrm{C} 426-\mathrm{C} 421-\mathrm{C} 42$ & $118.56(10)$ \\
\hline $\mathrm{C} 4 \mathrm{~A}-\mathrm{C} 5-\mathrm{H} 5$ & 119.8 & $\mathrm{C} 423-\mathrm{C} 422-\mathrm{C} 421$ & $121.59(10)$ \\
\hline $\mathrm{C} 5-\mathrm{C} 6-\mathrm{C} 7$ & $120.54(11)$ & $\mathrm{C} 423-\mathrm{C} 422-\mathrm{H} 422$ & 119.2 \\
\hline $\mathrm{C} 5-\mathrm{C} 6-\mathrm{H} 6$ & 119.7 & $\mathrm{C} 421-\mathrm{C} 422-\mathrm{H} 422$ & 119.2 \\
\hline $\mathrm{C} 7-\mathrm{C} 6-\mathrm{H} 6$ & 119.7 & $\mathrm{C} 422-\mathrm{C} 423-\mathrm{C} 424$ & $119.38(11)$ \\
\hline $\mathrm{C} 8-\mathrm{C} 7-\mathrm{C} 6$ & $120.49(11)$ & $\mathrm{C} 422-\mathrm{C} 423-\mathrm{H} 423$ & 120.3 \\
\hline $\mathrm{C} 8-\mathrm{C} 7-\mathrm{H} 7$ & 119.8 & $\mathrm{C} 424-\mathrm{C} 423-\mathrm{H} 423$ & 120.3 \\
\hline $\mathrm{C} 6-\mathrm{C} 7-\mathrm{H} 7$ & 119.8 & $\mathrm{O} 424-\mathrm{C} 424-\mathrm{C} 425$ & $115.33(10)$ \\
\hline $\mathrm{C} 7-\mathrm{C} 8-\mathrm{C} 8 \mathrm{~A}$ & $120.20(10)$ & $\mathrm{O} 424-\mathrm{C} 424-\mathrm{C} 423$ & $124.88(11)$ \\
\hline $\mathrm{C} 7-\mathrm{C} 8-\mathrm{H} 8$ & 119.9 & $\mathrm{C} 425-\mathrm{C} 424-\mathrm{C} 423$ & $119.79(10)$ \\
\hline $\mathrm{C} 8 \mathrm{~A}-\mathrm{C} 8-\mathrm{H} 8$ & 119.9 & $\mathrm{C} 426-\mathrm{C} 425-\mathrm{C} 424$ & $120.08(11)$ \\
\hline $\mathrm{N} 1-\mathrm{C} 8 \mathrm{~A}-\mathrm{C} 8$ & $117.67(10)$ & $\mathrm{C} 426-\mathrm{C} 425-\mathrm{H} 425$ & 120.0 \\
\hline $\mathrm{N} 1-\mathrm{C} 8 \mathrm{~A}-\mathrm{C} 4 \mathrm{~A}$ & $122.96(10)$ & $\mathrm{C} 424-\mathrm{C} 425-\mathrm{H} 425$ & 120.0 \\
\hline $\mathrm{C} 8-\mathrm{C} 8 \mathrm{~A}-\mathrm{C} 4 \mathrm{~A}$ & $119.37(10)$ & $\mathrm{C} 425-\mathrm{C} 426-\mathrm{C} 421$ & $121.18(11)$ \\
\hline $\mathrm{C} 2-\mathrm{C} 21-\mathrm{H} 21 \mathrm{~A}$ & 109.5 & $\mathrm{C} 425-\mathrm{C} 426-\mathrm{H} 426$ & 119.4 \\
\hline $\mathrm{C} 2-\mathrm{C} 21-\mathrm{H} 21 \mathrm{~B}$ & 109.5 & $\mathrm{C} 421-\mathrm{C} 426-\mathrm{H} 426$ & 119.4 \\
\hline $\mathrm{H} 21 \mathrm{~A}-\mathrm{C} 21-\mathrm{H} 21 \mathrm{~B}$ & 109.5 & $\mathrm{C} 424-\mathrm{O} 424-\mathrm{C} 427$ & $117.40(9)$ \\
\hline $\mathrm{C} 2-\mathrm{C} 21-\mathrm{H} 21 \mathrm{C}$ & 109.5 & $\mathrm{O} 424-\mathrm{C} 427-\mathrm{H} 47 \mathrm{~A}$ & 109.5 \\
\hline $\mathrm{H} 21 \mathrm{~A}-\mathrm{C} 21-\mathrm{H} 21 \mathrm{C}$ & 109.5 & $\mathrm{O} 424-\mathrm{C} 427-\mathrm{H} 47 \mathrm{~B}$ & 109.5 \\
\hline $\mathrm{H} 21 \mathrm{~B}-\mathrm{C} 21-\mathrm{H} 21 \mathrm{C}$ & 109.5 & $\mathrm{H} 47 \mathrm{~A}-\mathrm{C} 427-\mathrm{H} 47 \mathrm{~B}$ & 109.5 \\
\hline
\end{tabular}




$\begin{array}{ll}\mathrm{O} 31-\mathrm{C} 31-\mathrm{C} 32 & 122.18(11) \\ \mathrm{O} 31-\mathrm{C} 31-\mathrm{C} 3 & 121.35(11) \\ \mathrm{C} 32-\mathrm{C} 31-\mathrm{C} 3 & 116.30(10) \\ & \\ \mathrm{C} 8 \mathrm{~A}-\mathrm{N} 1-\mathrm{C} 2-\mathrm{C} 3 & 0.77(16) \\ \mathrm{C} 8 \mathrm{~A}-\mathrm{N} 1-\mathrm{C} 2-\mathrm{C} 21 & 178.74(10) \\ \mathrm{N} 1-\mathrm{C} 2-\mathrm{C} 3-\mathrm{C} 4 & -1.17(17) \\ \mathrm{C} 21-\mathrm{C} 2-\mathrm{C} 3-\mathrm{C} 4 & -179.06(10) \\ \mathrm{N} 1-\mathrm{C} 2-\mathrm{C} 3-\mathrm{C} 31 & 174.26(10) \\ \mathrm{C} 21-\mathrm{C} 2-\mathrm{C} 3-\mathrm{C} 31 & -3.63(16) \\ \mathrm{C} 2-\mathrm{C} 3-\mathrm{C} 4-\mathrm{C} 4 \mathrm{~A} & 0.39(16) \\ \mathrm{C} 31-\mathrm{C} 3-\mathrm{C} 4-\mathrm{C} 4 \mathrm{~A} & -174.96(10) \\ \mathrm{C} 2-\mathrm{C} 3-\mathrm{C} 4-\mathrm{C} 41 & -179.53(10) \\ \mathrm{C} 31-\mathrm{C} 3-\mathrm{C} 4-\mathrm{C} 41 & 5.12(16) \\ \mathrm{C} 3-\mathrm{C} 4-\mathrm{C} 4 \mathrm{~A}-\mathrm{C} 5 & -178.64(11) \\ \mathrm{C} 41-\mathrm{C} 4-\mathrm{C} 4 \mathrm{~A}-\mathrm{C} 5 & 1.29(17) \\ \mathrm{C} 3-\mathrm{C} 4-\mathrm{C} 4 \mathrm{~A}-\mathrm{C} 8 \mathrm{~A} & 0.65(15) \\ \mathrm{C} 41-\mathrm{C} 4-\mathrm{C} 4 \mathrm{~A}-\mathrm{C} 8 \mathrm{~A} & -179.43(10) \\ \mathrm{C} 8 \mathrm{~A}-\mathrm{C} 4 \mathrm{~A}-\mathrm{C} 5-\mathrm{C} 6 & -0.01(18) \\ \mathrm{C} 4-\mathrm{C} 4 \mathrm{~A}-\mathrm{C} 5-\mathrm{C} 6 & 179.27(12) \\ \mathrm{C} 4 \mathrm{~A}-\mathrm{C} 5-\mathrm{C} 6-\mathrm{C} 7 & 1.2(2) \\ \mathrm{C} 5-\mathrm{C} 6-\mathrm{C} 7-\mathrm{C} 8 & -1.0(2) \\ \mathrm{C} 6-\mathrm{C} 7-\mathrm{C} 8-\mathrm{C} 8 \mathrm{~A} & -0.36(19) \\ \mathrm{C} 2-\mathrm{N} 1-\mathrm{C} 8 \mathrm{~A}-\mathrm{C} 8 & 179.91(10) \\ \mathrm{C} 2-\mathrm{N} 1-\mathrm{C} 8 \mathrm{~A}-\mathrm{C} 4 \mathrm{~A} & 0.36(16) \\ \mathrm{C} 7-\mathrm{C} 8-\mathrm{C} 8 \mathrm{~A}-\mathrm{N} 1 & -178.08(11) \\ \mathrm{C} 7-\mathrm{C} 8-\mathrm{C} 8 \mathrm{~A}-\mathrm{C} 4 \mathrm{~A} & 1.49(17) \\ \mathrm{C} 5-\mathrm{C} 4 \mathrm{~A}-\mathrm{C} 8 \mathrm{~A}-\mathrm{N} 1 & 178.24(10) \\ & \end{array}$

$\begin{array}{ll}\mathrm{O} 424-\mathrm{C} 427-\mathrm{H} 47 \mathrm{C} & 109.5 \\ \mathrm{H} 47 \mathrm{~A}-\mathrm{C} 427-\mathrm{H} 47 \mathrm{C} & 109.5 \\ \mathrm{H} 47 \mathrm{~B}-\mathrm{C} 427-\mathrm{H} 47 \mathrm{C} & 109.5 \\ & \\ \mathrm{C} 4-\mathrm{C} 4 \mathrm{~A}-\mathrm{C} 8 \mathrm{~A}-\mathrm{N} 1 & -1.07(16) \\ \mathrm{C} 5-\mathrm{C} 4 \mathrm{~A}-\mathrm{C} 8 \mathrm{~A}-\mathrm{C} 8 & -1.30(16) \\ \mathrm{C} 4-\mathrm{C} 4 \mathrm{~A}-\mathrm{C} 8 \mathrm{~A}-\mathrm{C} 8 & 179.38(10) \\ \mathrm{C} 4-\mathrm{C} 3-\mathrm{C} 31-\mathrm{O} 31 & -118.43(13) \\ \mathrm{C} 2-\mathrm{C} 3-\mathrm{C} 31-\mathrm{O} 31 & 66.18(15) \\ \mathrm{C} 4-\mathrm{C} 3-\mathrm{C} 31-\mathrm{C} 32 & 66.26(14) \\ \mathrm{C} 2-\mathrm{C} 3-\mathrm{C} 31-\mathrm{C} 32 & -109.13(12) \\ \mathrm{C} 3-\mathrm{C} 4-\mathrm{C} 41-\mathrm{C} 42 & 46.40(16) \\ \mathrm{C} 4 \mathrm{~A}-\mathrm{C} 4-\mathrm{C} 41-\mathrm{C} 42 & -133.52(12) \\ \mathrm{C} 4-\mathrm{C} 41-\mathrm{C} 42-\mathrm{C} 421 & -177.66(10) \\ \mathrm{C} 41-\mathrm{C} 42-\mathrm{C} 421-\mathrm{C} 422 & 13.78(18) \\ \mathrm{C} 41-\mathrm{C} 42-\mathrm{C} 421-\mathrm{C} 426 & -165.87(11) \\ \mathrm{C} 426-\mathrm{C} 421-\mathrm{C} 422-\mathrm{C} 423 & 2.48(17) \\ \mathrm{C} 42-\mathrm{C} 421-\mathrm{C} 422-\mathrm{C} 423 & -177.17(11) \\ \mathrm{C} 421-\mathrm{C} 422-\mathrm{C} 423-\mathrm{C} 424 & -0.12(18) \\ \mathrm{C} 422-\mathrm{C} 423-\mathrm{C} 424-\mathrm{O} 424 & 178.54(11) \\ \mathrm{C} 422-\mathrm{C} 423-\mathrm{C} 424-\mathrm{C} 425 & -1.99(18) \\ \mathrm{O} 424-\mathrm{C} 424-\mathrm{C} 425-\mathrm{C} 426 & -178.81(11) \\ \mathrm{C} 423-\mathrm{C} 424-\mathrm{C} 425-\mathrm{C} 426 & 1.68(18) \\ \mathrm{C} 424-\mathrm{C} 425-\mathrm{C} 426-\mathrm{C} 421 & 0.77(18) \\ \mathrm{C} 422-\mathrm{C} 421-\mathrm{C} 426-\mathrm{C} 425 & -2.80(17) \\ \mathrm{C} 42-\mathrm{C} 421-\mathrm{C} 426-\mathrm{C} 425 & 176.87(11) \\ \mathrm{C} 425-\mathrm{C} 424-\mathrm{O} 424-\mathrm{C} 427 & 175.49(11) \\ \mathrm{C} 423-\mathrm{C} 424-\mathrm{O} 424-\mathrm{C} 427 & -5.02(17) \\ & \end{array}$

Hydrogen-bond geometry $\left(A,{ }^{\circ}\right)$

\begin{tabular}{lllll}
\hline$D-\mathrm{H}^{\cdots} \cdots A$ & $D-\mathrm{H}$ & $\mathrm{H} \cdots A$ & $D \cdots A$ & $D-\mathrm{H} \cdots A$ \\
\hline $\mathrm{C} 41-\mathrm{H} 41 \cdots \mathrm{O} 31^{\mathrm{i}}$ & 0.95 & 2.41 & $3.2527(15)$ & 148 \\
$\mathrm{C} 426-\mathrm{H} 426 \cdots C g 3^{\mathrm{ii}}$ & 0.95 & 2.77 & $3.5252(13)$ & 138 \\
\hline
\end{tabular}

Symmetry codes: (i) $x, y+1, z$; (ii) $-x+1, y-1 / 2,-z+1 / 2$.

(E)-3-Acetyl-4-[2-(4-bromophenyl)ethenyl]-2-methylquinoline (II)

Crystal data

$\mathrm{C}_{20} \mathrm{H}_{16} \mathrm{BrNO}$

$M_{r}=366.24$

Monoclinic, $P 2_{1} / c$

$a=8.0849(3) \AA$

$b=6.6692(2) \AA$

$c=31.1063(10) \AA$

$\beta=95.005(1)^{\circ}$

$V=1670.85(10) \AA^{3}$

$Z=4$
$F(000)=744$

$D_{\mathrm{x}}=1.456 \mathrm{Mg} \mathrm{m}^{-3}$

Mo $K \alpha$ radiation, $\lambda=0.71073 \AA$

Cell parameters from 3839 reflections

$\theta=2.5-27.5^{\circ}$

$\mu=2.46 \mathrm{~mm}^{-1}$

$T=100 \mathrm{~K}$

Plate, yellow

$0.17 \times 0.11 \times 0.04 \mathrm{~mm}$ 


\section{Data collection}

Bruker D8 Venture diffractometer

Radiation source: INCOATEC high brilliance microfocus sealed tube

Multilayer mirror monochromator $\varphi$ and $\omega$ scans

Absorption correction: multi-scan

(SADABS; Bruker, 2016)

$T_{\min }=0.810, T_{\max }=0.906$

\section{Refinement}

Refinement on $F^{2}$

Least-squares matrix: full

$R\left[F^{2}>2 \sigma\left(F^{2}\right)\right]=0.030$

$w R\left(F^{2}\right)=0.073$

$S=1.03$

3839 reflections

210 parameters

0 restraints
38342 measured reflections

3839 independent reflections

3434 reflections with $I>2 \sigma(I)$

$R_{\text {int }}=0.034$

$\theta_{\max }=27.5^{\circ}, \theta_{\min }=2.5^{\circ}$

$h=-10 \rightarrow 10$

$k=-8 \rightarrow 8$

$l=-39 \rightarrow 40$
Primary atom site location: difference Fourier map

Hydrogen site location: inferred from neighbouring sites

$\mathrm{H}$-atom parameters constrained

$w=1 /\left[\sigma^{2}\left(F_{\mathrm{o}}^{2}\right)+(0.0294 P)^{2}+1.5332 P\right]$ where $P=\left(F_{\mathrm{o}}{ }^{2}+2 F_{\mathrm{c}}{ }^{2}\right) / 3$

$(\Delta / \sigma)_{\max }=0.001$

$\Delta \rho_{\max }=0.56 \mathrm{e} \AA^{-3}$

$\Delta \rho_{\min }=-0.76$ e $\AA^{-3}$

Special details

Geometry. All esds (except the esd in the dihedral angle between two 1.s. planes) are estimated using the full covariance matrix. The cell esds are taken into account individually in the estimation of esds in distances, angles and torsion angles; correlations between esds in cell parameters are only used when they are defined by crystal symmetry. An approximate (isotropic) treatment of cell esds is used for estimating esds involving 1.s. planes.

Fractional atomic coordinates and isotropic or equivalent isotropic displacement parameters $\left(\AA^{2}\right)$

\begin{tabular}{lllll}
\hline & $x$ & $y$ & $z$ & $U_{\text {iso }} * / U_{\text {eq }}$ \\
\hline N1 & $0.22878(18)$ & $0.4980(2)$ & $0.46764(5)$ & $0.0216(3)$ \\
C2 3 & $0.3566(2)$ & $0.4009(2)$ & $0.45402(5)$ & $0.0214(3)$ \\
C4 & $0.4453(2)$ & $0.4719(2)$ & $0.41918(5)$ & $0.0198(3)$ \\
C4A & $0.3970(2)$ & $0.6464(2)$ & $0.39805(5)$ & $0.0198(3)$ \\
C5 & $0.2553(2)$ & $0.7499(2)$ & $0.41163(5)$ & $0.0211(3)$ \\
H5 & $0.1897(2)$ & $0.9251(3)$ & $0.39104(6)$ & $0.0314(4)$ \\
C6 & 0.2403 & 0.9788 & 0.3671 & $0.038^{*}$ \\
H6 & $0.0535(3)$ & $1.0184(3)$ & $0.40534(7)$ & $0.0358(5)$ \\
C7 & 0.0093 & 1.1347 & 0.3909 & $0.043^{*}$ \\
H7 & $-0.0209(2)$ & $0.9429(3)$ & $0.44112(6)$ & $0.0302(4)$ \\
C8 & -0.1138 & 1.0101 & 0.4511 & $0.036^{*}$ \\
H8 & $0.0395(2)$ & $0.7731(3)$ & $0.46174(6)$ & $0.0249(3)$ \\
C8A & -0.0110 & 0.7237 & 0.4861 & $0.030^{*}$ \\
C21 & $0.1770(2)$ & $0.6713(2)$ & $0.44684(5)$ & $0.0203(3)$ \\
H21A & $0.4107(2)$ & $0.2140(3)$ & $0.47846(6)$ & $0.0290(4)$ \\
H21B & 0.3457 & 0.1989 & 0.5034 & $0.043^{*}$ \\
H21C & 0.5288 & 0.2242 & 0.4884 & $0.043^{*}$ \\
C31 & 0.3927 & 0.0972 & 0.4595 & $0.043^{*}$ \\
& $0.5978(2)$ & $0.3597(3)$ & $0.40803(6)$ & $0.0248(3)$
\end{tabular}




$\begin{array}{lllll}\mathrm{O} 31 & 0.58723(19) & 0.1923(2) & 0.39267(5) & 0.0402(3) \\ \mathrm{C} 32 & 0.7612(2) & 0.4597(3) & 0.41940(7) & 0.0315(4) \\ \mathrm{H} 32 \mathrm{~A} & 0.7915 & 0.4466 & 0.4505 & 0.047^{*} \\ \mathrm{H} 32 \mathrm{~B} & 0.7527 & 0.6021 & 0.4117 & 0.047^{*} \\ \mathrm{H} 32 \mathrm{C} & 0.8465 & 0.3961 & 0.4035 & 0.047^{*} \\ \mathrm{C} 41 & 0.4876(2) & 0.7319(3) & 0.36336(5) & 0.0221(3) \\ \mathrm{H} 41 & 0.5115 & 0.8713 & 0.3646 & 0.026^{*} \\ \mathrm{C} 42 & 0.5388(2) & 0.6286(3) & 0.33029(5) & 0.0218(3) \\ \mathrm{H} 42 & 0.5101 & 0.4906 & 0.3282 & 0.026^{*} \\ \mathrm{C} 421 & 0.63654(19) & 0.7121(3) & 0.29676(5) & 0.0223(3) \\ \mathrm{C} 422 & 0.7176(2) & 0.8969(3) & 0.30115(6) & 0.0263(4) \\ \mathrm{H} 422 & 0.7123 & 0.9718 & 0.3270 & 0.032^{*} \\ \mathrm{C} 423 & 0.8056(2) & 0.9731(3) & 0.26847(6) & 0.0298(4) \\ \mathrm{H} 423 & 0.8603 & 1.0989 & 0.2717 & 0.036^{*} \\ \mathrm{C} 424 & 0.8123(2) & 0.8621(3) & 0.23090(6) & 0.0303(4) \\ \mathrm{B} 42 & 0.93043(2) & 0.96822(4) & 0.18577(2) & 0.04244(9) \\ \mathrm{C} 425 & 0.7361(2) & 0.6784(4) & 0.22581(6) & 0.0368(5) \\ \mathrm{H} 425 & 0.7429 & 0.6037 & 0.2000 & 0.044^{*} \\ \mathrm{C} 426 & 0.6488(2) & 0.6033(3) & 0.25888(6) & 0.0304(4) \\ \mathrm{H} 426 & 0.5967 & 0.4758 & 0.2556 & 0.037^{*} \\ & & & & \end{array}$

Atomic displacement parameters $\left(\AA^{2}\right)$

\begin{tabular}{lllllll}
\hline & $U^{11}$ & $U^{22}$ & $U^{33}$ & $U^{12}$ & $U^{13}$ & $U^{23}$ \\
\hline $\mathrm{N} 1$ & $0.0235(7)$ & $0.0197(7)$ & $0.0218(7)$ & $-0.0007(5)$ & $0.0040(5)$ & $0.0022(5)$ \\
$\mathrm{C} 2$ & $0.0252(8)$ & $0.0163(7)$ & $0.0228(8)$ & $-0.0003(6)$ & $0.0019(6)$ & $0.0013(6)$ \\
$\mathrm{C} 3$ & $0.0221(7)$ & $0.0167(7)$ & $0.0209(8)$ & $0.0011(6)$ & $0.0025(6)$ & $-0.0029(6)$ \\
$\mathrm{C} 4$ & $0.0234(7)$ & $0.0181(7)$ & $0.0183(7)$ & $0.0017(6)$ & $0.0035(6)$ & $-0.0014(6)$ \\
$\mathrm{C} 4 \mathrm{~A}$ & $0.0248(8)$ & $0.0206(8)$ & $0.0183(7)$ & $0.0039(6)$ & $0.0037(6)$ & $0.0008(6)$ \\
$\mathrm{C} 5$ & $0.0389(10)$ & $0.0314(10)$ & $0.0257(9)$ & $0.0141(8)$ & $0.0128(7)$ & $0.0104(7)$ \\
$\mathrm{C} 6$ & $0.0420(11)$ & $0.0338(10)$ & $0.0329(10)$ & $0.0195(9)$ & $0.0106(8)$ & $0.0112(8)$ \\
$\mathrm{C} 7$ & $0.0282(9)$ & $0.0318(10)$ & $0.0317(9)$ & $0.0111(7)$ & $0.0095(7)$ & $0.0020(8)$ \\
$\mathrm{C} 8$ & $0.0243(8)$ & $0.0280(9)$ & $0.0231(8)$ & $0.0017(7)$ & $0.0068(6)$ & $0.0004(7)$ \\
$\mathrm{C} 8 \mathrm{~A}$ & $0.0220(7)$ & $0.0196(8)$ & $0.0193(7)$ & $0.0005(6)$ & $0.0024(6)$ & $0.0001(6)$ \\
$\mathrm{C} 21$ & $0.0359(9)$ & $0.0205(8)$ & $0.0312(9)$ & $0.0040(7)$ & $0.0066(7)$ & $0.0076(7)$ \\
$\mathrm{C} 31$ & $0.0309(9)$ & $0.0193(8)$ & $0.0250(8)$ & $0.0067(7)$ & $0.0076(7)$ & $0.0026(6)$ \\
O31 & $0.0482(8)$ & $0.0202(7)$ & $0.0543(9)$ & $0.0054(6)$ & $0.0170(7)$ & $-0.0086(6)$ \\
C32 & $0.0252(9)$ & $0.0346(10)$ & $0.0351(10)$ & $0.0085(7)$ & $0.0041(7)$ & $0.0015(8)$ \\
C41 & $0.0259(8)$ & $0.0195(8)$ & $0.0215(8)$ & $0.0035(6)$ & $0.0058(6)$ & $0.0022(6)$ \\
C42 & $0.0205(7)$ & $0.0239(8)$ & $0.0212(8)$ & $0.0009(6)$ & $0.0025(6)$ & $-0.0013(6)$ \\
C421 & $0.0182(7)$ & $0.0305(9)$ & $0.0184(8)$ & $0.0026(6)$ & $0.0026(6)$ & $-0.0014(6)$ \\
C422 & $0.0247(8)$ & $0.0319(9)$ & $0.0227(8)$ & $0.0003(7)$ & $0.0046(6)$ & $-0.0039(7)$ \\
C423 & $0.0253(8)$ & $0.0352(10)$ & $0.0295(9)$ & $-0.0019(7)$ & $0.0059(7)$ & $0.0019(8)$ \\
C424 & $0.0198(8)$ & $0.0518(12)$ & $0.0200(8)$ & $0.0001(8)$ & $0.0054(6)$ & $0.0042(8)$ \\
Br42 & $0.03101(11)$ & $0.07124(17)$ & $0.02652(11)$ & $-0.00315(9)$ & $0.01070(8)$ & $0.01007(9)$ \\
C425 & $0.0309(9)$ & $0.0599(14)$ & $0.0205(9)$ & $-0.0071(9)$ & $0.0063(7)$ & $-0.0119(9)$ \\
C426 & $0.0267(8)$ & $0.0405(11)$ & $0.0246(9)$ & $-0.0067(8)$ & $0.0053(7)$ & $-0.0100(8)$ \\
& & & & & & \\
\hline
\end{tabular}


Geometric parameters $\left(\AA,{ }^{\circ}\right)$

\begin{tabular}{|c|c|c|c|}
\hline $\mathrm{N} 1-\mathrm{C} 2$ & $1.321(2)$ & $\mathrm{C} 31-\mathrm{O} 31$ & $1.214(2)$ \\
\hline $\mathrm{N} 1-\mathrm{C} 8 \mathrm{~A}$ & $1.372(2)$ & $\mathrm{C} 31-\mathrm{C} 32$ & $1.495(3)$ \\
\hline $\mathrm{C} 2-\mathrm{C} 3$ & $1.431(2)$ & $\mathrm{C} 32-\mathrm{H} 32 \mathrm{~A}$ & 0.9800 \\
\hline $\mathrm{C} 2-\mathrm{C} 21$ & $1.505(2)$ & $\mathrm{C} 32-\mathrm{H} 32 \mathrm{~B}$ & 0.9800 \\
\hline $\mathrm{C} 3-\mathrm{C} 4$ & $1.377(2)$ & $\mathrm{C} 32-\mathrm{H} 32 \mathrm{C}$ & 0.9800 \\
\hline $\mathrm{C} 3-\mathrm{C} 31$ & $1.509(2)$ & $\mathrm{C} 41-\mathrm{C} 42$ & $1.334(2)$ \\
\hline $\mathrm{C} 4-\mathrm{C} 4 \mathrm{~A}$ & $1.432(2)$ & $\mathrm{C} 41-\mathrm{H} 41$ & 0.9500 \\
\hline $\mathrm{C} 4-\mathrm{C} 41$ & $1.471(2)$ & $\mathrm{C} 42-\mathrm{C} 421$ & $1.472(2)$ \\
\hline $\mathrm{C} 4 \mathrm{~A}-\mathrm{C} 8 \mathrm{~A}$ & $1.413(2)$ & $\mathrm{C} 42-\mathrm{H} 42$ & 0.9500 \\
\hline $\mathrm{C} 4 \mathrm{~A}-\mathrm{C} 5$ & $1.413(2)$ & $\mathrm{C} 421-\mathrm{C} 426$ & $1.395(2)$ \\
\hline $\mathrm{C} 5-\mathrm{C} 6$ & $1.372(3)$ & $\mathrm{C} 421-\mathrm{C} 422$ & $1.397(3)$ \\
\hline $\mathrm{C} 5-\mathrm{H} 5$ & 0.9500 & $\mathrm{C} 422-\mathrm{C} 423$ & $1.387(3)$ \\
\hline $\mathrm{C} 6-\mathrm{C} 7$ & $1.404(3)$ & $\mathrm{C} 422-\mathrm{H} 422$ & 0.9500 \\
\hline $\mathrm{C} 6-\mathrm{H} 6$ & 0.9500 & $\mathrm{C} 423-\mathrm{C} 424$ & $1.388(3)$ \\
\hline $\mathrm{C} 7-\mathrm{C} 8$ & $1.371(3)$ & $\mathrm{C} 423-\mathrm{H} 423$ & 0.9500 \\
\hline $\mathrm{C} 7-\mathrm{H} 7$ & 0.9500 & $\mathrm{C} 424-\mathrm{C} 425$ & $1.375(3)$ \\
\hline $\mathrm{C} 8-\mathrm{C} 8 \mathrm{~A}$ & $1.415(2)$ & $\mathrm{C} 424-\mathrm{Br} 42$ & $1.9018(17)$ \\
\hline $\mathrm{C} 8-\mathrm{H} 8$ & 0.9500 & $\mathrm{C} 425-\mathrm{C} 426$ & $1.390(3)$ \\
\hline $\mathrm{C} 21-\mathrm{H} 21 \mathrm{~A}$ & 0.9800 & $\mathrm{C} 425-\mathrm{H} 425$ & 0.9500 \\
\hline $\mathrm{C} 21-\mathrm{H} 21 \mathrm{~B}$ & 0.9800 & $\mathrm{C} 426-\mathrm{H} 426$ & 0.9500 \\
\hline $\mathrm{C} 21-\mathrm{H} 21 \mathrm{C}$ & 0.9800 & & \\
\hline $\mathrm{C} 2-\mathrm{N} 1-\mathrm{C} 8 \mathrm{~A}$ & $118.51(14)$ & $\mathrm{O} 31-\mathrm{C} 31-\mathrm{C} 32$ & $122.29(16)$ \\
\hline $\mathrm{N} 1-\mathrm{C} 2-\mathrm{C} 3$ & $122.57(15)$ & $\mathrm{O} 31-\mathrm{C} 31-\mathrm{C} 3$ & $121.02(17)$ \\
\hline $\mathrm{N} 1-\mathrm{C} 2-\mathrm{C} 21$ & $116.44(15)$ & $\mathrm{C} 32-\mathrm{C} 31-\mathrm{C} 3$ & $116.59(15)$ \\
\hline $\mathrm{C} 3-\mathrm{C} 2-\mathrm{C} 21$ & $120.94(15)$ & $\mathrm{C} 31-\mathrm{C} 32-\mathrm{H} 32 \mathrm{~A}$ & 109.5 \\
\hline $\mathrm{C} 4-\mathrm{C} 3-\mathrm{C} 2$ & $120.10(15)$ & $\mathrm{C} 31-\mathrm{C} 32-\mathrm{H} 32 \mathrm{~B}$ & 109.5 \\
\hline $\mathrm{C} 4-\mathrm{C} 3-\mathrm{C} 31$ & $120.88(15)$ & $\mathrm{H} 32 \mathrm{~A}-\mathrm{C} 32-\mathrm{H} 32 \mathrm{~B}$ & 109.5 \\
\hline $\mathrm{C} 2-\mathrm{C} 3-\mathrm{C} 31$ & $118.92(14)$ & $\mathrm{C} 31-\mathrm{C} 32-\mathrm{H} 32 \mathrm{C}$ & 109.5 \\
\hline $\mathrm{C} 3-\mathrm{C} 4-\mathrm{C} 4 \mathrm{~A}$ & $117.88(15)$ & $\mathrm{H} 32 \mathrm{~A}-\mathrm{C} 32-\mathrm{H} 32 \mathrm{C}$ & 109.5 \\
\hline $\mathrm{C} 3-\mathrm{C} 4-\mathrm{C} 41$ & $122.63(14)$ & $\mathrm{H} 32 \mathrm{~B}-\mathrm{C} 32-\mathrm{H} 32 \mathrm{C}$ & 109.5 \\
\hline $\mathrm{C} 4 \mathrm{~A}-\mathrm{C} 4-\mathrm{C} 41$ & $119.47(14)$ & $\mathrm{C} 42-\mathrm{C} 41-\mathrm{C} 4$ & $125.02(16)$ \\
\hline $\mathrm{C} 8 \mathrm{~A}-\mathrm{C} 4 \mathrm{~A}-\mathrm{C} 5$ & $118.84(15)$ & $\mathrm{C} 42-\mathrm{C} 41-\mathrm{H} 41$ & 117.5 \\
\hline $\mathrm{C} 8 \mathrm{~A}-\mathrm{C} 4 \mathrm{~A}-\mathrm{C} 4$ & $118.27(15)$ & $\mathrm{C} 4-\mathrm{C} 41-\mathrm{H} 41$ & 117.5 \\
\hline $\mathrm{C} 5-\mathrm{C} 4 \mathrm{~A}-\mathrm{C} 4$ & $122.89(15)$ & $\mathrm{C} 41-\mathrm{C} 42-\mathrm{C} 421$ & $124.98(16)$ \\
\hline $\mathrm{C} 6-\mathrm{C} 5-\mathrm{C} 4 \mathrm{~A}$ & $120.53(17)$ & $\mathrm{C} 41-\mathrm{C} 42-\mathrm{H} 42$ & 117.5 \\
\hline C6- $55-\mathrm{H} 5$ & 119.7 & $\mathrm{C} 421-\mathrm{C} 42-\mathrm{H} 42$ & 117.5 \\
\hline $\mathrm{C} 4 \mathrm{~A}-\mathrm{C} 5-\mathrm{H} 5$ & 119.7 & $\mathrm{C} 426-\mathrm{C} 421-\mathrm{C} 422$ & $118.32(16)$ \\
\hline $\mathrm{C} 5-\mathrm{C} 6-\mathrm{C} 7$ & $120.41(17)$ & $\mathrm{C} 426-\mathrm{C} 421-\mathrm{C} 42$ & $118.94(16)$ \\
\hline $\mathrm{C} 5-\mathrm{C} 6-\mathrm{H} 6$ & 119.8 & $\mathrm{C} 422-\mathrm{C} 421-\mathrm{C} 42$ & $122.74(15)$ \\
\hline $\mathrm{C} 7-\mathrm{C} 6-\mathrm{H} 6$ & 119.8 & $\mathrm{C} 423-\mathrm{C} 422-\mathrm{C} 421$ & $121.18(17)$ \\
\hline $\mathrm{C} 8-\mathrm{C} 7-\mathrm{C} 6$ & $120.49(16)$ & $\mathrm{C} 423-\mathrm{C} 422-\mathrm{H} 422$ & 119.4 \\
\hline $\mathrm{C} 8-\mathrm{C} 7-\mathrm{H} 7$ & 119.8 & $\mathrm{C} 421-\mathrm{C} 422-\mathrm{H} 422$ & 119.4 \\
\hline $\mathrm{C} 6-\mathrm{C} 7-\mathrm{H} 7$ & 119.8 & $\mathrm{C} 422-\mathrm{C} 423-\mathrm{C} 424$ & $118.80(18)$ \\
\hline $\mathrm{C} 7-\mathrm{C} 8-\mathrm{C} 8 \mathrm{~A}$ & $120.09(16)$ & $\mathrm{C} 422-\mathrm{C} 423-\mathrm{H} 423$ & 120.6 \\
\hline $\mathrm{C} 7-\mathrm{C} 8-\mathrm{H} 8$ & 120.0 & $\mathrm{C} 424-\mathrm{C} 423-\mathrm{H} 423$ & 120.6 \\
\hline
\end{tabular}




\begin{tabular}{|c|c|}
\hline $\mathrm{C} 8 \mathrm{~A}-\mathrm{C} 8-\mathrm{H} 8$ & 120.0 \\
\hline $\mathrm{N} 1-\mathrm{C} 8 \mathrm{~A}-\mathrm{C} 4 \mathrm{~A}$ & $122.63(15)$ \\
\hline $\mathrm{N} 1-\mathrm{C} 8 \mathrm{~A}-\mathrm{C} 8$ & $117.77(15)$ \\
\hline $\mathrm{C} 4 \mathrm{~A}-\mathrm{C} 8 \mathrm{~A}-\mathrm{C} 8$ & $119.59(15)$ \\
\hline $\mathrm{C} 2-\mathrm{C} 21-\mathrm{H} 21 \mathrm{~A}$ & 109.5 \\
\hline $\mathrm{C} 2-\mathrm{C} 21-\mathrm{H} 21 \mathrm{~B}$ & 109.5 \\
\hline $\mathrm{H} 21 \mathrm{~A}-\mathrm{C} 21-\mathrm{H} 21 \mathrm{~B}$ & 109.5 \\
\hline $\mathrm{C} 2-\mathrm{C} 21-\mathrm{H} 21 \mathrm{C}$ & 109.5 \\
\hline $\mathrm{H} 21 \mathrm{~A}-\mathrm{C} 21-\mathrm{H} 21 \mathrm{C}$ & 109.5 \\
\hline $\mathrm{H} 21 \mathrm{~B}-\mathrm{C} 21-\mathrm{H} 21 \mathrm{C}$ & 109.5 \\
\hline $\mathrm{C} 8 \mathrm{~A}-\mathrm{N} 1-\mathrm{C} 2-\mathrm{C} 3$ & $1.5(2)$ \\
\hline $\mathrm{C} 8 \mathrm{~A}-\mathrm{N} 1-\mathrm{C} 2-\mathrm{C} 21$ & $178.81(15)$ \\
\hline $\mathrm{N} 1-\mathrm{C} 2-\mathrm{C} 3-\mathrm{C} 4$ & $-0.9(3)$ \\
\hline $\mathrm{C} 21-\mathrm{C} 2-\mathrm{C} 3-\mathrm{C} 4$ & $-178.04(16)$ \\
\hline $\mathrm{N} 1-\mathrm{C} 2-\mathrm{C} 3-\mathrm{C} 31$ & $175.33(15)$ \\
\hline $\mathrm{C} 21-\mathrm{C} 2-\mathrm{C} 3-\mathrm{C} 31$ & $-1.8(2)$ \\
\hline $\mathrm{C} 2-\mathrm{C} 3-\mathrm{C} 4-\mathrm{C} 4 \mathrm{~A}$ & $-1.1(2)$ \\
\hline $\mathrm{C} 31-\mathrm{C} 3-\mathrm{C} 4-\mathrm{C} 4 \mathrm{~A}$ & $-177.20(15)$ \\
\hline $\mathrm{C} 2-\mathrm{C} 3-\mathrm{C} 4-\mathrm{C} 41$ & $177.13(15)$ \\
\hline $\mathrm{C} 31-\mathrm{C} 3-\mathrm{C} 4-\mathrm{C} 41$ & $1.0(2)$ \\
\hline $\mathrm{C} 3-\mathrm{C} 4-\mathrm{C} 4 \mathrm{~A}-\mathrm{C} 8 \mathrm{~A}$ & $2.2(2)$ \\
\hline $\mathrm{C} 41-\mathrm{C} 4-\mathrm{C} 4 \mathrm{~A}-\mathrm{C} 8 \mathrm{~A}$ & $-176.01(15)$ \\
\hline $\mathrm{C} 3-\mathrm{C} 4-\mathrm{C} 4 \mathrm{~A}-\mathrm{C} 5$ & $-176.96(17)$ \\
\hline $\mathrm{C} 41-\mathrm{C} 4-\mathrm{C} 4 \mathrm{~A}-\mathrm{C} 5$ & $4.8(3)$ \\
\hline $\mathrm{C} 8 \mathrm{~A}-\mathrm{C} 4 \mathrm{~A}-\mathrm{C} 5-\mathrm{C} 6$ & $0.7(3)$ \\
\hline $\mathrm{C} 4-\mathrm{C} 4 \mathrm{~A}-\mathrm{C} 5-\mathrm{C} 6$ & $179.93(19)$ \\
\hline $\mathrm{C} 4 \mathrm{~A}-\mathrm{C} 5-\mathrm{C} 6-\mathrm{C} 7$ & $1.1(3)$ \\
\hline $\mathrm{C} 5-\mathrm{C} 6-\mathrm{C} 7-\mathrm{C} 8$ & $-1.2(3)$ \\
\hline $\mathrm{C} 6-\mathrm{C} 7-\mathrm{C} 8-\mathrm{C} 8 \mathrm{~A}$ & $-0.5(3)$ \\
\hline $\mathrm{C} 2-\mathrm{N} 1-\mathrm{C} 8 \mathrm{~A}-\mathrm{C} 4 \mathrm{~A}$ & $-0.2(2)$ \\
\hline $\mathrm{C} 2-\mathrm{N} 1-\mathrm{C} 8 \mathrm{~A}-\mathrm{C} 8$ & $179.77(15)$ \\
\hline $\mathrm{C} 5-\mathrm{C} 4 \mathrm{~A}-\mathrm{C} 8 \mathrm{~A}-\mathrm{N} 1$ & $177.56(16)$ \\
\hline $\mathrm{C} 4-\mathrm{C} 4 \mathrm{~A}-\mathrm{C} 8 \mathrm{~A}-\mathrm{N} 1$ & $-1.7(2)$ \\
\hline
\end{tabular}

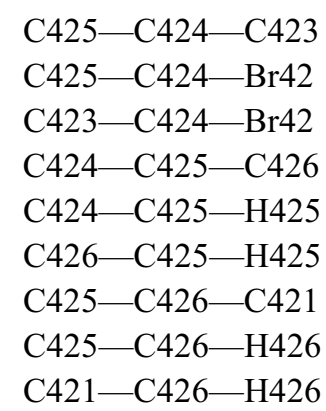

$\mathrm{C} 5-\mathrm{C} 4 \mathrm{~A}-\mathrm{C} 8 \mathrm{~A}-\mathrm{C} 8$

$\mathrm{C} 4-\mathrm{C} 4 \mathrm{~A}-\mathrm{C} 8 \mathrm{~A}-\mathrm{C} 8$

$\mathrm{C} 7-\mathrm{C} 8-\mathrm{C} 8 \mathrm{~A}-\mathrm{N} 1$

$\mathrm{C} 7-\mathrm{C} 8-\mathrm{C} 8 \mathrm{~A}-\mathrm{C} 4 \mathrm{~A}$

$\mathrm{C} 4-\mathrm{C} 3-\mathrm{C} 31-\mathrm{O} 31$

$\mathrm{C} 2-\mathrm{C} 3-\mathrm{C} 31-\mathrm{O} 31$

$\mathrm{C} 4-\mathrm{C} 3-\mathrm{C} 31-\mathrm{C} 32$

$\mathrm{C} 2-\mathrm{C} 3-\mathrm{C} 31-\mathrm{C} 32$

$\mathrm{C} 3-\mathrm{C} 4-\mathrm{C} 41-\mathrm{C} 42$

$\mathrm{C} 4 \mathrm{~A}-\mathrm{C} 4-\mathrm{C} 41-\mathrm{C} 42$

$\mathrm{C} 4-\mathrm{C} 41-\mathrm{C} 42-\mathrm{C} 421$

$\mathrm{C} 41-\mathrm{C} 42-\mathrm{C} 421-\mathrm{C} 426$

$\mathrm{C} 41-\mathrm{C} 42-\mathrm{C} 421-\mathrm{C} 422$

$\mathrm{C} 426-\mathrm{C} 421-\mathrm{C} 422-\mathrm{C} 423$

$\mathrm{C} 42-\mathrm{C} 421-\mathrm{C} 422-\mathrm{C} 423$

$\mathrm{C} 421-\mathrm{C} 422-\mathrm{C} 423-\mathrm{C} 424$

$\mathrm{C} 422-\mathrm{C} 423-\mathrm{C} 424-\mathrm{C} 425$

$\mathrm{C} 422-\mathrm{C} 423-\mathrm{C} 424-\mathrm{Br} 42$

$\mathrm{C} 423-\mathrm{C} 424-\mathrm{C} 425-\mathrm{C} 426$

$\mathrm{Br} 42-\mathrm{C} 424-\mathrm{C} 425-\mathrm{C} 426$

$\mathrm{C} 424-\mathrm{C} 425-\mathrm{C} 426-\mathrm{C} 421$

$\mathrm{C} 422-\mathrm{C} 421-\mathrm{C} 426-\mathrm{C} 425$

$\mathrm{C} 42-\mathrm{C} 421-\mathrm{C} 426-\mathrm{C} 425$
$121.48(17)$

$119.71(14)$

$118.81(15)$

$119.16(17)$

120.4

120.4

121.04 (18)

119.5

119.5

$-2.4(2)$

$178.33(15)$

$-177.65(16)$

$2.3(3)$

$-115.6(2)$

$68.2(2)$

$68.1(2)$

-108.09 (18)

$46.8(3)$

$-135.02(18)$

$-176.86(15)$

$-165.04(17)$

$14.8(3)$

$1.2(3)$

$-178.57(16)$

$0.0(3)$

$-1.0(3)$

$179.13(14)$

0.7 (3)

$-179.40(15)$

$0.6(3)$

$-1.5(3)$

$178.30(17)$

Hydrogen-bond geometry $\left(\AA,{ }^{\circ}\right)$

\begin{tabular}{lllll}
\hline$D-\mathrm{H} \cdots A$ & $D-\mathrm{H}$ & $\mathrm{H} \cdots A$ & $D \cdots A$ & $D-\mathrm{H} \cdots A$ \\
\hline $\mathrm{C} 41-\mathrm{H} 41 \cdots \mathrm{O} 31^{\mathrm{i}}$ & 0.95 & 2.37 & $3.283(2)$ & 161 \\
$\mathrm{C} 426-\mathrm{H} 426 \cdots C g 3^{\mathrm{ii}}$ & 0.95 & 2.91 & $3.7071(19)$ & 142 \\
\hline
\end{tabular}

Symmetry codes: (i) $x, y+1, z$; (ii) $-x+1, y-1 / 2,-z+1 / 2$.

(E)-3-Acetyl-2-methyl-4-\{2-[4-(trifluoromethyl) phenyl] ethenyl\}quinoline (III)

Crystal data

$\mathrm{C}_{21} \mathrm{H}_{16} \mathrm{~F}_{3} \mathrm{NO}$

$M_{r}=355.35$

Monoclinic, $P 2_{1} / c$

$$
\begin{aligned}
& a=8.0822(4) \AA \\
& b=6.6567(4) \AA \\
& c=32.1024(17) \AA
\end{aligned}
$$


$\beta=90.576(2)^{\circ}$

$V=1727.05(16) \AA^{3}$

$Z=4$

$F(000)=736$

$D_{\mathrm{x}}=1.367 \mathrm{Mg} \mathrm{m}^{-3}$

Mo $K \alpha$ radiation, $\lambda=0.71073 \AA$

Data collection

Bruker D8 Venture diffractometer

Radiation source: INCOATEC high brilliance microfocus sealed tube

Multilayer mirror monochromator

$\varphi$ and $\omega$ scans

Absorption correction: multi-scan

(SADABS; Bruker, 2016)

$T_{\min }=0.942, T_{\max }=0.994$

Refinement

Refinement on $F^{2}$

Least-squares matrix: full

$R\left[F^{2}>2 \sigma\left(F^{2}\right)\right]=0.041$

$w R\left(F^{2}\right)=0.101$

$S=1.04$

3959 reflections

237 parameters

0 restraints
Cell parameters from 3961 reflections

$\theta=2.5-27.5^{\circ}$

$\mu=0.11 \mathrm{~mm}^{-1}$

$T=100 \mathrm{~K}$

Needle, colourless

$0.20 \times 0.08 \times 0.06 \mathrm{~mm}$

19648 measured reflections

3959 independent reflections

3363 reflections with $I>2 \sigma(I)$

$R_{\text {int }}=0.032$

$\theta_{\max }=27.5^{\circ}, \theta_{\min }=2.5^{\circ}$

$h=-10 \rightarrow 10$

$k=-8 \rightarrow 8$

$l=-41 \rightarrow 40$

Primary atom site location: difference Fourier map

Hydrogen site location: inferred from neighbouring sites

$\mathrm{H}$-atom parameters constrained

$w=1 /\left[\sigma^{2}\left(F_{\mathrm{o}}^{2}\right)+(0.039 P)^{2}+0.8719 P\right]$ where $P=\left(F_{\mathrm{o}}^{2}+2 F_{\mathrm{c}}^{2}\right) / 3$

$(\Delta / \sigma)_{\max }<0.001$

$\Delta \rho_{\max }=0.28$ e $\AA^{-3}$

$\Delta \rho_{\min }=-0.23$ e $\AA^{-3}$

Special details

Geometry. All esds (except the esd in the dihedral angle between two 1.s. planes) are estimated using the full covariance matrix. The cell esds are taken into account individually in the estimation of esds in distances, angles and torsion angles; correlations between esds in cell parameters are only used when they are defined by crystal symmetry. An approximate (isotropic) treatment of cell esds is used for estimating esds involving l.s. planes.

Fractional atomic coordinates and isotropic or equivalent isotropic displacement parameters $\left(\AA^{2}\right)$

\begin{tabular}{lllll}
\hline & $x$ & $y$ & $z$ & $U_{\text {iso }} * / U_{\text {eq }}$ \\
\hline N1 & $0.24277(14)$ & $0.49703(17)$ & $0.46953(3)$ & $0.0222(2)$ \\
C2 & $0.37353(17)$ & $0.40329(19)$ & $0.45511(4)$ & $0.0214(3)$ \\
C3 & $0.46663(16)$ & $0.47574(19)$ & $0.42048(4)$ & $0.0198(3)$ \\
C4 & $0.41905(16)$ & $0.65014(19)$ & $0.40063(4)$ & $0.0200(3)$ \\
C4A & $0.27453(17)$ & $0.7513(2)$ & $0.41515(4)$ & $0.0213(3)$ \\
C5 & $0.2095(2)$ & $0.9254(2)$ & $0.39603(5)$ & $0.0312(3)$ \\
H5 & 0.2639 & 0.9819 & 0.3727 & $0.037^{*}$ \\
C6 & $0.0690(2)$ & $1.0136(3)$ & $0.41073(5)$ & $0.0382(4)$ \\
H6 & 0.0251 & 1.1291 & 0.3972 & $0.046^{*}$ \\
C7 & $-0.01102(19)$ & $0.9344(2)$ & $0.44580(5)$ & $0.0334(3)$ \\
H7 & -0.1075 & 0.9982 & 0.4561 & $0.040^{*}$ \\
C8 & $0.04868(17)$ & $0.7666(2)$ & $0.46520(4)$ & $0.0263(3)$ \\
H8 & -0.0056 & 0.7150 & 0.4890 & $0.032^{*}$
\end{tabular}




$\begin{array}{lllll}\text { C8A } & 0.19136(16) & 0.6693(2) & 0.44989(4) & 0.0207(3) \\ \text { C21 } & 0.42652(19) & 0.2158(2) & 0.47786(5) & 0.0287(3) \\ \text { H21A } & 0.3609 & 0.2007 & 0.5032 & 0.043^{*} \\ \text { H21B } & 0.5440 & 0.2258 & 0.4854 & 0.043^{*} \\ \text { H21C } & 0.4091 & 0.0988 & 0.4598 & 0.043^{*} \\ \text { C31 } & 0.62124(18) & 0.3660(2) & 0.40773(4) & 0.0248(3) \\ \text { O31 } & 0.61447(15) & 0.20068(16) & 0.39209(4) & 0.0390(3) \\ \text { C32 } & 0.78127(18) & 0.4677(2) & 0.41812(5) & 0.0320(3) \\ \text { H32A } & 0.8021 & 0.4591 & 0.4482 & 0.048^{*} \\ \text { H32B } & 0.7752 & 0.6092 & 0.4098 & 0.048^{*} \\ \text { H32C } & 0.8714 & 0.4016 & 0.4032 & 0.048^{*} \\ \text { C41 } & 0.50967(16) & 0.7346(2) & 0.36505(4) & 0.0218(3) \\ \text { H41 } & 0.5375 & 0.8732 & 0.3659 & 0.026^{*} \\ \text { C42 } & 0.55522(16) & 0.6296(2) & 0.33171(4) & 0.0207(3) \\ \text { H42 } & 0.5279 & 0.4908 & 0.3311 & 0.025^{*} \\ \text { C421 } & 0.64460(15) & 0.7119(2) & 0.29577(4) & 0.0203(3) \\ \text { C422 } & 0.72004(17) & 0.9002(2) & 0.29629(4) & 0.0245(3) \\ \text { H422 } & 0.7169 & 0.9796 & 0.3209 & 0.029^{*} \\ \text { C423 } & 0.79950(17) & 0.9730(2) & 0.26140(4) & 0.0258(3) \\ \text { H423 } & 0.8496 & 1.1021 & 0.2621 & 0.031^{*} \\ \text { C424 } & 0.80597(16) & 0.8576(2) & 0.22548(4) & 0.0236(3) \\ \text { C425 } & 0.73412(18) & 0.6685(2) & 0.22450(4) & 0.0280(3) \\ \text { H425 } & 0.7395 & 0.5887 & 0.2000 & 0.034^{*} \\ \text { C426 } & 0.65429(17) & 0.5967(2) & 0.25951(4) & 0.0255(3) \\ \text { H426 } & 0.6054 & 0.4669 & 0.2588 & 0.031^{*} \\ \text { C427 } & 0.89461(18) & 0.9343(2) & 0.18789(4) & 0.0284(3) \\ \text { F471 } & 1.04888(11) & 0.85971(18) & 0.18484(3) & 0.0461(3) \\ \text { F472 } & 0.90958(14) & 1.13364(15) & 0.18753(3) & 0.0461(3) \\ \text { F473 } & 0.81762(11) & 0.88303(14) & 0.15231(2) & 0.0328(2)\end{array}$

Atomic displacement parameters $\left(\AA^{2}\right)$

\begin{tabular}{lllllll}
\hline & $U^{11}$ & $U^{22}$ & $U^{33}$ & $U^{12}$ & $U^{13}$ & $U^{23}$ \\
\hline N1 & $0.0276(6)$ & $0.0200(6)$ & $0.0190(5)$ & $-0.0029(4)$ & $0.0008(4)$ & $0.0013(4)$ \\
C2 & $0.0292(7)$ & $0.0162(6)$ & $0.0187(6)$ & $-0.0027(5)$ & $-0.0015(5)$ & $0.0002(5)$ \\
C3 & $0.0263(6)$ & $0.0155(6)$ & $0.0177(6)$ & $-0.0003(5)$ & $0.0004(5)$ & $-0.0025(5)$ \\
C4 & $0.0265(6)$ & $0.0176(6)$ & $0.0161(6)$ & $0.0001(5)$ & $0.0010(5)$ & $-0.0021(5)$ \\
C4A & $0.0284(7)$ & $0.0190(6)$ & $0.0165(6)$ & $0.0021(5)$ & $0.0020(5)$ & $-0.0003(5)$ \\
C5 & $0.0418(8)$ & $0.0288(8)$ & $0.0234(7)$ & $0.0117(6)$ & $0.0097(6)$ & $0.0084(6)$ \\
C6 & $0.0478(9)$ & $0.0338(9)$ & $0.0331(8)$ & $0.0201(7)$ & $0.0104(7)$ & $0.0105(7)$ \\
C7 & $0.0337(8)$ & $0.0350(8)$ & $0.0315(8)$ & $0.0112(7)$ & $0.0099(6)$ & $0.0015(7)$ \\
C8 & $0.0280(7)$ & $0.0284(7)$ & $0.0226(7)$ & $0.0001(6)$ & $0.0058(5)$ & $0.0013(6)$ \\
C8A & $0.0253(6)$ & $0.0202(6)$ & $0.0166(6)$ & $-0.0006(5)$ & $0.0001(5)$ & $-0.0006(5)$ \\
C21 & $0.0404(8)$ & $0.0206(7)$ & $0.0250(7)$ & $0.0020(6)$ & $0.0012(6)$ & $0.0056(6)$ \\
C31 & $0.0356(7)$ & $0.0189(7)$ & $0.0199(6)$ & $0.0066(6)$ & $0.0038(5)$ & $0.0025(5)$ \\
O31 & $0.0541(7)$ & $0.0205(5)$ & $0.0426(7)$ & $0.0076(5)$ & $0.0088(5)$ & $-0.0074(5)$ \\
C32 & $0.0281(7)$ & $0.0367(8)$ & $0.0311(8)$ & $0.0088(6)$ & $0.0020(6)$ & $0.0001(7)$ \\
C41 & $0.0267(6)$ & $0.0177(6)$ & $0.0210(6)$ & $0.0024(5)$ & $0.0029(5)$ & $0.0013(5)$
\end{tabular}


supporting information

\begin{tabular}{lllllll} 
C42 & $0.0225(6)$ & $0.0198(6)$ & $0.0200(6)$ & $-0.0003(5)$ & $0.0007(5)$ & $0.0004(5)$ \\
C421 & $0.0191(6)$ & $0.0234(7)$ & $0.0185(6)$ & $0.0024(5)$ & $0.0006(5)$ & $-0.0004(5)$ \\
C422 & $0.0270(7)$ & $0.0262(7)$ & $0.0202(7)$ & $-0.0020(5)$ & $0.0031(5)$ & $-0.0047(5)$ \\
C423 & $0.0265(7)$ & $0.0253(7)$ & $0.0256(7)$ & $-0.0042(5)$ & $0.0023(5)$ & $-0.0005(6)$ \\
C424 & $0.0215(6)$ & $0.0302(7)$ & $0.0193(6)$ & $-0.0008(5)$ & $0.0015(5)$ & $0.0024(6)$ \\
C425 & $0.0333(7)$ & $0.0326(8)$ & $0.0182(7)$ & $-0.0035(6)$ & $0.0038(5)$ & $-0.0052(6)$ \\
C426 & $0.0293(7)$ & $0.0250(7)$ & $0.0224(7)$ & $-0.0039(6)$ & $0.0033(5)$ & $-0.0031(6)$ \\
C427 & $0.0276(7)$ & $0.0349(8)$ & $0.0226(7)$ & $-0.0008(6)$ & $0.0027(5)$ & $0.0038(6)$ \\
F471 & $0.0250(4)$ & $0.0760(8)$ & $0.0377(5)$ & $0.0060(5)$ & $0.0088(4)$ & $0.0219(5)$ \\
F472 & $0.0679(7)$ & $0.0365(5)$ & $0.0341(5)$ & $-0.0151(5)$ & $0.0131(5)$ & $0.0051(4)$ \\
F473 & $0.0361(5)$ & $0.0443(5)$ & $0.0182(4)$ & $0.0004(4)$ & $0.0018(3)$ & $0.0027(4)$ \\
\hline
\end{tabular}

Geometric parameters $\left(\AA,{ }^{\circ}\right)$

\begin{tabular}{|c|c|c|c|}
\hline $\mathrm{N} 1-\mathrm{C} 2$ & $1.3154(18)$ & $\mathrm{C} 31-\mathrm{C} 32$ & $1.495(2)$ \\
\hline $\mathrm{N} 1-\mathrm{C} 8 \mathrm{~A}$ & $1.3710(17)$ & $\mathrm{C} 32-\mathrm{H} 32 \mathrm{~A}$ & 0.9800 \\
\hline $\mathrm{C} 2-\mathrm{C} 3$ & $1.4324(18)$ & $\mathrm{C} 32-\mathrm{H} 32 \mathrm{~B}$ & 0.9800 \\
\hline $\mathrm{C} 2-\mathrm{C} 21$ & $1.5062(18)$ & $\mathrm{C} 32-\mathrm{H} 32 \mathrm{C}$ & 0.9800 \\
\hline $\mathrm{C} 3-\mathrm{C} 4$ & $1.3772(18)$ & $\mathrm{C} 41-\mathrm{C} 42$ & $1.3335(18)$ \\
\hline $\mathrm{C} 3-\mathrm{C} 31$ & $1.5077(18)$ & $\mathrm{C} 41-\mathrm{H} 41$ & 0.9500 \\
\hline $\mathrm{C} 4-\mathrm{C} 4 \mathrm{~A}$ & $1.4304(18)$ & $\mathrm{C} 42-\mathrm{C} 421$ & $1.4729(18)$ \\
\hline $\mathrm{C} 4-\mathrm{C} 41$ & $1.4746(18)$ & $\mathrm{C} 42-\mathrm{H} 42$ & 0.9500 \\
\hline $\mathrm{C} 4 \mathrm{~A}-\mathrm{C} 5$ & $1.4102(19)$ & $\mathrm{C} 421-\mathrm{C} 422$ & $1.3942(19)$ \\
\hline $\mathrm{C} 4 \mathrm{~A}-\mathrm{C} 8 \mathrm{~A}$ & $1.4175(18)$ & $\mathrm{C} 421-\mathrm{C} 426$ & $1.3969(19)$ \\
\hline $\mathrm{C} 5-\mathrm{C} 6$ & $1.367(2)$ & $\mathrm{C} 422-\mathrm{C} 423$ & $1.3843(19)$ \\
\hline $\mathrm{C} 5-\mathrm{H} 5$ & 0.9500 & $\mathrm{C} 422-\mathrm{H} 422$ & 0.9500 \\
\hline $\mathrm{C} 6-\mathrm{C} 7$ & $1.407(2)$ & $\mathrm{C} 423-\mathrm{C} 424$ & $1.387(2)$ \\
\hline C6-H6 & 0.9500 & $\mathrm{C} 423-\mathrm{H} 423$ & 0.9500 \\
\hline $\mathrm{C} 7-\mathrm{C} 8$ & $1.365(2)$ & $\mathrm{C} 424-\mathrm{C} 425$ & $1.386(2)$ \\
\hline $\mathrm{C} 7-\mathrm{H} 7$ & 0.9500 & $\mathrm{C} 424-\mathrm{C} 427$ & $1.4991(19)$ \\
\hline $\mathrm{C} 8-\mathrm{C} 8 \mathrm{~A}$ & $1.4151(19)$ & $\mathrm{C} 425-\mathrm{C} 426$ & $1.3866(19)$ \\
\hline $\mathrm{C} 8-\mathrm{H} 8$ & 0.9500 & $\mathrm{C} 425-\mathrm{H} 425$ & 0.9500 \\
\hline $\mathrm{C} 21-\mathrm{H} 21 \mathrm{~A}$ & 0.9800 & $\mathrm{C} 426-\mathrm{H} 426$ & 0.9500 \\
\hline $\mathrm{C} 21-\mathrm{H} 21 \mathrm{~B}$ & 0.9800 & C427-F472 & $1.3326(18)$ \\
\hline $\mathrm{C} 21-\mathrm{H} 21 \mathrm{C}$ & 0.9800 & C427-F473 & $1.3392(17)$ \\
\hline $\mathrm{C} 31-\mathrm{O} 31$ & $1.2105(18)$ & C427-F471 & $1.3465(17)$ \\
\hline $\mathrm{C} 2-\mathrm{N} 1-\mathrm{C} 8 \mathrm{~A}$ & $118.41(11)$ & $\mathrm{C} 31-\mathrm{C} 32-\mathrm{H} 32 \mathrm{~A}$ & 109.5 \\
\hline $\mathrm{N} 1-\mathrm{C} 2-\mathrm{C} 3$ & $122.93(12)$ & $\mathrm{C} 31-\mathrm{C} 32-\mathrm{H} 32 \mathrm{~B}$ & 109.5 \\
\hline $\mathrm{N} 1-\mathrm{C} 2-\mathrm{C} 21$ & $116.62(12)$ & $\mathrm{H} 32 \mathrm{~A}-\mathrm{C} 32-\mathrm{H} 32 \mathrm{~B}$ & 109.5 \\
\hline $\mathrm{C} 3-\mathrm{C} 2-\mathrm{C} 21$ & $120.42(12)$ & $\mathrm{C} 31-\mathrm{C} 32-\mathrm{H} 32 \mathrm{C}$ & 109.5 \\
\hline $\mathrm{C} 4-\mathrm{C} 3-\mathrm{C} 2$ & $119.77(12)$ & $\mathrm{H} 32 \mathrm{~A}-\mathrm{C} 32-\mathrm{H} 32 \mathrm{C}$ & 109.5 \\
\hline $\mathrm{C} 4-\mathrm{C} 3-\mathrm{C} 31$ & $120.76(12)$ & $\mathrm{H} 32 \mathrm{~B}-\mathrm{C} 32-\mathrm{H} 32 \mathrm{C}$ & 109.5 \\
\hline $\mathrm{C} 2-\mathrm{C} 3-\mathrm{C} 31$ & $119.39(12)$ & $\mathrm{C} 42-\mathrm{C} 41-\mathrm{C} 4$ & $124.41(12)$ \\
\hline $\mathrm{C} 3-\mathrm{C} 4-\mathrm{C} 4 \mathrm{~A}$ & $118.13(12)$ & $\mathrm{C} 42-\mathrm{C} 41-\mathrm{H} 41$ & 117.8 \\
\hline $\mathrm{C} 3-\mathrm{C} 4-\mathrm{C} 41$ & $122.77(12)$ & $\mathrm{C} 4-\mathrm{C} 41-\mathrm{H} 41$ & 117.8 \\
\hline $\mathrm{C} 4 \mathrm{~A}-\mathrm{C} 4-\mathrm{C} 41$ & $119.10(12)$ & $\mathrm{C} 41-\mathrm{C} 42-\mathrm{C} 421$ & $125.11(13)$ \\
\hline $\mathrm{C} 5-\mathrm{C} 4 \mathrm{~A}-\mathrm{C} 8 \mathrm{~A}$ & $118.78(12)$ & $\mathrm{C} 41-\mathrm{C} 42-\mathrm{H} 42$ & 117.4 \\
\hline
\end{tabular}




\begin{tabular}{|c|c|}
\hline $\mathrm{C} 5-\mathrm{C} 4 \mathrm{~A}-\mathrm{C} 4$ & $123.17(12)$ \\
\hline $\mathrm{C} 8 \mathrm{~A}-\mathrm{C} 4 \mathrm{~A}-\mathrm{C} 4$ & $118.04(12)$ \\
\hline $\mathrm{C} 6-\mathrm{C} 5-\mathrm{C} 4 \mathrm{~A}$ & $120.67(14)$ \\
\hline $\mathrm{C} 6-\mathrm{C} 5-\mathrm{H} 5$ & 119.7 \\
\hline $\mathrm{C} 4 \mathrm{~A}-\mathrm{C} 5-\mathrm{H} 5$ & 119.7 \\
\hline $\mathrm{C} 5-\mathrm{C} 6-\mathrm{C} 7$ & $120.36(14)$ \\
\hline $\mathrm{C} 5-\mathrm{C} 6-\mathrm{H} 6$ & 119.8 \\
\hline $\mathrm{C} 7-\mathrm{C} 6-\mathrm{H} 6$ & 119.8 \\
\hline $\mathrm{C} 8-\mathrm{C} 7-\mathrm{C} 6$ & $120.60(14)$ \\
\hline $\mathrm{C} 8-\mathrm{C} 7-\mathrm{H} 7$ & 119.7 \\
\hline $\mathrm{C} 6-\mathrm{C} 7-\mathrm{H} 7$ & 119.7 \\
\hline $\mathrm{C} 7-\mathrm{C} 8-\mathrm{C} 8 \mathrm{~A}$ & $120.11(13)$ \\
\hline $\mathrm{C} 7-\mathrm{C} 8-\mathrm{H} 8$ & 119.9 \\
\hline $\mathrm{C} 8 \mathrm{~A}-\mathrm{C} 8-\mathrm{H} 8$ & 119.9 \\
\hline $\mathrm{N} 1-\mathrm{C} 8 \mathrm{~A}-\mathrm{C} 8$ & $117.86(12)$ \\
\hline $\mathrm{N} 1-\mathrm{C} 8 \mathrm{~A}-\mathrm{C} 4 \mathrm{~A}$ & $122.69(12)$ \\
\hline $\mathrm{C} 8-\mathrm{C} 8 \mathrm{~A}-\mathrm{C} 4 \mathrm{~A}$ & $119.44(12)$ \\
\hline $\mathrm{C} 2-\mathrm{C} 21-\mathrm{H} 21 \mathrm{~A}$ & 109.5 \\
\hline $\mathrm{C} 2-\mathrm{C} 21-\mathrm{H} 21 \mathrm{~B}$ & 109.5 \\
\hline $\mathrm{H} 21 \mathrm{~A}-\mathrm{C} 21-\mathrm{H} 21 \mathrm{~B}$ & 109.5 \\
\hline $\mathrm{C} 2-\mathrm{C} 21-\mathrm{H} 21 \mathrm{C}$ & 109.5 \\
\hline $\mathrm{H} 21 \mathrm{~A}-\mathrm{C} 21-\mathrm{H} 21 \mathrm{C}$ & 109.5 \\
\hline $\mathrm{H} 21 \mathrm{~B}-\mathrm{C} 21-\mathrm{H} 21 \mathrm{C}$ & 109.5 \\
\hline $\mathrm{O} 31-\mathrm{C} 31-\mathrm{C} 32$ & $122.68(14)$ \\
\hline $\mathrm{O} 31-\mathrm{C} 31-\mathrm{C} 3$ & $121.26(14)$ \\
\hline $\mathrm{C} 32-\mathrm{C} 31-\mathrm{C} 3$ & $115.94(12)$ \\
\hline $\mathrm{C} 8 \mathrm{~A}-\mathrm{N} 1-\mathrm{C} 2-\mathrm{C} 3$ & $1.51(19)$ \\
\hline $\mathrm{C} 8 \mathrm{~A}-\mathrm{N} 1-\mathrm{C} 2-\mathrm{C} 21$ & $179.47(12)$ \\
\hline $\mathrm{N} 1-\mathrm{C} 2-\mathrm{C} 3-\mathrm{C} 4$ & $-0.5(2)$ \\
\hline $\mathrm{C} 21-\mathrm{C} 2-\mathrm{C} 3-\mathrm{C} 4$ & $-178.38(12)$ \\
\hline $\mathrm{N} 1-\mathrm{C} 2-\mathrm{C} 3-\mathrm{C} 31$ & $176.10(12)$ \\
\hline $\mathrm{C} 21-\mathrm{C} 2-\mathrm{C} 3-\mathrm{C} 31$ & $-1.78(19)$ \\
\hline $\mathrm{C} 2-\mathrm{C} 3-\mathrm{C} 4-\mathrm{C} 4 \mathrm{~A}$ & $-1.18(19)$ \\
\hline $\mathrm{C} 31-\mathrm{C} 3-\mathrm{C} 4-\mathrm{C} 4 \mathrm{~A}$ & $-177.74(12)$ \\
\hline $\mathrm{C} 2-\mathrm{C} 3-\mathrm{C} 4-\mathrm{C} 41$ & $179.44(12)$ \\
\hline $\mathrm{C} 31-\mathrm{C} 3-\mathrm{C} 4-\mathrm{C} 41$ & $2.9(2)$ \\
\hline $\mathrm{C} 3-\mathrm{C} 4-\mathrm{C} 4 \mathrm{~A}-\mathrm{C} 5$ & $-176.83(13)$ \\
\hline $\mathrm{C} 41-\mathrm{C} 4-\mathrm{C} 4 \mathrm{~A}-\mathrm{C} 5$ & $2.6(2)$ \\
\hline $\mathrm{C} 3-\mathrm{C} 4-\mathrm{C} 4 \mathrm{~A}-\mathrm{C} 8 \mathrm{~A}$ & $1.77(19)$ \\
\hline $\mathrm{C} 41-\mathrm{C} 4-\mathrm{C} 4 \mathrm{~A}-\mathrm{C} 8 \mathrm{~A}$ & $-178.83(12)$ \\
\hline $\mathrm{C} 8 \mathrm{~A}-\mathrm{C} 4 \mathrm{~A}-\mathrm{C} 5-\mathrm{C} 6$ & $0.1(2)$ \\
\hline $\mathrm{C} 4-\mathrm{C} 4 \mathrm{~A}-\mathrm{C} 5-\mathrm{C} 6$ & $178.67(15)$ \\
\hline $\mathrm{C} 4 \mathrm{~A}-\mathrm{C} 5-\mathrm{C} 6-\mathrm{C} 7$ & $1.4(3)$ \\
\hline $\mathrm{C} 5-\mathrm{C} 6-\mathrm{C} 7-\mathrm{C} 8$ & $-1.1(3)$ \\
\hline $\mathrm{C} 6-\mathrm{C} 7-\mathrm{C} 8-\mathrm{C} 8 \mathrm{~A}$ & $-0.6(2)$ \\
\hline $\mathrm{C} 2-\mathrm{N} 1-\mathrm{C} 8 \mathrm{~A}-\mathrm{C} 8$ & $178.81(12)$ \\
\hline $\mathrm{C} 2-\mathrm{N} 1-\mathrm{C} 8 \mathrm{~A}-\mathrm{C} 4 \mathrm{~A}$ & $-0.84(19)$ \\
\hline
\end{tabular}

$$
\begin{aligned}
& \mathrm{C} 421-\mathrm{C} 42-\mathrm{H} 42 \\
& \mathrm{C} 422-\mathrm{C} 421-\mathrm{C} 426 \\
& \mathrm{C} 422-\mathrm{C} 421-\mathrm{C} 42 \\
& \mathrm{C} 426-\mathrm{C} 421-\mathrm{C} 42 \\
& \mathrm{C} 423-\mathrm{C} 422-\mathrm{C} 421 \\
& \mathrm{C} 423-\mathrm{C} 422-\mathrm{H} 422 \\
& \mathrm{C} 421-\mathrm{C} 422-\mathrm{H} 422 \\
& \mathrm{C} 422-\mathrm{C} 423-\mathrm{C} 424 \\
& \mathrm{C} 422-\mathrm{C} 423-\mathrm{H} 423 \\
& \mathrm{C} 424-\mathrm{C} 423-\mathrm{H} 423 \\
& \mathrm{C} 425-\mathrm{C} 424-\mathrm{C} 423 \\
& \mathrm{C} 425-\mathrm{C} 424-\mathrm{C} 427 \\
& \mathrm{C} 423-\mathrm{C} 424-\mathrm{C} 427 \\
& \mathrm{C} 424-\mathrm{C} 425-\mathrm{C} 426 \\
& \text { C424-C425-H425 } \\
& \mathrm{C} 426 \text { - C425-H425 } \\
& \mathrm{C} 425-\mathrm{C} 426-\mathrm{C} 421 \\
& \mathrm{C} 425-\mathrm{C} 426-\mathrm{H} 426 \\
& \mathrm{C} 421-\mathrm{C} 426-\mathrm{H} 426 \\
& \text { F472-C427-F473 } \\
& \text { F472-C427-F471 } \\
& \text { F473-C427-F471 } \\
& \text { F472-C427-C424 } \\
& \text { F473-C427-C424 } \\
& \text { F471-C427-C424 }
\end{aligned}
$$$$
\mathrm{C} 4-\mathrm{C} 4 \mathrm{~A}-\mathrm{C} 8 \mathrm{~A}-\mathrm{C} 8
$$$$
\mathrm{C} 4-\mathrm{C} 3-\mathrm{C} 31-\mathrm{O} 31
$$$$
\mathrm{C} 2-\mathrm{C} 3-\mathrm{C} 31-\mathrm{O} 31
$$$$
\mathrm{C} 4-\mathrm{C} 3-\mathrm{C} 31-\mathrm{C} 32
$$$$
\mathrm{C} 2-\mathrm{C} 3-\mathrm{C} 31-\mathrm{C} 32
$$$$
\mathrm{C} 3-\mathrm{C} 4-\mathrm{C} 41-\mathrm{C} 42
$$$$
\mathrm{C} 4 \mathrm{~A}-\mathrm{C} 4-\mathrm{C} 41-\mathrm{C} 42
$$$$
\mathrm{C} 4-\mathrm{C} 41-\mathrm{C} 42-\mathrm{C} 421
$$$$
\mathrm{C} 41-\mathrm{C} 42-\mathrm{C} 421-\mathrm{C} 422
$$$$
\mathrm{C} 41-\mathrm{C} 42-\mathrm{C} 421-\mathrm{C} 426
$$$$
\mathrm{C} 426-\mathrm{C} 421-\mathrm{C} 422-\mathrm{C} 423
$$$$
\mathrm{C} 42-\mathrm{C} 421-\mathrm{C} 422-\mathrm{C} 423
$$$$
\mathrm{C} 421-\mathrm{C} 422-\mathrm{C} 423-\mathrm{C} 424
$$$$
\mathrm{C} 422-\mathrm{C} 423-\mathrm{C} 424-\mathrm{C} 425
$$$$
\mathrm{C} 422-\mathrm{C} 423-\mathrm{C} 424-\mathrm{C} 427
$$$$
\mathrm{C} 423-\mathrm{C} 424-\mathrm{C} 425-\mathrm{C} 426
$$$$
\mathrm{C} 427-\mathrm{C} 424-\mathrm{C} 425-\mathrm{C} 426
$$$$
\mathrm{C} 424-\mathrm{C} 425-\mathrm{C} 426-\mathrm{C} 421
$$$$
\mathrm{C} 422-\mathrm{C} 421-\mathrm{C} 426-\mathrm{C} 425
$$$$
\mathrm{C} 42-\mathrm{C} 421-\mathrm{C} 426-\mathrm{C} 425
$$$$
\mathrm{C} 425-\mathrm{C} 424-\mathrm{C} 427-\mathrm{F} 472
$$

117.4

$118.39(12)$

$122.90(12)$

$118.72(12)$

$120.76(13)$

119.6

119.6

$120.03(13)$

120.0

120.0

120.15 (13)

119.64 (13)

$120.19(13)$

119.55 (13)

120.2

120.2

$121.10(13)$

119.5

119.5

$106.72(12)$

106.39 (13)

$105.47(12)$

112.97 (13)

$112.21(12)$

112.54 (12)

$179.55(12)$

-113.91 (16)

$69.52(18)$

69.90 (17)

$-106.67(15)$

$49.0(2)$

-130.33 (14)

$179.51(12)$

13.0 (2)

-166.89 (13)

1.4 (2)

$-178.54(13)$

-0.5 (2)

$-0.5(2)$

$-178.86(13)$

0.7 (2)

$179.06(13)$

$0.2(2)$

$-1.2(2)$

$178.73(13)$

159.37 (13) 


$\begin{array}{llll}\mathrm{C} 7-\mathrm{C} 8-\mathrm{C} 8 \mathrm{~A}-\mathrm{N} 1 & -177.60(14) & \mathrm{C} 423-\mathrm{C} 424-\mathrm{C} 427-\mathrm{F} 472 & -22.27(19) \\ \mathrm{C} 7-\mathrm{C} 8-\mathrm{C} 8 \mathrm{~A}-\mathrm{C} 4 \mathrm{~A} & 2.1(2) & \mathrm{C} 425-\mathrm{C} 424-\mathrm{C} 427-\mathrm{F} 473 & 38.67(19) \\ \mathrm{C} 5-\mathrm{C} 4 \mathrm{~A}-\mathrm{C} 8 \mathrm{~A}-\mathrm{N} 1 & 177.86(13) & \mathrm{C} 423-\mathrm{C} 424-\mathrm{C} 427-\mathrm{F} 473 & -142.98(13) \\ \mathrm{C} 4-\mathrm{C} 4 \mathrm{~A}-\mathrm{C} 8 \mathrm{~A}-\mathrm{N} 1 & -0.8(2) & \mathrm{C} 425-\mathrm{C} 424-\mathrm{C} 427-\mathrm{F} 471 & -80.11(17) \\ \mathrm{C} 5-\mathrm{C} 4 \mathrm{~A}-\mathrm{C} 8 \mathrm{~A}-\mathrm{C} 8 & -1.8(2) & \mathrm{C} 423-\mathrm{C} 424-\mathrm{C} 427-\mathrm{F} 471 & 98.24(16)\end{array}$

Hydrogen-bond geometry $\left(\AA,{ }^{\circ}\right)$

\begin{tabular}{lllll}
\hline$D-\mathrm{H}^{\prime} \cdots A$ & $D-\mathrm{H}$ & $\mathrm{H} \cdots A$ & $D \cdots A$ & $D-\mathrm{H} \cdots A$ \\
\hline $\mathrm{C} 41-\mathrm{H} 41 \cdots \mathrm{O} 31^{\mathrm{i}}$ & 0.95 & 2.42 & $3.3290(17)$ & 161 \\
$\mathrm{C} 426-\mathrm{H} 426 \cdots C g 3^{\mathrm{ii}}$ & 0.95 & 3.00 & $3.7621(15)$ & 138 \\
\hline
\end{tabular}

Symmetry codes: (i) $x, y+1, z$; (ii) $-x+1, y-1 / 2,-z+1 / 2$.

Ethyl (E)-4-[2-(4-methoxyphenyl)ethenyl]-2-methylquinoline-3-carboxylate (IV)

\section{Crystal data}

$\mathrm{C}_{22} \mathrm{H}_{21} \mathrm{NO}_{3}$

$M_{r}=347.40$

Triclinic, $P \overline{1}$

$a=9.5301(8) \AA$

$b=10.3513(8) \AA$

$c=10.3621(8) \AA$

$\alpha=65.374(3)^{\circ}$

$\beta=86.583(3)^{\circ}$

$\gamma=76.376(3)^{\circ}$

$V=902.23(13) \AA^{3}$

Data collection

Bruker D8 Venture diffractometer

Radiation source: INCOATEC high brilliance microfocus sealed tube

Multilayer mirror monochromator

$\varphi$ and $\omega$ scans

Absorption correction: multi-scan (SADABS; Bruker, 2016)

$T_{\min }=0.954, T_{\max }=0.996$

Refinement

Refinement on $F^{2}$

Least-squares matrix: full

$R\left[F^{2}>2 \sigma\left(F^{2}\right)\right]=0.052$

$w R\left(F^{2}\right)=0.128$

$S=1.07$

4158 reflections

238 parameters

0 restraints
$Z=2$

$F(000)=368$

$D_{\mathrm{x}}=1.279 \mathrm{Mg} \mathrm{m}^{-3}$

Mo $K \alpha$ radiation, $\lambda=0.71073 \AA$

Cell parameters from 4158 reflections

$\theta=2.2-27.5^{\circ}$

$\mu=0.09 \mathrm{~mm}^{-1}$

$T=100 \mathrm{~K}$

Needle, yellow

$0.30 \times 0.12 \times 0.05 \mathrm{~mm}$

44489 measured reflections

4158 independent reflections

3440 reflections with $I>2 \sigma(I)$

$R_{\text {int }}=0.053$

$\theta_{\text {max }}=27.5^{\circ}, \theta_{\min }=2.2^{\circ}$

$h=-12 \rightarrow 12$

$k=-13 \rightarrow 13$

$l=-13 \rightarrow 13$

Primary atom site location: difference Fourier map

Hydrogen site location: inferred from neighbouring sites

$\mathrm{H}$-atom parameters constrained

$w=1 /\left[\sigma^{2}\left(F_{\mathrm{o}}{ }^{2}\right)+(0.0422 P)^{2}+0.7299 P\right]$

where $P=\left(F_{\mathrm{o}}^{2}+2 F_{\mathrm{c}}^{2}\right) / 3$

$(\Delta / \sigma)_{\max }<0.001$

$\Delta \rho_{\max }=0.36 \mathrm{e} \AA^{-3}$

$\Delta \rho_{\min }=-0.21$ e $\AA^{-3}$ 


\section{Special details}

Geometry. All esds (except the esd in the dihedral angle between two 1.s. planes) are estimated using the full covariance matrix. The cell esds are taken into account individually in the estimation of esds in distances, angles and torsion angles; correlations between esds in cell parameters are only used when they are defined by crystal symmetry. An approximate (isotropic) treatment of cell esds is used for estimating esds involving l.s. planes.

Fractional atomic coordinates and isotropic or equivalent isotropic displacement parameters $\left(\AA^{2}\right)$

\begin{tabular}{|c|c|c|c|c|}
\hline & $x$ & $y$ & $z$ & $U_{\text {iso }} * / U_{\text {eq }}$ \\
\hline N1 & $0.81102(16)$ & $0.42663(16)$ & $0.95018(15)$ & $0.0239(3)$ \\
\hline $\mathrm{C} 2$ & $0.81545(18)$ & $0.31117(19)$ & $0.92506(17)$ & $0.0228(3)$ \\
\hline $\mathrm{C} 3$ & $0.70247(18)$ & $0.30251(18)$ & $0.84572(17)$ & $0.0206(3)$ \\
\hline $\mathrm{C} 4$ & $0.58290(17)$ & $0.41582(18)$ & $0.79378(16)$ & $0.0199(3)$ \\
\hline $\mathrm{C} 4 \mathrm{~A}$ & $0.57445(18)$ & $0.53964(18)$ & $0.82549(16)$ & $0.0204(3)$ \\
\hline $\mathrm{C} 5$ & $0.45312(19)$ & $0.65895(18)$ & $0.78548(17)$ & $0.0242(4)$ \\
\hline H5 & 0.3727 & 0.6588 & 0.7356 & $0.029 *$ \\
\hline C6 & $0.4503(2)$ & 0.77485 (19) & $0.81796(19)$ & $0.0291(4)$ \\
\hline H6 & 0.3683 & 0.8548 & 0.7901 & $0.035^{*}$ \\
\hline $\mathrm{C} 7$ & $0.5683(2)$ & $0.7762(2)$ & $0.89237(18)$ & $0.0294(4)$ \\
\hline H7 & 0.5659 & 0.8576 & 0.9136 & $0.035^{*}$ \\
\hline $\mathrm{C} 8$ & $0.6858(2)$ & $0.6619(2)$ & $0.93411(18)$ & $0.0272(4)$ \\
\hline $\mathrm{H} 8$ & 0.7647 & 0.6639 & 0.9846 & $0.033 *$ \\
\hline $\mathrm{C} 8 \mathrm{~A}$ & $0.69172(18)$ & $0.53980(19)$ & $0.90306(16)$ & $0.0221(3)$ \\
\hline $\mathrm{C} 21$ & $0.94750(19)$ & $0.1883(2)$ & $0.9796(2)$ & $0.0312(4)$ \\
\hline $\mathrm{H} 21 \mathrm{~A}$ & 0.9206 & 0.1020 & 1.0531 & $0.047 *$ \\
\hline $\mathrm{H} 21 \mathrm{~B}$ & 1.0182 & 0.2176 & 1.0202 & $0.047 *$ \\
\hline $\mathrm{H} 21 \mathrm{C}$ & 0.9899 & 0.1649 & 0.9012 & $0.047 *$ \\
\hline C31 & $0.72030(17)$ & $0.16500(19)$ & $0.82311(18)$ & $0.0221(3)$ \\
\hline $\mathrm{O} 31$ & $0.70662(14)$ & $0.05033(13)$ & $0.91571(13)$ & $0.0296(3)$ \\
\hline $\mathrm{O} 32$ & $0.75597(14)$ & $0.18596(14)$ & $0.69062(13)$ & $0.0286(3)$ \\
\hline C32 & $0.7718(2)$ & $0.0610(2)$ & $0.6539(2)$ & $0.0325(4)$ \\
\hline $\mathrm{H} 32 \mathrm{~A}$ & 0.8004 & -0.0312 & 0.7402 & $0.039 *$ \\
\hline H32B & 0.8486 & 0.0633 & 0.5848 & $0.039 *$ \\
\hline C33 & $0.6316(2)$ & $0.0661(2)$ & $0.59060(19)$ & $0.0317(4)$ \\
\hline $\mathrm{H} 33 \mathrm{~A}$ & 0.6002 & 0.1602 & 0.5089 & $0.048 *$ \\
\hline H33B & 0.5579 & 0.0548 & 0.6621 & $0.048^{*}$ \\
\hline $\mathrm{H} 33 \mathrm{C}$ & 0.6453 & -0.0132 & 0.5595 & $0.048^{*}$ \\
\hline $\mathrm{C} 41$ & $0.46640(17)$ & $0.41644(18)$ & $0.70556(17)$ & $0.0204(3)$ \\
\hline $\mathrm{H} 41$ & 0.4353 & 0.5012 & 0.6202 & $0.024 *$ \\
\hline $\mathrm{C} 42$ & $0.40176(17)$ & $0.30632(18)$ & $0.73726(17)$ & $0.0203(3)$ \\
\hline $\mathrm{H} 42$ & 0.4315 & 0.2241 & 0.8249 & $0.024 *$ \\
\hline $\mathrm{C} 421$ & $0.28969(17)$ & $0.29870(18)$ & $0.65120(16)$ & $0.0193(3)$ \\
\hline $\mathrm{C} 422$ & $0.23677(18)$ & $0.40931(19)$ & $0.51900(17)$ & $0.0230(3)$ \\
\hline $\mathrm{H} 422$ & 0.2688 & 0.4976 & 0.4846 & $0.028 *$ \\
\hline $\mathrm{C} 423$ & $0.13813(18)$ & $0.3931(2)$ & $0.43659(18)$ & $0.0251(4)$ \\
\hline $\mathrm{H} 423$ & 0.1030 & 0.4698 & 0.3469 & $0.030 *$ \\
\hline C424 & $0.09112(18)$ & $0.2639(2)$ & $0.48622(18)$ & $0.0242(4)$ \\
\hline $\mathrm{C} 425$ & 0.13894 (19) & $0.1541(2)$ & 0.61989 (19) & 0.0269 (4) \\
\hline
\end{tabular}


supporting information

\begin{tabular}{lllll}
$\mathrm{H} 425$ & 0.1046 & 0.0671 & 0.6552 & $0.032^{*}$ \\
$\mathrm{C} 426$ & $0.23657(18)$ & $0.17209(18)$ & $0.70116(18)$ & $0.0232(3)$ \\
$\mathrm{H} 426$ & 0.2681 & 0.0970 & 0.7926 & $0.028^{*}$ \\
$\mathrm{O} 424$ & $-0.00165(14)$ & $0.23441(16)$ & $0.41192(13)$ & $0.0334(3)$ \\
$\mathrm{C} 427$ & $-0.0515(2)$ & $0.3436(2)$ & $0.2731(2)$ & $0.0355(5)$ \\
$\mathrm{H} 47 \mathrm{~A}$ & 0.0316 & 0.3680 & 0.2155 & $0.053^{*}$ \\
$\mathrm{H} 47 \mathrm{~B}$ & -0.1105 & 0.4313 & 0.2807 & $0.053^{*}$ \\
$\mathrm{H} 47 \mathrm{C}$ & -0.1098 & 0.3061 & 0.2280 & $0.053^{*}$ \\
\hline
\end{tabular}

Atomic displacement parameters $\left(\AA^{2}\right)$

\begin{tabular}{lllllll}
\hline & $U^{11}$ & $U^{22}$ & $U^{33}$ & $U^{12}$ & $U^{13}$ & $U^{23}$ \\
\hline $\mathrm{N} 1$ & $0.0264(7)$ & $0.0304(8)$ & $0.0188(7)$ & $-0.0121(6)$ & $-0.0007(5)$ & $-0.0107(6)$ \\
$\mathrm{C} 2$ & $0.0238(8)$ & $0.0285(9)$ & $0.0175(8)$ & $-0.0093(7)$ & $-0.0003(6)$ & $-0.0090(7)$ \\
$\mathrm{C} 3$ & $0.0237(8)$ & $0.0236(8)$ & $0.0176(7)$ & $-0.0087(6)$ & $0.0008(6)$ & $-0.0097(6)$ \\
$\mathrm{C} 4$ & $0.0241(8)$ & $0.0232(8)$ & $0.0143(7)$ & $-0.0107(6)$ & $0.0011(6)$ & $-0.0070(6)$ \\
$\mathrm{C} 4 \mathrm{~A}$ & $0.0260(8)$ & $0.0222(8)$ & $0.0147(7)$ & $-0.0110(6)$ & $0.0035(6)$ & $-0.0068(6)$ \\
$\mathrm{C} 5$ & $0.0306(9)$ & $0.0222(8)$ & $0.0178(8)$ & $-0.0088(7)$ & $0.0010(6)$ & $-0.0050(6)$ \\
C6 & $0.0412(10)$ & $0.0209(9)$ & $0.0224(8)$ & $-0.0072(7)$ & $0.0024(7)$ & $-0.0064(7)$ \\
C7 & $0.0494(11)$ & $0.0226(9)$ & $0.0206(8)$ & $-0.0154(8)$ & $0.0057(7)$ & $-0.0100(7)$ \\
C8 & $0.0395(10)$ & $0.0307(9)$ & $0.0177(8)$ & $-0.0193(8)$ & $0.0031(7)$ & $-0.0106(7)$ \\
C8A & $0.0289(9)$ & $0.0263(8)$ & $0.0142(7)$ & $-0.0132(7)$ & $0.0036(6)$ & $-0.0082(6)$ \\
C21 & $0.0248(9)$ & $0.0369(10)$ & $0.0346(10)$ & $-0.0043(8)$ & $-0.0078(7)$ & $-0.0179(8)$ \\
C31 & $0.0176(7)$ & $0.0263(9)$ & $0.0246(8)$ & $-0.0047(6)$ & $-0.0033(6)$ & $-0.0125(7)$ \\
O31 & $0.0380(7)$ & $0.0229(6)$ & $0.0270(7)$ & $-0.0067(5)$ & $-0.0055(5)$ & $-0.0087(5)$ \\
O32 & $0.0323(7)$ & $0.0346(7)$ & $0.0294(7)$ & $-0.0138(6)$ & $0.0065(5)$ & $-0.0208(6)$ \\
C32 & $0.0321(10)$ & $0.0394(11)$ & $0.0409(11)$ & $-0.0128(8)$ & $0.0086(8)$ & $-0.0297(9)$ \\
C33 & $0.0440(11)$ & $0.0314(10)$ & $0.0246(9)$ & $-0.0154(8)$ & $-0.0012(8)$ & $-0.0126(8)$ \\
C41 & $0.0221(8)$ & $0.0227(8)$ & $0.0161(7)$ & $-0.0040(6)$ & $-0.0029(6)$ & $-0.0079(6)$ \\
C42 & $0.0205(8)$ & $0.0231(8)$ & $0.0168(7)$ & $-0.0031(6)$ & $-0.0021(6)$ & $-0.0086(6)$ \\
C421 & $0.0187(7)$ & $0.0231(8)$ & $0.0180(7)$ & $-0.0043(6)$ & $-0.0005(6)$ & $-0.0104(6)$ \\
C422 & $0.0250(8)$ & $0.0256(9)$ & $0.0188(8)$ & $-0.0090(7)$ & $-0.0002(6)$ & $-0.0078(7)$ \\
C423 & $0.0235(8)$ & $0.0317(9)$ & $0.0180(8)$ & $-0.0059(7)$ & $-0.0025(6)$ & $-0.0082(7)$ \\
C424 & $0.0186(8)$ & $0.0366(10)$ & $0.0221(8)$ & $-0.0085(7)$ & $-0.0007(6)$ & $-0.0155(7)$ \\
C425 & $0.0264(9)$ & $0.0286(9)$ & $0.0276(9)$ & $-0.0117(7)$ & $-0.0020(7)$ & $-0.0103(7)$ \\
C426 & $0.0235(8)$ & $0.0234(8)$ & $0.0204(8)$ & $-0.0055(7)$ & $-0.0033(6)$ & $-0.0064(7)$ \\
O424 & $0.0319(7)$ & $0.0486(8)$ & $0.0252(7)$ & $-0.0185(6)$ & $-0.0057(5)$ & $-0.0150(6)$ \\
C427 & $0.0259(9)$ & $0.0599(13)$ & $0.0257(9)$ & $-0.0122(9)$ & $-0.0055(7)$ & $-0.0205(9)$ \\
& & & & & & \\
& & & & & &
\end{tabular}

Geometric parameters $\left(\AA,{ }^{o}\right)$

\begin{tabular}{llll}
\hline $\mathrm{N} 1-\mathrm{C} 2$ & $1.316(2)$ & $\mathrm{C} 32-\mathrm{H} 32 \mathrm{~A}$ & 0.9900 \\
$\mathrm{~N} 1-\mathrm{C} 8 \mathrm{~A}$ & $1.366(2)$ & $\mathrm{C} 32-\mathrm{H} 32 \mathrm{~B}$ & 0.9900 \\
$\mathrm{C} 2-\mathrm{C} 3$ & $1.434(2)$ & $\mathrm{C} 33-\mathrm{H} 33 \mathrm{~A}$ & 0.9800 \\
$\mathrm{C} 2-\mathrm{C} 21$ & $1.501(2)$ & $\mathrm{C} 33-\mathrm{H} 33 \mathrm{~B}$ & 0.9800 \\
$\mathrm{C} 3-\mathrm{C} 4$ & $1.372(2)$ & $\mathrm{C} 33-\mathrm{H} 33 \mathrm{C}$ & 0.9800 \\
$\mathrm{C} 3-\mathrm{C} 31$ & $1.506(2)$ & $\mathrm{C} 41-\mathrm{C} 42$ & $1.335(2)$ \\
$\mathrm{C} 4-\mathrm{C} 4 \mathrm{~A}$ & $1.434(2)$ & $\mathrm{C} 41-\mathrm{H} 41$ & 0.9500
\end{tabular}




\begin{tabular}{|c|c|c|c|}
\hline $\mathrm{C} 4-\mathrm{C} 41$ & $1.477(2)$ & $\mathrm{C} 42-\mathrm{C} 421$ & $1.468(2)$ \\
\hline $\mathrm{C} 4 \mathrm{~A}-\mathrm{C} 5$ & $1.411(2)$ & $\mathrm{C} 42-\mathrm{H} 42$ & 0.9500 \\
\hline $\mathrm{C} 4 \mathrm{~A}-\mathrm{C} 8 \mathrm{~A}$ & $1.416(2)$ & $\mathrm{C} 421-\mathrm{C} 422$ & $1.395(2)$ \\
\hline $\mathrm{C} 5-\mathrm{C} 6$ & $1.369(2)$ & $\mathrm{C} 421-\mathrm{C} 426$ & $1.399(2)$ \\
\hline $\mathrm{C} 5-\mathrm{H} 5$ & 0.9500 & $\mathrm{C} 422-\mathrm{C} 423$ & $1.389(2)$ \\
\hline $\mathrm{C} 6-\mathrm{C} 7$ & $1.407(3)$ & $\mathrm{C} 422-\mathrm{H} 422$ & 0.9500 \\
\hline $\mathrm{C} 6-\mathrm{H} 6$ & 0.9500 & C423-C424 & $1.391(2)$ \\
\hline $\mathrm{C} 7-\mathrm{C} 8$ & $1.359(3)$ & $\mathrm{C} 423-\mathrm{H} 423$ & 0.9500 \\
\hline $\mathrm{C} 7-\mathrm{H} 7$ & 0.9500 & $\mathrm{C} 424-\mathrm{O} 424$ & $1.3682(19)$ \\
\hline $\mathrm{C} 8-\mathrm{C} 8 \mathrm{~A}$ & $1.417(2)$ & $\mathrm{C} 424-\mathrm{C} 425$ & $1.392(2)$ \\
\hline $\mathrm{C} 8-\mathrm{H} 8$ & 0.9500 & $\mathrm{C} 425-\mathrm{C} 426$ & $1.384(2)$ \\
\hline $\mathrm{C} 21-\mathrm{H} 21 \mathrm{~A}$ & 0.9800 & $\mathrm{C} 425-\mathrm{H} 425$ & 0.9500 \\
\hline $\mathrm{C} 21-\mathrm{H} 21 \mathrm{~B}$ & 0.9800 & $\mathrm{C} 426-\mathrm{H} 426$ & 0.9500 \\
\hline $\mathrm{C} 21-\mathrm{H} 21 \mathrm{C}$ & 0.9800 & $\mathrm{O} 424-\mathrm{C} 427$ & $1.430(2)$ \\
\hline $\mathrm{C} 31-\mathrm{O} 31$ & $1.206(2)$ & $\mathrm{C} 427-\mathrm{H} 47 \mathrm{~A}$ & 0.9800 \\
\hline $\mathrm{C} 31-\mathrm{O} 32$ & $1.334(2)$ & С $427-\mathrm{H} 47 \mathrm{~B}$ & 0.9800 \\
\hline $\mathrm{O} 32-\mathrm{C} 32$ & $1.467(2)$ & $\mathrm{C} 427-\mathrm{H} 47 \mathrm{C}$ & 0.9800 \\
\hline $\mathrm{C} 32-\mathrm{C} 33$ & $1.503(3)$ & & \\
\hline $\mathrm{C} 2-\mathrm{N} 1-\mathrm{C} 8 \mathrm{~A}$ & $118.47(14)$ & $\mathrm{O} 32-\mathrm{C} 32-\mathrm{H} 32 \mathrm{~B}$ & 109.6 \\
\hline $\mathrm{N} 1-\mathrm{C} 2-\mathrm{C} 3$ & $122.42(16)$ & $\mathrm{C} 33-\mathrm{C} 32-\mathrm{H} 32 \mathrm{~B}$ & 109.6 \\
\hline $\mathrm{N} 1-\mathrm{C} 2-\mathrm{C} 21$ & $117.03(15)$ & $\mathrm{H} 32 \mathrm{~A}-\mathrm{C} 32-\mathrm{H} 32 \mathrm{~B}$ & 108.2 \\
\hline $\mathrm{C} 3-\mathrm{C} 2-\mathrm{C} 21$ & $120.52(15)$ & $\mathrm{C} 32-\mathrm{C} 33-\mathrm{H} 33 \mathrm{~A}$ & 109.5 \\
\hline $\mathrm{C} 4-\mathrm{C} 3-\mathrm{C} 2$ & $120.55(15)$ & $\mathrm{C} 32-\mathrm{C} 33-\mathrm{H} 33 \mathrm{~B}$ & 109.5 \\
\hline $\mathrm{C} 4-\mathrm{C} 3-\mathrm{C} 31$ & $121.94(14)$ & $\mathrm{H} 33 \mathrm{~A}-\mathrm{C} 33-\mathrm{H} 33 \mathrm{~B}$ & 109.5 \\
\hline $\mathrm{C} 2-\mathrm{C} 3-\mathrm{C} 31$ & $117.51(15)$ & $\mathrm{C} 32-\mathrm{C} 33-\mathrm{H} 33 \mathrm{C}$ & 109.5 \\
\hline $\mathrm{C} 3-\mathrm{C} 4-\mathrm{C} 4 \mathrm{~A}$ & $117.41(14)$ & $\mathrm{H} 33 \mathrm{~A}-\mathrm{C} 33-\mathrm{H} 33 \mathrm{C}$ & 109.5 \\
\hline $\mathrm{C} 3-\mathrm{C} 4-\mathrm{C} 41$ & $123.09(14)$ & $\mathrm{H} 33 \mathrm{~B}-\mathrm{C} 33-\mathrm{H} 33 \mathrm{C}$ & 109.5 \\
\hline $\mathrm{C} 4 \mathrm{~A}-\mathrm{C} 4-\mathrm{C} 41$ & $119.49(15)$ & $\mathrm{C} 42-\mathrm{C} 41-\mathrm{C} 4$ & $124.64(15)$ \\
\hline $\mathrm{C} 5-\mathrm{C} 4 \mathrm{~A}-\mathrm{C} 8 \mathrm{~A}$ & $118.99(15)$ & $\mathrm{C} 42-\mathrm{C} 41-\mathrm{H} 41$ & 117.7 \\
\hline $\mathrm{C} 5-\mathrm{C} 4 \mathrm{~A}-\mathrm{C} 4$ & $122.73(15)$ & $\mathrm{C} 4-\mathrm{C} 41-\mathrm{H} 41$ & 117.7 \\
\hline $\mathrm{C} 8 \mathrm{~A}-\mathrm{C} 4 \mathrm{~A}-\mathrm{C} 4$ & $118.26(15)$ & $\mathrm{C} 41-\mathrm{C} 42-\mathrm{C} 421$ & $126.96(15)$ \\
\hline $\mathrm{C} 6-\mathrm{C} 5-\mathrm{C} 4 \mathrm{~A}$ & $120.54(16)$ & $\mathrm{C} 41-\mathrm{C} 42-\mathrm{H} 42$ & 116.5 \\
\hline $\mathrm{C} 6-\mathrm{C} 5-\mathrm{H} 5$ & 119.7 & $\mathrm{C} 421-\mathrm{C} 42-\mathrm{H} 42$ & 116.5 \\
\hline $\mathrm{C} 4 \mathrm{~A}-\mathrm{C} 5-\mathrm{H} 5$ & 119.7 & $\mathrm{C} 422-\mathrm{C} 421-\mathrm{C} 426$ & $117.94(14)$ \\
\hline $\mathrm{C} 5-\mathrm{C} 6-\mathrm{C} 7$ & $120.35(17)$ & $\mathrm{C} 422-\mathrm{C} 421-\mathrm{C} 42$ & $123.34(15)$ \\
\hline $\mathrm{C} 5-\mathrm{C} 6-\mathrm{H} 6$ & 119.8 & $\mathrm{C} 426-\mathrm{C} 421-\mathrm{C} 42$ & $118.68(14)$ \\
\hline $\mathrm{C} 7-\mathrm{C} 6-\mathrm{H} 6$ & 119.8 & $\mathrm{C} 423-\mathrm{C} 422-\mathrm{C} 421$ & $121.39(16)$ \\
\hline $\mathrm{C} 8-\mathrm{C} 7-\mathrm{C} 6$ & $120.45(16)$ & $\mathrm{C} 423-\mathrm{C} 422-\mathrm{H} 422$ & 119.3 \\
\hline $\mathrm{C} 8-\mathrm{C} 7-\mathrm{H} 7$ & 119.8 & $\mathrm{C} 421-\mathrm{C} 422-\mathrm{H} 422$ & 119.3 \\
\hline $\mathrm{C} 6-\mathrm{C} 7-\mathrm{H} 7$ & 119.8 & $\mathrm{C} 422-\mathrm{C} 423-\mathrm{C} 424$ & $119.57(16)$ \\
\hline $\mathrm{C} 7-\mathrm{C} 8-\mathrm{C} 8 \mathrm{~A}$ & $120.61(16)$ & $\mathrm{C} 422-\mathrm{C} 423-\mathrm{H} 423$ & 120.2 \\
\hline $\mathrm{C} 7-\mathrm{C} 8-\mathrm{H} 8$ & 119.7 & $\mathrm{C} 424-\mathrm{C} 423-\mathrm{H} 423$ & 120.2 \\
\hline $\mathrm{C} 8 \mathrm{~A}-\mathrm{C} 8-\mathrm{H} 8$ & 119.7 & $\mathrm{O} 424-\mathrm{C} 424-\mathrm{C} 423$ & $124.44(16)$ \\
\hline $\mathrm{N} 1-\mathrm{C} 8 \mathrm{~A}-\mathrm{C} 4 \mathrm{~A}$ & $122.83(15)$ & $\mathrm{O} 424-\mathrm{C} 424-\mathrm{C} 425$ & $115.64(16)$ \\
\hline $\mathrm{N} 1-\mathrm{C} 8 \mathrm{~A}-\mathrm{C} 8$ & $118.15(15)$ & $\mathrm{C} 423-\mathrm{C} 424-\mathrm{C} 425$ & $119.92(15)$ \\
\hline $\mathrm{C} 4 \mathrm{~A}-\mathrm{C} 8 \mathrm{~A}-\mathrm{C} 8$ & $119.02(16)$ & $\mathrm{C} 426-\mathrm{C} 425-\mathrm{C} 424$ & $119.84(16)$ \\
\hline $\mathrm{C} 2-\mathrm{C} 21-\mathrm{H} 21 \mathrm{~A}$ & 109.5 & $\mathrm{C} 426-\mathrm{C} 425-\mathrm{H} 425$ & 120.1 \\
\hline
\end{tabular}




\begin{tabular}{|c|c|c|c|}
\hline $\mathrm{C} 2-\mathrm{C} 21-\mathrm{H} 21 \mathrm{~B}$ & 109.5 & $\mathrm{C} 424-\mathrm{C} 425-\mathrm{H} 425$ & 120.1 \\
\hline $\mathrm{H} 21 \mathrm{~A}-\mathrm{C} 21-\mathrm{H} 21 \mathrm{~B}$ & 109.5 & $\mathrm{C} 425-\mathrm{C} 426-\mathrm{C} 421$ & $121.25(16)$ \\
\hline $\mathrm{C} 2-\mathrm{C} 21-\mathrm{H} 21 \mathrm{C}$ & 109.5 & $\mathrm{C} 425-\mathrm{C} 426-\mathrm{H} 426$ & 119.4 \\
\hline $\mathrm{H} 21 \mathrm{~A}-\mathrm{C} 21-\mathrm{H} 21 \mathrm{C}$ & 109.5 & $\mathrm{C} 421-\mathrm{C} 426-\mathrm{H} 426$ & 119.4 \\
\hline $\mathrm{H} 21 \mathrm{~B}-\mathrm{C} 21-\mathrm{H} 21 \mathrm{C}$ & 109.5 & $\mathrm{C} 424-\mathrm{O} 424-\mathrm{C} 427$ & $117.37(15)$ \\
\hline $\mathrm{O} 31-\mathrm{C} 31-\mathrm{O} 32$ & $125.09(15)$ & $\mathrm{O} 424-\mathrm{C} 427-\mathrm{H} 47 \mathrm{~A}$ & 109.5 \\
\hline $\mathrm{O} 31-\mathrm{C} 31-\mathrm{C} 3$ & $123.28(15)$ & $\mathrm{O} 424-\mathrm{C} 427-\mathrm{H} 47 \mathrm{~B}$ & 109.5 \\
\hline $\mathrm{O} 32-\mathrm{C} 31-\mathrm{C} 3$ & $111.61(14)$ & H47A-C427-H47B & 109.5 \\
\hline $\mathrm{C} 31-\mathrm{O} 32-\mathrm{C} 32$ & $116.83(14)$ & $\mathrm{O} 424-\mathrm{C} 427-\mathrm{H} 47 \mathrm{C}$ & 109.5 \\
\hline $\mathrm{O} 32-\mathrm{C} 32-\mathrm{C} 33$ & $110.10(15)$ & $\mathrm{H} 47 \mathrm{~A}-\mathrm{C} 427-\mathrm{H} 47 \mathrm{C}$ & 109.5 \\
\hline $\mathrm{O} 32-\mathrm{C} 32-\mathrm{H} 32 \mathrm{~A}$ & 109.6 & $\mathrm{H} 47 \mathrm{~B}-\mathrm{C} 427-\mathrm{H} 47 \mathrm{C}$ & 109.5 \\
\hline $\mathrm{C} 33-\mathrm{C} 32-\mathrm{H} 32 \mathrm{~A}$ & 109.6 & & \\
\hline $\mathrm{C} 8 \mathrm{~A}-\mathrm{N} 1-\mathrm{C} 2-\mathrm{C} 3$ & $2.4(2)$ & $\mathrm{C} 7-\mathrm{C} 8-\mathrm{C} 8 \mathrm{~A}-\mathrm{C} 4 \mathrm{~A}$ & $1.2(2)$ \\
\hline $\mathrm{C} 8 \mathrm{~A}-\mathrm{N} 1-\mathrm{C} 2-\mathrm{C} 21$ & $-179.40(15)$ & $\mathrm{C} 4-\mathrm{C} 3-\mathrm{C} 31-\mathrm{O} 31$ & $-106.0(2)$ \\
\hline $\mathrm{N} 1-\mathrm{C} 2-\mathrm{C} 3-\mathrm{C} 4$ & $-0.8(3)$ & $\mathrm{C} 2-\mathrm{C} 3-\mathrm{C} 31-\mathrm{O} 31$ & $73.7(2)$ \\
\hline $\mathrm{C} 21-\mathrm{C} 2-\mathrm{C} 3-\mathrm{C} 4$ & $-178.89(16)$ & $\mathrm{C} 4-\mathrm{C} 3-\mathrm{C} 31-\mathrm{O} 32$ & $75.5(2)$ \\
\hline $\mathrm{N} 1-\mathrm{C} 2-\mathrm{C} 3-\mathrm{C} 31$ & $179.59(15)$ & $\mathrm{C} 2-\mathrm{C} 3-\mathrm{C} 31-\mathrm{O} 32$ & $-104.85(17)$ \\
\hline $\mathrm{C} 21-\mathrm{C} 2-\mathrm{C} 3-\mathrm{C} 31$ & $1.5(2)$ & $\mathrm{O} 31-\mathrm{C} 31-\mathrm{O} 32-\mathrm{C} 32$ & $3.5(2)$ \\
\hline $\mathrm{C} 2-\mathrm{C} 3-\mathrm{C} 4-\mathrm{C} 4 \mathrm{~A}$ & $-1.7(2)$ & $\mathrm{C} 3-\mathrm{C} 31-\mathrm{O} 32-\mathrm{C} 32$ & $-178.03(14)$ \\
\hline $\mathrm{C} 31-\mathrm{C} 3-\mathrm{C} 4-\mathrm{C} 4 \mathrm{~A}$ & $177.92(14)$ & $\mathrm{C} 31-\mathrm{O} 32-\mathrm{C} 32-\mathrm{C} 33$ & $93.04(19)$ \\
\hline $\mathrm{C} 2-\mathrm{C} 3-\mathrm{C} 4-\mathrm{C} 41$ & $176.96(15)$ & $\mathrm{C} 3-\mathrm{C} 4-\mathrm{C} 41-\mathrm{C} 42$ & $48.8(2)$ \\
\hline $\mathrm{C} 31-\mathrm{C} 3-\mathrm{C} 4-\mathrm{C} 41$ & $-3.4(2)$ & $\mathrm{C} 4 \mathrm{~A}-\mathrm{C} 4-\mathrm{C} 41-\mathrm{C} 42$ & $-132.53(17)$ \\
\hline $\mathrm{C} 3-\mathrm{C} 4-\mathrm{C} 4 \mathrm{~A}-\mathrm{C} 5$ & $-176.06(15)$ & $\mathrm{C} 4-\mathrm{C} 41-\mathrm{C} 42-\mathrm{C} 421$ & $-177.48(15)$ \\
\hline $\mathrm{C} 41-\mathrm{C} 4-\mathrm{C} 4 \mathrm{~A}-\mathrm{C} 5$ & $5.2(2)$ & $\mathrm{C} 41-\mathrm{C} 42-\mathrm{C} 421-\mathrm{C} 422$ & $1.2(3)$ \\
\hline $\mathrm{C} 3-\mathrm{C} 4-\mathrm{C} 4 \mathrm{~A}-\mathrm{C} 8 \mathrm{~A}$ & $2.4(2)$ & $\mathrm{C} 41-\mathrm{C} 42-\mathrm{C} 421-\mathrm{C} 426$ & $178.77(16)$ \\
\hline $\mathrm{C} 41-\mathrm{C} 4-\mathrm{C} 4 \mathrm{~A}-\mathrm{C} 8 \mathrm{~A}$ & $-176.28(14)$ & $\mathrm{C} 426-\mathrm{C} 421-\mathrm{C} 422-\mathrm{C} 423$ & $-2.2(2)$ \\
\hline $\mathrm{C} 8 \mathrm{~A}-\mathrm{C} 4 \mathrm{~A}-\mathrm{C} 5-\mathrm{C} 6$ & $1.7(2)$ & $\mathrm{C} 42-\mathrm{C} 421-\mathrm{C} 422-\mathrm{C} 423$ & $175.34(15)$ \\
\hline $\mathrm{C} 4-\mathrm{C} 4 \mathrm{~A}-\mathrm{C} 5-\mathrm{C} 6$ & $-179.86(16)$ & $\mathrm{C} 421-\mathrm{C} 422-\mathrm{C} 423-\mathrm{C} 424$ & $-0.2(3)$ \\
\hline $\mathrm{C} 4 \mathrm{~A}-\mathrm{C} 5-\mathrm{C} 6-\mathrm{C} 7$ & $-0.3(3)$ & $\mathrm{C} 422-\mathrm{C} 423-\mathrm{C} 424-\mathrm{O} 424$ & $-177.63(16)$ \\
\hline $\mathrm{C} 5-\mathrm{C} 6-\mathrm{C} 7-\mathrm{C} 8$ & $-0.7(3)$ & $\mathrm{C} 422-\mathrm{C} 423-\mathrm{C} 424-\mathrm{C} 425$ & $2.3(3)$ \\
\hline $\mathrm{C} 6-\mathrm{C} 7-\mathrm{C} 8-\mathrm{C} 8 \mathrm{~A}$ & $0.2(3)$ & $\mathrm{O} 424-\mathrm{C} 424-\mathrm{C} 425-\mathrm{C} 426$ & $177.99(16)$ \\
\hline $\mathrm{C} 2-\mathrm{N} 1-\mathrm{C} 8 \mathrm{~A}-\mathrm{C} 4 \mathrm{~A}$ & $-1.6(2)$ & $\mathrm{C} 423-\mathrm{C} 424-\mathrm{C} 425-\mathrm{C} 426$ & $-2.0(3)$ \\
\hline $\mathrm{C} 2-\mathrm{N} 1-\mathrm{C} 8 \mathrm{~A}-\mathrm{C} 8$ & $178.18(15)$ & $\mathrm{C} 424-\mathrm{C} 425-\mathrm{C} 426-\mathrm{C} 421$ & $-0.5(3)$ \\
\hline $\mathrm{C} 5-\mathrm{C} 4 \mathrm{~A}-\mathrm{C} 8 \mathrm{~A}-\mathrm{N} 1$ & $177.69(15)$ & $\mathrm{C} 422-\mathrm{C} 421-\mathrm{C} 426-\mathrm{C} 425$ & $2.6(2)$ \\
\hline $\mathrm{C} 4-\mathrm{C} 4 \mathrm{~A}-\mathrm{C} 8 \mathrm{~A}-\mathrm{N} 1$ & $-0.9(2)$ & $\mathrm{C} 42-\mathrm{C} 421-\mathrm{C} 426-\mathrm{C} 425$ & $-175.09(15)$ \\
\hline $\mathrm{C} 5-\mathrm{C} 4 \mathrm{~A}-\mathrm{C} 8 \mathrm{~A}-\mathrm{C} 8$ & $-2.1(2)$ & $\mathrm{C} 423-\mathrm{C} 424-\mathrm{O} 424-\mathrm{C} 427$ & $0.9(3)$ \\
\hline $\mathrm{C} 4-\mathrm{C} 4 \mathrm{~A}-\mathrm{C} 8 \mathrm{~A}-\mathrm{C} 8$ & $179.38(15)$ & $\mathrm{C} 425-\mathrm{C} 424-\mathrm{O} 424-\mathrm{C} 427$ & $-179.02(16)$ \\
\hline $\mathrm{C} 7-\mathrm{C} 8-\mathrm{C} 8 \mathrm{~A}-\mathrm{N} 1$ & $-178.61(16)$ & & \\
\hline
\end{tabular}

Hydrogen-bond geometry $\left(A,{ }^{\circ}\right)$

\begin{tabular}{lllll}
\hline$D-\mathrm{H} \cdots A$ & $D-\mathrm{H}$ & $\mathrm{H} \cdots A$ & $D \cdots A$ & $D-\mathrm{H} \cdots A$ \\
\hline $\mathrm{C} 32-\mathrm{H} 32 B \cdots \mathrm{O} 424^{\mathrm{i}}$ & 0.99 & 2.57 & $3.406(2)$ & 142 \\
$\mathrm{C} 423-\mathrm{H} 423 \cdots C g 1^{\mathrm{ii}}$ & 0.95 & 2.91 & $3.4894(19)$ & 120 \\
\hline
\end{tabular}

Symmetry codes: (i) $x+1, y, z$; (ii) $-x+1,-y+1,-z+1$. 
Ethyl (E)-4-[2-(4-bromophenyl)ethenyl]-2-methylquinoline-3-carboxylate (V)

Crystal data

$\mathrm{C}_{21} \mathrm{H}_{18} \mathrm{BrNO}_{2}$

$M_{r}=396.26$

Monoclinic, $P 2{ }_{1} / n$

$a=9.5709(6) \AA$

$b=10.6119(7) \AA$

$c=18.2074(10) \AA$

$\beta=90.939(2)^{\circ}$

$V=1849.0(2) \AA^{3}$

$Z=4$

\section{Data collection}

Bruker D8 Venture diffractometer

Radiation source: INCOATEC high brilliance microfocus sealed tube

Multilayer mirror monochromator

$\varphi$ and $\omega$ scans

Absorption correction: multi-scan (SADABS; Bruker, 2016)

$T_{\min }=0.595, T_{\max }=0.715$

\section{Refinement}

Refinement on $F^{2}$

Least-squares matrix: full

$R\left[F^{2}>2 \sigma\left(F^{2}\right)\right]=0.022$

$w R\left(F^{2}\right)=0.056$

$S=1.02$

4588 reflections

228 parameters

0 restraints
$F(000)=808$

$D_{\mathrm{x}}=1.424 \mathrm{Mg} \mathrm{m}^{-3}$

Mo $K \alpha$ radiation, $\lambda=0.71073 \AA$

Cell parameters from 4558 reflections

$\theta=2.2-28.3^{\circ}$

$\mu=2.24 \mathrm{~mm}^{-1}$

$T=100 \mathrm{~K}$

Block, yellow

$0.25 \times 0.18 \times 0.15 \mathrm{~mm}$

54534 measured reflections

4588 independent reflections

4098 reflections with $I>2 \sigma(I)$

$R_{\text {int }}=0.041$

$\theta_{\max }=28.3^{\circ}, \theta_{\min }=2.2^{\circ}$

$h=-12 \rightarrow 12$

$k=-14 \rightarrow 14$

$l=-24 \rightarrow 24$
Primary atom site location: difference Fourier map

Hydrogen site location: inferred from neighbouring sites

$\mathrm{H}$-atom parameters constrained

$w=1 /\left[\sigma^{2}\left(F_{\mathrm{o}}{ }^{2}\right)+(0.0237 P)^{2}+1.0895 P\right]$

where $P=\left(F_{\mathrm{o}}^{2}+2 F_{\mathrm{c}}^{2}\right) / 3$

$(\Delta / \sigma)_{\max }=0.003$

$\Delta \rho_{\max }=0.35$ e $\AA^{-3}$

$\Delta \rho_{\min }=-0.42$ e $\AA^{-3}$

Special details

Geometry. All esds (except the esd in the dihedral angle between two 1.s. planes) are estimated using the full covariance matrix. The cell esds are taken into account individually in the estimation of esds in distances, angles and torsion angles; correlations between esds in cell parameters are only used when they are defined by crystal symmetry. An approximate (isotropic) treatment of cell esds is used for estimating esds involving 1.s. planes.

Fractional atomic coordinates and isotropic or equivalent isotropic displacement parameters $\left(\AA^{2}\right)$

\begin{tabular}{lllll}
\hline & $x$ & $y$ & $z$ & $U_{\text {iso }} * / U_{\text {eq }}$ \\
\hline N1 & $0.22794(12)$ & $0.20653(11)$ & $0.63602(6)$ & $0.0186(2)$ \\
C2 & $0.36010(14)$ & $0.19499(13)$ & $0.61835(7)$ & $0.0179(3)$ \\
C3 & $0.45699(14)$ & $0.29687(13)$ & $0.62494(7)$ & $0.0161(2)$ \\
C4 & $0.41542(14)$ & $0.41110(12)$ & $0.65325(7)$ & $0.0156(2)$ \\
C4A & $0.27233(14)$ & $0.42464(13)$ & $0.67343(7)$ & $0.0165(3)$ \\
C5 & $0.21593(15)$ & $0.53906(14)$ & $0.70002(8)$ & $0.0211(3)$ \\
H5 & 0.2756 & 0.6092 & 0.7086 & $0.025^{*}$ \\
C6 & $0.07589(16)$ & $0.54916(15)$ & $0.71338(8)$ & $0.0249(3)$
\end{tabular}




$\begin{array}{lllll}\text { H6 } & 0.0390 & 0.6264 & 0.7309 & 0.030^{*} \\ \text { C7 } & -0.01353(16) & 0.44582(15) & 0.70130(9) & 0.0261(3) \\ \text { H7 } & -0.1106 & 0.4540 & 0.7104 & 0.031^{*} \\ \text { C8 } & 0.03799(15) & 0.33360(14) & 0.67658(8) & 0.0225(3) \\ \text { H8 } & -0.0232 & 0.2641 & 0.6692 & 0.027^{*} \\ \text { C8A } & 0.18202(14) & 0.32044(13) & 0.66196(7) & 0.0172(3) \\ \text { C21 } & 0.40674(16) & 0.06875(14) & 0.59035(9) & 0.0269(3) \\ \text { H21A } & 0.3382 & 0.0044 & 0.6036 & 0.040^{*} \\ \text { H21B } & 0.4978 & 0.0475 & 0.6124 & 0.040^{*} \\ \text { H21C } & 0.4146 & 0.0721 & 0.5368 & 0.040^{*} \\ \text { C31 } & 0.60558(14) & 0.27447(13) & 0.60265(7) & 0.0181(3) \\ \text { O31 } & 0.70170(11) & 0.25715(11) & 0.64455(6) & 0.0248(2) \\ \text { O32 } & 0.61517(11) & 0.27338(11) & 0.52926(5) & 0.0241(2) \\ \text { C32 } & 0.75437(17) & 0.25167(17) & 0.49963(9) & 0.0306(3) \\ \text { H32A } & 0.7455 & 0.2142 & 0.4500 & 0.037^{*} \\ \text { H32B } & 0.8060 & 0.1914 & 0.5315 & 0.037^{*} \\ \text { C33 } & 0.83439(18) & 0.37338(18) & 0.49542(9) & 0.0339(4) \\ \text { H33A } & 0.7802 & 0.4348 & 0.4666 & 0.051^{*} \\ \text { H33B } & 0.9241 & 0.3584 & 0.4718 & 0.051^{*} \\ \text { H33C } & 0.8510 & 0.4062 & 0.5451 & 0.051^{*} \\ \text { C41 } & 0.51187(14) & 0.51853(12) & 0.66208(7) & 0.0168(3) \\ \text { H41 } & 0.5177 & 0.5591 & 0.7086 & 0.020^{*} \\ \text { C42 } & 0.59134(15) & 0.56169(13) & 0.60807(7) & 0.0185(3) \\ \text { H42 } & 0.5825 & 0.5209 & 0.5618 & 0.022^{*} \\ \text { C421 } & 0.69122(14) & 0.66634(13) & 0.61359(7) & 0.0174(3) \\ \text { C422 } & 0.73110(15) & 0.72094(13) & 0.68058(7) & 0.0189(3) \\ \text { H422 } & 0.6918 & 0.6908 & 0.7248 & 0.023^{*} \\ \text { C423 } & 0.82710(14) & 0.81836(13) & 0.68316(7) & 0.0188(3) \\ \text { H423 } & 0.8536 & 0.8552 & 0.7289 & 0.023^{*} \\ \text { C424 } & 0.88435(14) & 0.86183(12) & 0.61828(7) & 0.0176(3) \\ \text { Br42 } & 1.02007(2) & 0.99228(2) & 0.62329(2) & 0.02109(5) \\ \text { C425 } & 0.84705(16) & 0.81022(14) & 0.55109(8) & 0.0235(3) \\ \text { H425 } & 0.8861 & 0.8412 & 0.5070 & 0.028^{*} \\ \text { C426 } & 0.75115(16) & 0.71199(14) & 0.54958(8) & 0.0235(3) \\ \text { H426 } & 0.7257 & 0.6750 & 0.5038 & 0.028^{*} \\ & & & & \end{array}$

Atomic displacement parameters $\left(\AA^{2}\right)$

\begin{tabular}{lllllll}
\hline & $U^{11}$ & $U^{22}$ & $U^{33}$ & $U^{12}$ & $U^{13}$ & $U^{23}$ \\
\hline $\mathrm{N} 1$ & $0.0177(6)$ & $0.0175(5)$ & $0.0206(6)$ & $-0.0064(4)$ & $0.0008(4)$ & $0.0001(4)$ \\
$\mathrm{C} 2$ & $0.0191(7)$ & $0.0169(6)$ & $0.0178(6)$ & $-0.0046(5)$ & $0.0003(5)$ & $-0.0006(5)$ \\
$\mathrm{C} 3$ & $0.0147(6)$ & $0.0187(6)$ & $0.0149(6)$ & $-0.0045(5)$ & $0.0009(5)$ & $0.0007(5)$ \\
$\mathrm{C} 4$ & $0.0158(6)$ & $0.0170(6)$ & $0.0138(6)$ & $-0.0049(5)$ & $-0.0018(5)$ & $0.0018(5)$ \\
C4A & $0.0160(6)$ & $0.0180(6)$ & $0.0156(6)$ & $-0.0034(5)$ & $-0.0003(5)$ & $0.0008(5)$ \\
C5 & $0.0200(7)$ & $0.0199(6)$ & $0.0233(7)$ & $-0.0036(5)$ & $-0.0003(5)$ & $-0.0028(5)$ \\
C6 & $0.0212(7)$ & $0.0262(7)$ & $0.0274(7)$ & $0.0023(6)$ & $-0.0001(6)$ & $-0.0064(6)$ \\
C7 & $0.0161(7)$ & $0.0326(8)$ & $0.0295(8)$ & $-0.0016(6)$ & $0.0020(6)$ & $-0.0028(6)$ \\
C8 & $0.0159(7)$ & $0.0265(7)$ & $0.0251(7)$ & $-0.0070(5)$ & $0.0008(5)$ & $-0.0011(6)$
\end{tabular}


supporting information

\begin{tabular}{lllllll} 
C8A & $0.0155(6)$ & $0.0195(6)$ & $0.0167(6)$ & $-0.0045(5)$ & $0.0007(5)$ & $0.0012(5)$ \\
C21 & $0.0246(8)$ & $0.0188(7)$ & $0.0374(8)$ & $-0.0057(6)$ & $0.0054(6)$ & $-0.0077(6)$ \\
C31 & $0.0179(6)$ & $0.0169(6)$ & $0.0194(6)$ & $-0.0048(5)$ & $0.0019(5)$ & $-0.0020(5)$ \\
O31 & $0.0167(5)$ & $0.0347(6)$ & $0.0231(5)$ & $-0.0001(4)$ & $-0.0004(4)$ & $0.0009(4)$ \\
O32 & $0.0202(5)$ & $0.0344(6)$ & $0.0178(5)$ & $-0.0032(4)$ & $0.0034(4)$ & $-0.0043(4)$ \\
C32 & $0.0250(8)$ & $0.0413(9)$ & $0.0258(8)$ & $-0.0002(7)$ & $0.0109(6)$ & $-0.0066(7)$ \\
C33 & $0.0262(8)$ & $0.0466(10)$ & $0.0293(8)$ & $-0.0023(7)$ & $0.0084(7)$ & $0.0050(7)$ \\
C41 & $0.0153(6)$ & $0.0167(6)$ & $0.0184(6)$ & $-0.0038(5)$ & $-0.0019(5)$ & $-0.0004(5)$ \\
C42 & $0.0202(7)$ & $0.0185(6)$ & $0.0165(6)$ & $-0.0070(5)$ & $-0.0027(5)$ & $-0.0003(5)$ \\
C421 & $0.0171(6)$ & $0.0174(6)$ & $0.0175(6)$ & $-0.0054(5)$ & $-0.0013(5)$ & $0.0010(5)$ \\
C422 & $0.0201(7)$ & $0.0206(6)$ & $0.0162(6)$ & $-0.0066(5)$ & $0.0028(5)$ & $-0.0002(5)$ \\
C423 & $0.0191(7)$ & $0.0194(6)$ & $0.0179(6)$ & $-0.0068(5)$ & $0.0011(5)$ & $-0.0029(5)$ \\
C424 & $0.0160(6)$ & $0.0158(6)$ & $0.0211(6)$ & $-0.0069(5)$ & $0.0003(5)$ & $0.0003(5)$ \\
Br42 & $0.02136(8)$ & $0.02079(8)$ & $0.02118(7)$ & $-0.01179(5)$ & $0.00231(5)$ & $-0.00103(5)$ \\
C425 & $0.0268(8)$ & $0.0264(7)$ & $0.0174(6)$ & $-0.0125(6)$ & $0.0020(6)$ & $0.0032(5)$ \\
C426 & $0.0286(8)$ & $0.0261(7)$ & $0.0158(6)$ & $-0.0132(6)$ & $-0.0015(5)$ & $-0.0004(5)$ \\
& & & & & \\
\hline
\end{tabular}

Geometric parameters $\left(\stackrel{A}{A}{ }^{\circ}\right)$

\begin{tabular}{llll}
\hline $\mathrm{N} 1-\mathrm{C} 2$ & $1.3160(18)$ & $\mathrm{O} 32-\mathrm{C} 32$ & $1.4638(17)$ \\
$\mathrm{N} 1-\mathrm{C} 8 \mathrm{~A}$ & $1.3725(18)$ & $\mathrm{C} 32-\mathrm{C} 33$ & $1.504(2)$ \\
$\mathrm{C} 2-\mathrm{C} 3$ & $1.4282(18)$ & $\mathrm{C} 32-\mathrm{H} 32 \mathrm{~A}$ & 0.9900 \\
$\mathrm{C} 2-\mathrm{C} 21$ & $1.5038(19)$ & $\mathrm{C} 32-\mathrm{H} 32 \mathrm{~B}$ & 0.9900 \\
$\mathrm{C} 3-\mathrm{C} 4$ & $1.3784(19)$ & $\mathrm{C} 33-\mathrm{H} 33 \mathrm{~A}$ & 0.9800 \\
$\mathrm{C} 3-\mathrm{C} 31$ & $1.5042(19)$ & $\mathrm{C} 33-\mathrm{H} 33 \mathrm{~B}$ & 0.9800 \\
$\mathrm{C} 4-\mathrm{C} 4 \mathrm{~A}$ & $1.4309(18)$ & $\mathrm{C} 33-\mathrm{H} 33 \mathrm{C}$ & 0.9800 \\
$\mathrm{C} 4-\mathrm{C} 41$ & $1.4741(18)$ & $\mathrm{C} 41-\mathrm{C} 42$ & $1.3341(19)$ \\
$\mathrm{C} 4 \mathrm{~A}-\mathrm{C} 8 \mathrm{~A}$ & $1.4168(18)$ & $\mathrm{C} 41-\mathrm{H} 41$ & 0.9500 \\
$\mathrm{C} 4 \mathrm{~A}-\mathrm{C} 5$ & $1.4173(19)$ & $\mathrm{C} 42-\mathrm{C} 421$ & $1.4676(18)$ \\
$\mathrm{C} 5-\mathrm{C} 6$ & $1.370(2)$ & $\mathrm{C} 42-\mathrm{H} 42$ & 0.9500 \\
$\mathrm{C} 5-\mathrm{H} 5$ & 0.9500 & $\mathrm{C} 421-\mathrm{C} 426$ & $1.3942(19)$ \\
$\mathrm{C} 6-\mathrm{C} 7$ & $1.406(2)$ & $\mathrm{C} 421-\mathrm{C} 422$ & $1.3977(18)$ \\
$\mathrm{C} 6-\mathrm{H} 6$ & 0.9500 & $\mathrm{C} 422-\mathrm{C} 423$ & $1.3834(18)$ \\
$\mathrm{C} 7-\mathrm{C} 8$ & $1.368(2)$ & $\mathrm{C} 422-\mathrm{H} 422$ & 0.9500 \\
$\mathrm{C} 7-\mathrm{H} 7$ & 0.9500 & $\mathrm{C} 423-\mathrm{C} 424$ & $1.3891(18)$ \\
$\mathrm{C} 8-\mathrm{C} 8 \mathrm{~A}$ & $1.4152(19)$ & $\mathrm{C} 423-\mathrm{H} 423$ & 0.9500 \\
$\mathrm{C} 8-\mathrm{H} 8$ & 0.9500 & $\mathrm{C} 424-\mathrm{C} 425$ & $1.3820(19)$ \\
$\mathrm{C} 21-\mathrm{H} 21 \mathrm{~A}$ & 0.9800 & $\mathrm{C} 424-\mathrm{B} 42$ & $1.8996(13)$ \\
$\mathrm{C} 21-\mathrm{H} 21 \mathrm{~B}$ & 0.9800 & $\mathrm{C} 425-\mathrm{C} 426$ & $1.3889(19)$ \\
$\mathrm{C} 21-\mathrm{H} 21 \mathrm{C}$ & 0.9800 & $\mathrm{C} 425-\mathrm{H} 425$ & 0.9500 \\
$\mathrm{C} 31-\mathrm{O} 31$ & $1.1997(17)$ & $\mathrm{C} 426-\mathrm{H} 426$ & 0.9500 \\
$\mathrm{C} 31-\mathrm{O} 32$ & $1.3409(17)$ & & 109.5 \\
$\mathrm{C} 2-\mathrm{N} 1-\mathrm{C} 8 \mathrm{~A}$ & $118.72(12)$ & $\mathrm{C} 31-\mathrm{O} 32-\mathrm{C} 32$ & $116.51(12)$ \\
$\mathrm{N} 1-\mathrm{C} 2-\mathrm{C} 3$ & $122.30(13)$ & $\mathrm{O} 32-\mathrm{C} 32-\mathrm{C} 33$ & $109.54(13)$ \\
$\mathrm{N} 1-\mathrm{C} 2-\mathrm{C} 21$ & $117.24(12)$ & $\mathrm{O} 32-\mathrm{C} 32-\mathrm{H} 32 \mathrm{~A}$ & \\
$\mathrm{C} 3-\mathrm{C} 2-\mathrm{C} 21$ & $120.46(12)$ & $\mathrm{C} 33-\mathrm{C} 32-\mathrm{H} 32 \mathrm{~A}$ & \\
$\mathrm{C} 4-\mathrm{C} 3-\mathrm{C} 2$ & $120.41(12)$ & $\mathrm{O} 32-\mathrm{C} 32-\mathrm{H} 32 \mathrm{~B}$ & \\
& & &
\end{tabular}




\begin{tabular}{|c|c|c|c|}
\hline $\mathrm{C} 4-\mathrm{C} 3-\mathrm{C} 31$ & $121.32(12)$ & $\mathrm{C} 33-\mathrm{C} 32-\mathrm{H} 32 \mathrm{~B}$ & 109.5 \\
\hline $\mathrm{C} 2-\mathrm{C} 3-\mathrm{C} 31$ & $118.24(12)$ & $\mathrm{H} 32 \mathrm{~A}-\mathrm{C} 32-\mathrm{H} 32 \mathrm{~B}$ & 108.1 \\
\hline $\mathrm{C} 3-\mathrm{C} 4-\mathrm{C} 4 \mathrm{~A}$ & $117.88(12)$ & C32-C33-H33A & 109.5 \\
\hline $\mathrm{C} 3-\mathrm{C} 4-\mathrm{C} 41$ & $122.47(12)$ & C $32-\mathrm{C} 33-\mathrm{H} 33 \mathrm{~B}$ & 109.5 \\
\hline $\mathrm{C} 4 \mathrm{~A}-\mathrm{C} 4-\mathrm{C} 41$ & $119.64(12)$ & $\mathrm{H} 33 \mathrm{~A}-\mathrm{C} 33-\mathrm{H} 33 \mathrm{~B}$ & 109.5 \\
\hline $\mathrm{C} 8 \mathrm{~A}-\mathrm{C} 4 \mathrm{~A}-\mathrm{C} 5$ & $118.96(12)$ & $\mathrm{C} 32-\mathrm{C} 33-\mathrm{H} 33 \mathrm{C}$ & 109.5 \\
\hline $\mathrm{C} 8 \mathrm{~A}-\mathrm{C} 4 \mathrm{~A}-\mathrm{C} 4$ & $117.89(12)$ & $\mathrm{H} 33 \mathrm{~A}-\mathrm{C} 33-\mathrm{H} 33 \mathrm{C}$ & 109.5 \\
\hline $\mathrm{C} 5-\mathrm{C} 4 \mathrm{~A}-\mathrm{C} 4$ & $123.04(12)$ & $\mathrm{H} 33 \mathrm{~B}-\mathrm{C} 33-\mathrm{H} 33 \mathrm{C}$ & 109.5 \\
\hline $\mathrm{C} 6-\mathrm{C} 5-\mathrm{C} 4 \mathrm{~A}$ & $120.45(13)$ & $\mathrm{C} 42-\mathrm{C} 41-\mathrm{C} 4$ & $123.28(12)$ \\
\hline $\mathrm{C} 6-\mathrm{C} 5-\mathrm{H} 5$ & 119.8 & $\mathrm{C} 42-\mathrm{C} 41-\mathrm{H} 41$ & 118.4 \\
\hline $\mathrm{C} 4 \mathrm{~A}-\mathrm{C} 5-\mathrm{H} 5$ & 119.8 & $\mathrm{C} 4-\mathrm{C} 41-\mathrm{H} 41$ & 118.4 \\
\hline $\mathrm{C} 5-\mathrm{C} 6-\mathrm{C} 7$ & $120.38(14)$ & $\mathrm{C} 41-\mathrm{C} 42-\mathrm{C} 421$ & $126.00(13)$ \\
\hline $\mathrm{C} 5-\mathrm{C} 6-\mathrm{H} 6$ & 119.8 & $\mathrm{C} 41-\mathrm{C} 42-\mathrm{H} 42$ & 117.0 \\
\hline $\mathrm{C} 7-\mathrm{C} 6-\mathrm{H} 6$ & 119.8 & $\mathrm{C} 421-\mathrm{C} 42-\mathrm{H} 42$ & 117.0 \\
\hline $\mathrm{C} 8-\mathrm{C} 7-\mathrm{C} 6$ & $120.58(14)$ & $\mathrm{C} 426-\mathrm{C} 421-\mathrm{C} 422$ & $118.37(12)$ \\
\hline $\mathrm{C} 8-\mathrm{C} 7-\mathrm{H} 7$ & 119.7 & $\mathrm{C} 426-\mathrm{C} 421-\mathrm{C} 42$ & $118.82(12)$ \\
\hline $\mathrm{C} 6-\mathrm{C} 7-\mathrm{H} 7$ & 119.7 & $\mathrm{C} 422-\mathrm{C} 421-\mathrm{C} 42$ & $122.80(12)$ \\
\hline $\mathrm{C} 7-\mathrm{C} 8-\mathrm{C} 8 \mathrm{~A}$ & $120.35(13)$ & $\mathrm{C} 423-\mathrm{C} 422-\mathrm{C} 421$ & $120.74(12)$ \\
\hline $\mathrm{C} 7-\mathrm{C} 8-\mathrm{H} 8$ & 119.8 & $\mathrm{C} 423-\mathrm{C} 422-\mathrm{H} 422$ & 119.6 \\
\hline $\mathrm{C} 8 \mathrm{~A}-\mathrm{C} 8-\mathrm{H} 8$ & 119.8 & $\mathrm{C} 421-\mathrm{C} 422-\mathrm{H} 422$ & 119.6 \\
\hline $\mathrm{N} 1-\mathrm{C} 8 \mathrm{~A}-\mathrm{C} 8$ & $118.03(12)$ & $\mathrm{C} 422-\mathrm{C} 423-\mathrm{C} 424$ & $119.36(12)$ \\
\hline $\mathrm{N} 1-\mathrm{C} 8 \mathrm{~A}-\mathrm{C} 4 \mathrm{~A}$ & $122.69(12)$ & $\mathrm{C} 422-\mathrm{C} 423-\mathrm{H} 423$ & 120.3 \\
\hline $\mathrm{C} 8-\mathrm{C} 8 \mathrm{~A}-\mathrm{C} 4 \mathrm{~A}$ & $119.27(13)$ & $\mathrm{C} 424-\mathrm{C} 423-\mathrm{H} 423$ & 120.3 \\
\hline $\mathrm{C} 2-\mathrm{C} 21-\mathrm{H} 21 \mathrm{~A}$ & 109.5 & $\mathrm{C} 425-\mathrm{C} 424-\mathrm{C} 423$ & $121.43(12)$ \\
\hline $\mathrm{C} 2-\mathrm{C} 21-\mathrm{H} 21 \mathrm{~B}$ & 109.5 & $\mathrm{C} 425-\mathrm{C} 424-\mathrm{Br} 42$ & $119.86(10)$ \\
\hline $\mathrm{H} 21 \mathrm{~A}-\mathrm{C} 21-\mathrm{H} 21 \mathrm{~B}$ & 109.5 & $\mathrm{C} 423-\mathrm{C} 424-\mathrm{Br} 42$ & $118.69(10)$ \\
\hline $\mathrm{C} 2-\mathrm{C} 21-\mathrm{H} 21 \mathrm{C}$ & 109.5 & $\mathrm{C} 424-\mathrm{C} 425-\mathrm{C} 426$ & $118.42(13)$ \\
\hline $\mathrm{H} 21 \mathrm{~A}-\mathrm{C} 21-\mathrm{H} 21 \mathrm{C}$ & 109.5 & $\mathrm{C} 424-\mathrm{C} 425-\mathrm{H} 425$ & 120.8 \\
\hline $\mathrm{H} 21 \mathrm{~B}-\mathrm{C} 21-\mathrm{H} 21 \mathrm{C}$ & 109.5 & $\mathrm{C} 426-\mathrm{C} 425-\mathrm{H} 425$ & 120.8 \\
\hline $\mathrm{O} 31-\mathrm{C} 31-\mathrm{O} 32$ & $124.62(13)$ & $\mathrm{C} 425-\mathrm{C} 426-\mathrm{C} 421$ & $121.67(13)$ \\
\hline $\mathrm{O} 31-\mathrm{C} 31-\mathrm{C} 3$ & $124.83(12)$ & $\mathrm{C} 425-\mathrm{C} 426-\mathrm{H} 426$ & 119.2 \\
\hline $\mathrm{O} 32-\mathrm{C} 31-\mathrm{C} 3$ & $110.53(12)$ & $\mathrm{C} 421-\mathrm{C} 426-\mathrm{H} 426$ & 119.2 \\
\hline $\mathrm{C} 8 \mathrm{~A}-\mathrm{N} 1-\mathrm{C} 2-\mathrm{C} 3$ & $-0.3(2)$ & $\mathrm{C} 5-\mathrm{C} 4 \mathrm{~A}-\mathrm{C} 8 \mathrm{~A}-\mathrm{C} 8$ & $0.6(2)$ \\
\hline $\mathrm{C} 8 \mathrm{~A}-\mathrm{N} 1-\mathrm{C} 2-\mathrm{C} 21$ & $179.82(13)$ & $\mathrm{C} 4-\mathrm{C} 4 \mathrm{~A}-\mathrm{C} 8 \mathrm{~A}-\mathrm{C} 8$ & $-175.68(12)$ \\
\hline $\mathrm{N} 1-\mathrm{C} 2-\mathrm{C} 3-\mathrm{C} 4$ & $2.6(2)$ & $\mathrm{C} 4-\mathrm{C} 3-\mathrm{C} 31-\mathrm{O} 31$ & $75.39(19)$ \\
\hline $\mathrm{C} 21-\mathrm{C} 2-\mathrm{C} 3-\mathrm{C} 4$ & $-177.51(13)$ & $\mathrm{C} 2-\mathrm{C} 3-\mathrm{C} 31-\mathrm{O} 31$ & $-102.56(17)$ \\
\hline $\mathrm{N} 1-\mathrm{C} 2-\mathrm{C} 3-\mathrm{C} 31$ & $-179.45(12)$ & $\mathrm{C} 4-\mathrm{C} 3-\mathrm{C} 31-\mathrm{O} 32$ & $-106.17(14)$ \\
\hline $\mathrm{C} 21-\mathrm{C} 2-\mathrm{C} 3-\mathrm{C} 31$ & $0.46(19)$ & $\mathrm{C} 2-\mathrm{C} 3-\mathrm{C} 31-\mathrm{O} 32$ & $75.88(15)$ \\
\hline $\mathrm{C} 2-\mathrm{C} 3-\mathrm{C} 4-\mathrm{C} 4 \mathrm{~A}$ & $-1.82(19)$ & $\mathrm{O} 31-\mathrm{C} 31-\mathrm{O} 32-\mathrm{C} 32$ & $-1.2(2)$ \\
\hline $\mathrm{C} 31-\mathrm{C} 3-\mathrm{C} 4-\mathrm{C} 4 \mathrm{~A}$ & $-179.73(12)$ & $\mathrm{C} 3-\mathrm{C} 31-\mathrm{O} 32-\mathrm{C} 32$ & $-179.65(12)$ \\
\hline $\mathrm{C} 2-\mathrm{C} 3-\mathrm{C} 4-\mathrm{C} 41$ & $179.17(12)$ & $\mathrm{C} 31-\mathrm{O} 32-\mathrm{C} 32-\mathrm{C} 33$ & $-85.21(17)$ \\
\hline $\mathrm{C} 31-\mathrm{C} 3-\mathrm{C} 4-\mathrm{C} 41$ & $1.3(2)$ & $\mathrm{C} 3-\mathrm{C} 4-\mathrm{C} 41-\mathrm{C} 42$ & $51.4(2)$ \\
\hline $\mathrm{C} 3-\mathrm{C} 4-\mathrm{C} 4 \mathrm{~A}-\mathrm{C} 8 \mathrm{~A}$ & $-0.97(18)$ & $\mathrm{C} 4 \mathrm{~A}-\mathrm{C} 4-\mathrm{C} 41-\mathrm{C} 42$ & $-127.57(15)$ \\
\hline $\mathrm{C} 41-\mathrm{C} 4-\mathrm{C} 4 \mathrm{~A}-\mathrm{C} 8 \mathrm{~A}$ & $178.07(12)$ & $\mathrm{C} 4-\mathrm{C} 41-\mathrm{C} 42-\mathrm{C} 421$ & $-178.74(13)$ \\
\hline $\mathrm{C} 3-\mathrm{C} 4-\mathrm{C} 4 \mathrm{~A}-\mathrm{C} 5$ & $-177.12(13)$ & $\mathrm{C} 41-\mathrm{C} 42-\mathrm{C} 421-\mathrm{C} 426$ & $-170.67(15)$ \\
\hline $\mathrm{C} 41-\mathrm{C} 4-\mathrm{C} 4 \mathrm{~A}-\mathrm{C} 5$ & $1.9(2)$ & $\mathrm{C} 41-\mathrm{C} 42-\mathrm{C} 421-\mathrm{C} 422$ & $10.6(2)$ \\
\hline $\mathrm{C} 8 \mathrm{~A}-\mathrm{C} 4 \mathrm{~A}-\mathrm{C} 5-\mathrm{C} 6$ & $-0.9(2)$ & $\mathrm{C} 426-\mathrm{C} 421-\mathrm{C} 422-\mathrm{C} 423$ & $0.4(2)$ \\
\hline
\end{tabular}




$\begin{array}{ll}\mathrm{C} 4-\mathrm{C} 4 \mathrm{~A}-\mathrm{C} 5-\mathrm{C} 6 & 175.18(13) \\ \mathrm{C} 4 \mathrm{~A}-\mathrm{C} 5-\mathrm{C} 6-\mathrm{C} 7 & 0.4(2) \\ \mathrm{C} 5-\mathrm{C} 6-\mathrm{C} 7-\mathrm{C} 8 & 0.5(2) \\ \mathrm{C} 6-\mathrm{C} 7-\mathrm{C} 8-\mathrm{C} 8 \mathrm{~A} & -0.8(2) \\ \mathrm{C} 2-\mathrm{N} 1-\mathrm{C} 8 \mathrm{~A}-\mathrm{C} 8 & 176.31(13) \\ \mathrm{C} 2-\mathrm{N} 1-\mathrm{C} 8 \mathrm{~A}-\mathrm{C} 4 \mathrm{~A} & -2.7(2) \\ \mathrm{C} 7-\mathrm{C} 8-\mathrm{C} 8 \mathrm{~A}-\mathrm{N} 1 & -178.88(14) \\ \mathrm{C} 7-\mathrm{C} 8-\mathrm{C} 8 \mathrm{~A}-\mathrm{C} 4 \mathrm{~A} & 0.2(2) \\ \mathrm{C} 5-\mathrm{C} 4 \mathrm{~A}-\mathrm{C} 8 \mathrm{~A}-\mathrm{N} 1 & 179.67(13) \\ \mathrm{C} 4-\mathrm{C} 4 \mathrm{~A}-\mathrm{C} 8 \mathrm{~A}-\mathrm{N} 1 & 3.4(2)\end{array}$

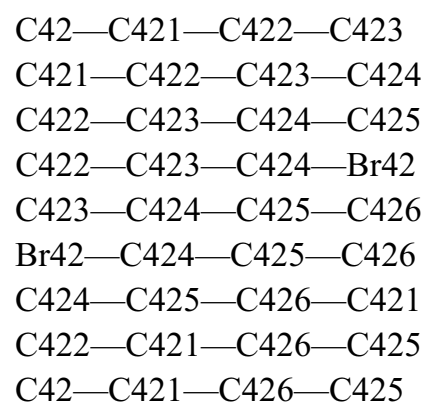

$179.12(13)$

$0.3(2)$

$-178.17(11)$

$-0.7(2)$

$177.77(12)$

0.9 (2)

$-0.8(2)$

$-179.57(14)$

Hydrogen-bond geometry $\left(\AA,{ }^{\circ}\right)$

\begin{tabular}{lllll}
\hline$D-\mathrm{H} \cdots A$ & $D-\mathrm{H}$ & $\mathrm{H} \cdots A$ & $D \cdots A$ & $D-\mathrm{H} \cdots A$ \\
\hline $\mathrm{C} 423-\mathrm{H} 423 \cdots \mathrm{O} 31^{\mathrm{i}}$ & 0.95 & 2.59 & $3.2197(17)$ & 124 \\
$\mathrm{C} 426-\mathrm{H} 426 \cdots C g 1^{\mathrm{ii}}$ & 0.95 & 2.74 & $3.5961(16)$ & 151 \\
\hline
\end{tabular}

Symmetry codes: (i) $-x+3 / 2, y+1 / 2,-z+3 / 2$; (ii) $-x+1,-y+1,-z+1$.

Ethyl (E)-2-methyl-4-\{2-[4-(trifluoromethyl)phenyl] ethenyl\}quinoline-3-carboxylate (VI)

\section{Crystal data}

$\mathrm{C}_{22} \mathrm{H}_{18} \mathrm{~F}_{3} \mathrm{NO}_{2}$

$M_{r}=385.37$

Triclinic, $P \overline{1}$

$a=8.7465(10) \AA$

$b=9.9436(11) \AA$

$c=11.1116(11) \AA$

$\alpha=105.446(4)^{\circ}$

$\beta=99.763(4)^{\circ}$

$\gamma=97.204(4)^{\circ}$

$V=903.08(17) \AA^{3}$

\section{Data collection}

Bruker D8 Venture diffractometer

Radiation source: INCOATEC high brilliance microfocus sealed tube

Multilayer mirror monochromator $\varphi$ and $\omega$ scans

Absorption correction: multi-scan

(SADABS; Bruker, 2016)

$T_{\min }=0.954, T_{\max }=0.980$

\section{Refinement}

Refinement on $F^{2}$

Least-squares matrix: full

$R\left[F^{2}>2 \sigma\left(F^{2}\right)\right]=0.044$

$w R\left(F^{2}\right)=0.120$

$S=1.05$

4491 reflections

255 parameters

0 restraints
$Z=2$

$F(000)=400$

$D_{\mathrm{x}}=1.417 \mathrm{Mg} \mathrm{m}^{-3}$

Mo $K \alpha$ radiation, $\lambda=0.71073 \AA$

Cell parameters from 4022 reflections

$\theta=2.2-28.3^{\circ}$

$\mu=0.11 \mathrm{~mm}^{-1}$

$T=100 \mathrm{~K}$

Block, yellow

$0.27 \times 0.20 \times 0.18 \mathrm{~mm}$

59876 measured reflections

4491 independent reflections

3692 reflections with $I>2 \sigma(I)$

$R_{\text {int }}=0.042$

$\theta_{\max }=28.3^{\circ}, \theta_{\min }=2.2^{\circ}$

$h=-11 \rightarrow 11$

$k=-13 \rightarrow 13$

$l=-14 \rightarrow 14$

Primary atom site location: difference Fourier map

Hydrogen site location: inferred from neighbouring sites

$\mathrm{H}$-atom parameters constrained

$w=1 /\left[\sigma^{2}\left(F_{\mathrm{o}}^{2}\right)+(0.0452 P)^{2}+0.7003 P\right]$

where $P=\left(F_{\mathrm{o}}^{2}+2 F_{\mathrm{c}}^{2}\right) / 3$

$(\Delta / \sigma)_{\max }<0.001$ 
$\Delta \rho_{\max }=0.43$ e $\AA^{-3}$

$\Delta \rho_{\min }=-0.43$ e $\AA^{-3}$

Special details

Geometry. All esds (except the esd in the dihedral angle between two 1.s. planes) are estimated using the full covariance matrix. The cell esds are taken into account individually in the estimation of esds in distances, angles and torsion angles; correlations between esds in cell parameters are only used when they are defined by crystal symmetry. An approximate (isotropic) treatment of cell esds is used for estimating esds involving l.s. planes.

Fractional atomic coordinates and isotropic or equivalent isotropic displacement parameters $\left(\AA^{2}\right)$

\begin{tabular}{|c|c|c|c|c|}
\hline & $x$ & $y$ & $z$ & $U_{\text {iso }} * / U_{\text {eq }}$ \\
\hline N1 & 0.07789 (14) & $0.22664(13)$ & $0.15945(11)$ & $0.0204(2)$ \\
\hline $\mathrm{C} 2$ & $0.07976(16)$ & $0.36156(15)$ & $0.21549(13)$ & $0.0192(3)$ \\
\hline $\mathrm{C} 3$ & $0.16984(15)$ & $0.43512(14)$ & $0.34110(13)$ & $0.0171(3)$ \\
\hline $\mathrm{C} 4$ & $0.25843(15)$ & $0.36523(14)$ & $0.41074(12)$ & $0.0168(3)$ \\
\hline $\mathrm{C} 4 \mathrm{~A}$ & $0.26008(15)$ & $0.21910(14)$ & $0.35031(13)$ & $0.0173(3)$ \\
\hline $\mathrm{C} 5$ & $0.34837(17)$ & $0.13520(15)$ & $0.40993(14)$ & $0.0216(3)$ \\
\hline H5 & 0.4093 & 0.1758 & 0.4944 & $0.026^{*}$ \\
\hline C6 & $0.34646(18)$ & $-0.00371(16)$ & $0.34678(15)$ & $0.0251(3)$ \\
\hline H6 & 0.4067 & -0.0585 & 0.3877 & $0.030 *$ \\
\hline $\mathrm{C} 7$ & $0.25608(19)$ & $-0.06624(15)$ & $0.22177(14)$ & $0.0250(3)$ \\
\hline H7 & 0.2562 & -0.1626 & 0.1789 & $0.030 *$ \\
\hline $\mathrm{C} 8$ & $0.16816(18)$ & $0.01086(15)$ & $0.16161(14)$ & $0.0232(3)$ \\
\hline $\mathrm{H} 8$ & 0.1067 & -0.0323 & 0.0776 & $0.028 *$ \\
\hline $\mathrm{C} 8 \mathrm{~A}$ & $0.16872(16)$ & $0.15504(14)$ & $0.22455(13)$ & $0.0187(3)$ \\
\hline $\mathrm{C} 21$ & $-0.01256(19)$ & $0.43927(16)$ & $0.13843(14)$ & $0.0251(3)$ \\
\hline $\mathrm{H} 21 \mathrm{~A}$ & -0.0605 & 0.3749 & 0.0530 & $0.038 *$ \\
\hline $\mathrm{H} 21 \mathrm{~B}$ & 0.0581 & 0.5199 & 0.1310 & $0.038^{*}$ \\
\hline $\mathrm{H} 21 \mathrm{C}$ & -0.0955 & 0.4737 & 0.1810 & $0.038^{*}$ \\
\hline C31 & $0.17896(16)$ & $0.59297(14)$ & $0.38398(13)$ & $0.0180(3)$ \\
\hline $\mathrm{O} 31$ & $0.29053(12)$ & $0.67679(11)$ & $0.38197(10)$ & $0.0231(2)$ \\
\hline $\mathrm{O} 32$ & $0.04628(11)$ & $0.62833(10)$ & $0.41672(9)$ & $0.0200(2)$ \\
\hline $\mathrm{C} 32$ & $0.03379(17)$ & $0.77805(14)$ & $0.44196(14)$ & $0.0207(3)$ \\
\hline $\mathrm{H} 32 \mathrm{~A}$ & -0.0788 & 0.7871 & 0.4320 & $0.025 *$ \\
\hline H32B & 0.0773 & 0.8161 & 0.3786 & $0.025^{*}$ \\
\hline C33 & $0.12155(18)$ & $0.86413(16)$ & $0.57495(14)$ & $0.0250(3)$ \\
\hline H33A & 0.0848 & 0.8219 & 0.6375 & $0.037 *$ \\
\hline H33B & 0.1019 & 0.9616 & 0.5917 & $0.037^{*}$ \\
\hline $\mathrm{H} 33 \mathrm{C}$ & 0.2348 & 0.8646 & 0.5818 & $0.037 *$ \\
\hline $\mathrm{C} 41$ & $0.35128(16)$ & $0.43372(14)$ & $0.54207(13)$ & $0.0184(3)$ \\
\hline $\mathrm{H} 41$ & 0.4482 & 0.4038 & 0.5650 & $0.022 *$ \\
\hline $\mathrm{C} 42$ & $0.31086(16)$ & $0.53430(15)$ & $0.63157(13)$ & $0.0193(3)$ \\
\hline $\mathrm{H} 42$ & 0.2140 & 0.5648 & 0.6098 & $0.023 *$ \\
\hline $\mathrm{C} 421$ & $0.40671(16)$ & $0.60055(15)$ & $0.76133(13)$ & $0.0192(3)$ \\
\hline $\mathrm{C} 422$ & $0.52605(17)$ & $0.53948(15)$ & $0.81430(13)$ & $0.0213(3)$ \\
\hline $\mathrm{H} 422$ & 0.5451 & 0.4509 & 0.7667 & $0.026^{*}$ \\
\hline $\mathrm{C} 423$ & $0.61718(17)$ & $0.60661(16)$ & $0.93570(14)$ & $0.0236(3)$ \\
\hline $\mathrm{H} 423$ & 0.6985 & 0.5643 & 0.9707 & $0.028 *$ \\
\hline $\mathrm{C} 424$ & $0.58945(17)$ & $0.73547(16)$ & $1.00582(13)$ & $0.0228(3)$ \\
\hline
\end{tabular}


supporting information

\begin{tabular}{lllll} 
C425 & $0.46796(19)$ & $0.79605(16)$ & $0.95666(14)$ & $0.0259(3)$ \\
H425 & 0.4474 & 0.8832 & 1.0059 & $0.031^{*}$ \\
C426 & $0.37692(18)$ & $0.72844(16)$ & $0.83523(14)$ & $0.0240(3)$ \\
H426 & 0.2933 & 0.7695 & 0.8018 & $0.029^{*}$ \\
C427 & $0.6902(2)$ & $0.81210(18)$ & $1.13510(15)$ & $0.0302(3)$ \\
F471 & $0.74662(16)$ & $0.94629(12)$ & $1.14494(11)$ & $0.0523(3)$ \\
F472 & $0.81434(15)$ & $0.75355(14)$ & $1.16419(11)$ & $0.0539(4)$ \\
F473 & $0.61164(15)$ & $0.81641(14)$ & $1.22927(10)$ & $0.0506(3)$ \\
\hline
\end{tabular}

Atomic displacement parameters $\left(\AA^{2}\right)$

\begin{tabular}{|c|c|c|c|c|c|c|}
\hline & $U^{11}$ & $U^{22}$ & $U^{33}$ & $U^{12}$ & $U^{13}$ & $U^{23}$ \\
\hline N1 & $0.0231(6)$ & $0.0206(6)$ & 0.0159 (5) & 0.0032 (4) & 0.0012 (4) & 0.0049 (4) \\
\hline $\mathrm{C} 2$ & $0.0200(6)$ & $0.0198(6)$ & $0.0175(6)$ & $0.0032(5)$ & $0.0025(5)$ & $0.0061(5)$ \\
\hline $\mathrm{C} 3$ & $0.0170(6)$ & $0.0167(6)$ & $0.0178(6)$ & $0.0030(5)$ & $0.0044(5)$ & $0.0050(5)$ \\
\hline $\mathrm{C} 4$ & $0.0153(6)$ & $0.0176(6)$ & $0.0164(6)$ & $0.0014(5)$ & $0.0026(5)$ & $0.0045(5)$ \\
\hline $\mathrm{C} 4 \mathrm{~A}$ & $0.0178(6)$ & $0.0166(6)$ & $0.0177(6)$ & $0.0027(5)$ & $0.0037(5)$ & $0.0054(5)$ \\
\hline C5 & $0.0227(7)$ & $0.0206(7)$ & $0.0203(6)$ & 0.0039 (5) & 0.0015 (5) & $0.0061(5)$ \\
\hline C6 & $0.0291(7)$ & $0.0198(7)$ & $0.0263(7)$ & $0.0070(6)$ & $0.0018(6)$ & 0.0079 (6) \\
\hline $\mathrm{C} 7$ & $0.0311(8)$ & $0.0170(6)$ & $0.0250(7)$ & $0.0051(6)$ & $0.0050(6)$ & $0.0036(5)$ \\
\hline $\mathrm{C} 8$ & $0.0286(7)$ & $0.0201(7)$ & $0.0181(6)$ & $0.0030(5)$ & $0.0032(5)$ & $0.0023(5)$ \\
\hline $\mathrm{C} 8 \mathrm{~A}$ & $0.0201(6)$ & $0.0187(6)$ & 0.0169 (6) & $0.0028(5)$ & $0.0034(5)$ & $0.0053(5)$ \\
\hline $\mathrm{C} 21$ & $0.0292(7)$ & $0.0249(7)$ & $0.0196(7)$ & $0.0059(6)$ & $-0.0017(5)$ & $0.0080(5)$ \\
\hline $\mathrm{C} 31$ & $0.0192(6)$ & $0.0183(6)$ & $0.0166(6)$ & $0.0044(5)$ & $0.0024(5)$ & $0.0055(5)$ \\
\hline $\mathrm{O} 31$ & $0.0212(5)$ & $0.0190(5)$ & $0.0293(5)$ & 0.0034 (4) & 0.0061 (4) & 0.0070 (4) \\
\hline $\mathrm{O} 32$ & $0.0183(5)$ & $0.0184(5)$ & $0.0230(5)$ & 0.0045 (4) & 0.0043 (4) & 0.0051 (4) \\
\hline $\mathrm{C} 32$ & $0.0216(6)$ & $0.0184(6)$ & $0.0225(7)$ & $0.0072(5)$ & $0.0035(5)$ & 0.0057 (5) \\
\hline $\mathrm{C} 33$ & $0.0271(7)$ & $0.0217(7)$ & $0.0236(7)$ & $0.0065(6)$ & $0.0028(6)$ & $0.0032(5)$ \\
\hline C41 & $0.0165(6)$ & $0.0186(6)$ & $0.0182(6)$ & $0.0005(5)$ & $0.0004(5)$ & $0.0055(5)$ \\
\hline C42 & $0.0181(6)$ & $0.0204(6)$ & $0.0177(6)$ & $0.0023(5)$ & $0.0011(5)$ & $0.0050(5)$ \\
\hline $\mathrm{C} 421$ & $0.0183(6)$ & $0.0207(6)$ & $0.0167(6)$ & $0.0015(5)$ & $0.0026(5)$ & $0.0038(5)$ \\
\hline $\mathrm{C} 422$ & $0.0215(7)$ & $0.0219(7)$ & $0.0191(6)$ & $0.0050(5)$ & $0.0034(5)$ & $0.0037(5)$ \\
\hline $\mathrm{C} 423$ & $0.0220(7)$ & $0.0288(7)$ & $0.0196(7)$ & $0.0068(6)$ & $0.0018(5)$ & $0.0069(6)$ \\
\hline C424 & $0.0234(7)$ & $0.0246(7)$ & $0.0164(6)$ & 0.0007 (5) & $0.0013(5)$ & $0.0027(5)$ \\
\hline $\mathrm{C} 425$ & $0.0307(8)$ & $0.0207(7)$ & $0.0214(7)$ & $0.0047(6)$ & $0.0012(6)$ & $0.0004(5)$ \\
\hline $\mathrm{C} 426$ & $0.0250(7)$ & $0.0225(7)$ & $0.0212(7)$ & $0.0063(5)$ & $0.0001(5)$ & $0.0030(5)$ \\
\hline $\mathrm{C} 427$ & $0.0324(8)$ & $0.0313(8)$ & $0.0199(7)$ & $0.0023(6)$ & $-0.0023(6)$ & $0.0023(6)$ \\
\hline F471 & $0.0664(8)$ & $0.0348(6)$ & $0.0344(6)$ & $-0.0146(5)$ & $-0.0164(5)$ & $0.0025(5)$ \\
\hline F472 & $0.0479(7)$ & $0.0611(8)$ & $0.0335(6)$ & $0.0201(6)$ & $-0.0186(5)$ & $-0.0060(5)$ \\
\hline F473 & $0.0511(7)$ & $0.0703(8)$ & $0.0182(5)$ & $-0.0011(6)$ & $0.0044(4)$ & $-0.0003(5)$ \\
\hline
\end{tabular}

Geometric parameters $\left(\AA,{ }^{o}\right)$

\begin{tabular}{llll}
\hline $\mathrm{N} 1-\mathrm{C} 2$ & $1.3168(18)$ & $\mathrm{C} 32-\mathrm{C} 33$ & $1.511(2)$ \\
$\mathrm{N} 1-\mathrm{C} 8 \mathrm{~A}$ & $1.3720(18)$ & $\mathrm{C} 32-\mathrm{H} 32 \mathrm{~A}$ & 0.9900 \\
$\mathrm{C} 2-\mathrm{C} 3$ & $1.4292(18)$ & $\mathrm{C} 32-\mathrm{H} 32 \mathrm{~B}$ & 0.9900 \\
$\mathrm{C} 2-\mathrm{C} 21$ & $1.5021(19)$ & $\mathrm{C} 33-\mathrm{H} 33 \mathrm{~A}$ & 0.9800 \\
$\mathrm{C} 3-\mathrm{C} 4$ & $1.3786(18)$ & $\mathrm{C} 33-\mathrm{H} 33 \mathrm{~B}$ & 0.9800
\end{tabular}




\begin{tabular}{|c|c|c|c|}
\hline $\mathrm{C} 3-\mathrm{C} 31$ & $1.5014(18)$ & $\mathrm{C} 33-\mathrm{H} 33 \mathrm{C}$ & 0.9800 \\
\hline $\mathrm{C} 4-\mathrm{C} 4 \mathrm{~A}$ & $1.4322(18)$ & $\mathrm{C} 41-\mathrm{C} 42$ & $1.3388(19)$ \\
\hline $\mathrm{C} 4-\mathrm{C} 41$ & $1.4774(18)$ & $\mathrm{C} 41-\mathrm{H} 41$ & 0.9500 \\
\hline $\mathrm{C} 4 \mathrm{~A}-\mathrm{C} 5$ & $1.4191(19)$ & $\mathrm{C} 42-\mathrm{C} 421$ & $1.4701(18)$ \\
\hline $\mathrm{C} 4 \mathrm{~A}-\mathrm{C} 8 \mathrm{~A}$ & $1.4200(18)$ & $\mathrm{C} 42-\mathrm{H} 42$ & 0.9500 \\
\hline $\mathrm{C} 5-\mathrm{C} 6$ & $1.369(2)$ & $\mathrm{C} 421-\mathrm{C} 422$ & 1.3949 (19) \\
\hline $\mathrm{C} 5-\mathrm{H} 5$ & 0.9500 & $\mathrm{C} 421-\mathrm{C} 426$ & $1.400(2)$ \\
\hline $\mathrm{C} 6-\mathrm{C} 7$ & $1.409(2)$ & $\mathrm{C} 422-\mathrm{C} 423$ & 1.3867 (19) \\
\hline $\mathrm{C} 6-\mathrm{H} 6$ & 0.9500 & $\mathrm{C} 422-\mathrm{H} 422$ & 0.9500 \\
\hline $\mathrm{C} 7-\mathrm{C} 8$ & $1.368(2)$ & $\mathrm{C} 423-\mathrm{C} 424$ & $1.385(2)$ \\
\hline $\mathrm{C} 7-\mathrm{H} 7$ & 0.9500 & $\mathrm{C} 423-\mathrm{H} 423$ & 0.9500 \\
\hline $\mathrm{C} 8-\mathrm{C} 8 \mathrm{~A}$ & $1.4168(19)$ & $\mathrm{C} 424-\mathrm{C} 425$ & $1.389(2)$ \\
\hline $\mathrm{C} 8-\mathrm{H} 8$ & 0.9500 & $\mathrm{C} 424-\mathrm{C} 427$ & $1.497(2)$ \\
\hline $\mathrm{C} 21-\mathrm{H} 21 \mathrm{~A}$ & 0.9800 & $\mathrm{C} 425-\mathrm{C} 426$ & $1.387(2)$ \\
\hline $\mathrm{C} 21-\mathrm{H} 21 \mathrm{~B}$ & 0.9800 & $\mathrm{C} 425-\mathrm{H} 425$ & 0.9500 \\
\hline $\mathrm{C} 21-\mathrm{H} 21 \mathrm{C}$ & 0.9800 & $\mathrm{C} 426-\mathrm{H} 426$ & 0.9500 \\
\hline $\mathrm{C} 31-\mathrm{O} 31$ & $1.2087(17)$ & $\mathrm{C} 427-\mathrm{F} 472$ & $1.328(2)$ \\
\hline $\mathrm{C} 31-\mathrm{O} 32$ & $1.3342(17)$ & C427-F471 & $1.332(2)$ \\
\hline $\mathrm{O} 32-\mathrm{C} 32$ & $1.4614(16)$ & $\mathrm{C} 427-\mathrm{F} 473$ & $1.340(2)$ \\
\hline $\mathrm{C} 2-\mathrm{N} 1-\mathrm{C} 8 \mathrm{~A}$ & $118.08(12)$ & $\mathrm{C} 33-\mathrm{C} 32-\mathrm{H} 32 \mathrm{~A}$ & 109.3 \\
\hline $\mathrm{N} 1-\mathrm{C} 2-\mathrm{C} 3$ & $122.90(12)$ & $\mathrm{O} 32-\mathrm{C} 32-\mathrm{H} 32 \mathrm{~B}$ & 109.3 \\
\hline $\mathrm{N} 1-\mathrm{C} 2-\mathrm{C} 21$ & $116.74(12)$ & $\mathrm{C} 33-\mathrm{C} 32-\mathrm{H} 32 \mathrm{~B}$ & 109.3 \\
\hline $\mathrm{C} 3-\mathrm{C} 2-\mathrm{C} 21$ & $120.30(12)$ & $\mathrm{H} 32 \mathrm{~A}-\mathrm{C} 32-\mathrm{H} 32 \mathrm{~B}$ & 108.0 \\
\hline $\mathrm{C} 4-\mathrm{C} 3-\mathrm{C} 2$ & $120.52(12)$ & $\mathrm{C} 32-\mathrm{C} 33-\mathrm{H} 33 \mathrm{~A}$ & 109.5 \\
\hline $\mathrm{C} 4-\mathrm{C} 3-\mathrm{C} 31$ & $122.57(12)$ & $\mathrm{C} 32-\mathrm{C} 33-\mathrm{H} 33 \mathrm{~B}$ & 109.5 \\
\hline $\mathrm{C} 2-\mathrm{C} 3-\mathrm{C} 31$ & $116.42(11)$ & $\mathrm{H} 33 \mathrm{~A}-\mathrm{C} 33-\mathrm{H} 33 \mathrm{~B}$ & 109.5 \\
\hline $\mathrm{C} 3-\mathrm{C} 4-\mathrm{C} 4 \mathrm{~A}$ & $117.29(12)$ & $\mathrm{C} 32-\mathrm{C} 33-\mathrm{H} 33 \mathrm{C}$ & 109.5 \\
\hline $\mathrm{C} 3-\mathrm{C} 4-\mathrm{C} 41$ & $123.40(12)$ & $\mathrm{H} 33 \mathrm{~A}-\mathrm{C} 33-\mathrm{H} 33 \mathrm{C}$ & 109.5 \\
\hline $\mathrm{C} 4 \mathrm{~A}-\mathrm{C} 4-\mathrm{C} 41$ & $119.30(12)$ & $\mathrm{H} 33 \mathrm{~B}-\mathrm{C} 33-\mathrm{H} 33 \mathrm{C}$ & 109.5 \\
\hline $\mathrm{C} 5-\mathrm{C} 4 \mathrm{~A}-\mathrm{C} 8 \mathrm{~A}$ & $118.41(12)$ & $\mathrm{C} 42-\mathrm{C} 41-\mathrm{C} 4$ & $125.72(13)$ \\
\hline $\mathrm{C} 5-\mathrm{C} 4 \mathrm{~A}-\mathrm{C} 4$ & $123.25(12)$ & $\mathrm{C} 42-\mathrm{C} 41-\mathrm{H} 41$ & 117.1 \\
\hline $\mathrm{C} 8 \mathrm{~A}-\mathrm{C} 4 \mathrm{~A}-\mathrm{C} 4$ & $118.35(12)$ & $\mathrm{C} 4-\mathrm{C} 41-\mathrm{H} 41$ & 117.1 \\
\hline $\mathrm{C} 6-\mathrm{C} 5-\mathrm{C} 4 \mathrm{~A}$ & $120.57(13)$ & $\mathrm{C} 41-\mathrm{C} 42-\mathrm{C} 421$ & $124.22(13)$ \\
\hline $\mathrm{C} 6-\mathrm{C} 5-\mathrm{H} 5$ & 119.7 & $\mathrm{C} 41-\mathrm{C} 42-\mathrm{H} 42$ & 117.9 \\
\hline $\mathrm{C} 4 \mathrm{~A}-\mathrm{C} 5-\mathrm{H} 5$ & 119.7 & $\mathrm{C} 421-\mathrm{C} 42-\mathrm{H} 42$ & 117.9 \\
\hline $\mathrm{C} 5-\mathrm{C} 6-\mathrm{C} 7$ & $120.64(13)$ & $\mathrm{C} 422-\mathrm{C} 421-\mathrm{C} 426$ & $118.55(13)$ \\
\hline $\mathrm{C} 5-\mathrm{C} 6-\mathrm{H} 6$ & 119.7 & $\mathrm{C} 422-\mathrm{C} 421-\mathrm{C} 42$ & $122.19(13)$ \\
\hline $\mathrm{C} 7-\mathrm{C} 6-\mathrm{H} 6$ & 119.7 & $\mathrm{C} 426-\mathrm{C} 421-\mathrm{C} 42$ & $119.26(13)$ \\
\hline $\mathrm{C} 8-\mathrm{C} 7-\mathrm{C} 6$ & $120.48(13)$ & $\mathrm{C} 423-\mathrm{C} 422-\mathrm{C} 421$ & $120.76(13)$ \\
\hline $\mathrm{C} 8-\mathrm{C} 7-\mathrm{H} 7$ & 119.8 & $\mathrm{C} 423-\mathrm{C} 422-\mathrm{H} 422$ & 119.6 \\
\hline $\mathrm{C} 6-\mathrm{C} 7-\mathrm{H} 7$ & 119.8 & $\mathrm{C} 421-\mathrm{C} 422-\mathrm{H} 422$ & 119.6 \\
\hline $\mathrm{C} 7-\mathrm{C} 8-\mathrm{C} 8 \mathrm{~A}$ & $120.02(13)$ & $\mathrm{C} 424-\mathrm{C} 423-\mathrm{C} 422$ & $119.87(13)$ \\
\hline $\mathrm{C} 7-\mathrm{C} 8-\mathrm{H} 8$ & 120.0 & $\mathrm{C} 424-\mathrm{C} 423-\mathrm{H} 423$ & 120.1 \\
\hline $\mathrm{C} 8 \mathrm{~A}-\mathrm{C} 8-\mathrm{H} 8$ & 120.0 & $\mathrm{C} 422-\mathrm{C} 423-\mathrm{H} 423$ & 120.1 \\
\hline $\mathrm{N} 1-\mathrm{C} 8 \mathrm{~A}-\mathrm{C} 8$ & $117.31(12)$ & $\mathrm{C} 423-\mathrm{C} 424-\mathrm{C} 425$ & $120.37(13)$ \\
\hline $\mathrm{N} 1-\mathrm{C} 8 \mathrm{~A}-\mathrm{C} 4 \mathrm{~A}$ & $122.82(12)$ & $\mathrm{C} 423-\mathrm{C} 424-\mathrm{C} 427$ & $120.69(14)$ \\
\hline $\mathrm{C} 8-\mathrm{C} 8 \mathrm{~A}-\mathrm{C} 4 \mathrm{~A}$ & $119.87(12)$ & $\mathrm{C} 425-\mathrm{C} 424-\mathrm{C} 427$ & $118.94(14)$ \\
\hline
\end{tabular}




$\begin{array}{ll}\mathrm{C} 2-\mathrm{C} 21-\mathrm{H} 21 \mathrm{~A} & 109.5 \\ \mathrm{C} 2-\mathrm{C} 21-\mathrm{H} 21 \mathrm{~B} & 109.5 \\ \mathrm{H} 21 \mathrm{~A}-\mathrm{C} 21-\mathrm{H} 21 \mathrm{~B} & 109.5 \\ \mathrm{C} 2-\mathrm{C} 21-\mathrm{H} 21 \mathrm{C} & 109.5 \\ \mathrm{H} 21 \mathrm{~A}-\mathrm{C} 21-\mathrm{H} 21 \mathrm{C} & 109.5 \\ \mathrm{H} 21 \mathrm{~B}-\mathrm{C} 21-\mathrm{H} 21 \mathrm{C} & 109.5 \\ \mathrm{O} 31-\mathrm{C} 31-\mathrm{O} 32 & 124.46(13) \\ \mathrm{O} 31-\mathrm{C} 31-\mathrm{C} 3 & 123.44(12) \\ \mathrm{O} 32-\mathrm{C} 31-\mathrm{C} 3 & 111.98(11) \\ \mathrm{C} 31-\mathrm{O} 32-\mathrm{C} 32 & 116.34(11) \\ \mathrm{O} 32-\mathrm{C} 32-\mathrm{C} 33 & 111.65(11) \\ \mathrm{O} 32-\mathrm{C} 32-\mathrm{H} 32 \mathrm{~A} & 109.3 \\ & \\ \mathrm{C} 8 \mathrm{~A}-\mathrm{N} 1-\mathrm{C} 2-\mathrm{C} 3 & -1.0(2) \\ \mathrm{C} 8 \mathrm{~A}-\mathrm{N} 1-\mathrm{C} 2-\mathrm{C} 21 & 176.13(12) \\ \mathrm{N} 1-\mathrm{C} 2-\mathrm{C} 3-\mathrm{C} 4 & -1.0(2) \\ \mathrm{C} 21-\mathrm{C} 2-\mathrm{C} 3-\mathrm{C} 4 & -178.09(13) \\ \mathrm{N} 1-\mathrm{C} 2-\mathrm{C} 3-\mathrm{C} 31 & 171.16(13) \\ \mathrm{C} 21-\mathrm{C} 2-\mathrm{C} 3-\mathrm{C} 31 & -5.91(19) \\ \mathrm{C} 2-\mathrm{C} 3-\mathrm{C} 4-\mathrm{C} 4 \mathrm{~A} & 2.11(19) \\ \mathrm{C} 31-\mathrm{C} 3-\mathrm{C} 4-\mathrm{C} 4 \mathrm{~A} & -169.59(12) \\ \mathrm{C} 2-\mathrm{C} 3-\mathrm{C} 4-\mathrm{C} 41 & -178.53(12) \\ \mathrm{C} 31-\mathrm{C} 3-\mathrm{C} 4-\mathrm{C} 41 & 9.8(2) \\ \mathrm{C} 3-\mathrm{C} 4-\mathrm{C} 4 \mathrm{~A}-\mathrm{C} 5 & 178.82(13) \\ \mathrm{C} 41-\mathrm{C} 4-\mathrm{C} 4 \mathrm{~A}-\mathrm{C} 5 & -0.6(2) \\ \mathrm{C} 3-\mathrm{C} 4-\mathrm{C} 4 \mathrm{~A}-\mathrm{C} 8 \mathrm{~A} & -1.24(19) \\ \mathrm{C} 41-\mathrm{C} 4-\mathrm{C} 4 \mathrm{~A}-\mathrm{C} 8 \mathrm{~A} & 179.38(12) \\ \mathrm{C} 8 \mathrm{~A}-\mathrm{C} 4 \mathrm{~A}-\mathrm{C} 5-\mathrm{C} 6 & 0.7(2) \\ \mathrm{C} 4-\mathrm{C} 4 \mathrm{~A}-\mathrm{C} 5-\mathrm{C} 6 & -179.36(13) \\ \mathrm{C} 4 \mathrm{~A}-\mathrm{C} 5-\mathrm{C} 6-\mathrm{C} 7 & -0.4(2) \\ \mathrm{C} 5-\mathrm{C} 6-\mathrm{C} 7-\mathrm{C} 8 & -0.3(2) \\ \mathrm{C} 6-\mathrm{C} 7-\mathrm{C} 8-\mathrm{C} 8 \mathrm{~A} & 0.6(2) \\ \mathrm{C} 2-\mathrm{N} 1-\mathrm{C} 8 \mathrm{~A}-\mathrm{C} 8 & -178.55(13) \\ \mathrm{C} 2-\mathrm{N} 1-\mathrm{C} 8 \mathrm{~A}-\mathrm{C} 4 \mathrm{~A} & 1.9(2) \\ \mathrm{C} 7-\mathrm{C} 8-\mathrm{C} 8 \mathrm{~A}-\mathrm{N} 1 & -179.83(14) \\ \mathrm{C} 7-\mathrm{C} 8-\mathrm{C} 8 \mathrm{~A}-\mathrm{C} 4 \mathrm{~A} & -0.3(2) \\ \mathrm{C} 5-\mathrm{C} 4 \mathrm{~A}-\mathrm{C} 8 \mathrm{~A}-\mathrm{N} 1 & 179.16(13) \\ \mathrm{C} 4-\mathrm{C} 4 \mathrm{~A}-\mathrm{C} 8 \mathrm{~A}-\mathrm{N} 1 & -0.8(2) \\ \mathrm{C} 5-\mathrm{C} 4 \mathrm{~A}-\mathrm{C} 8 \mathrm{~A}-\mathrm{C} 8 & -0.3(2) \\ \mathrm{C} 4-\mathrm{C} 4 \mathrm{~A}-\mathrm{C} 8 \mathrm{~A}-\mathrm{C} 8 & 179.70(12) \\ \mathrm{C} 4-\mathrm{C} 3-\mathrm{C} 31-\mathrm{O} 31 & 72.26(19) \\ & \end{array}$

$$
\begin{aligned}
& \mathrm{C} 426-\mathrm{C} 425-\mathrm{C} 424 \\
& \text { C426-C } 425-\mathrm{H} 425 \\
& \mathrm{C} 424-\mathrm{C} 425-\mathrm{H} 425 \\
& \mathrm{C} 425-\mathrm{C} 426-\mathrm{C} 421 \\
& \mathrm{C} 425-\mathrm{C} 426-\mathrm{H} 426 \\
& \mathrm{C} 421-\mathrm{C} 426-\mathrm{H} 426 \\
& \text { F472-C427-F471 } \\
& \text { F472-C427-F473 } \\
& \text { F471-C } 427-\mathrm{F} 473 \\
& \text { F472-C } 427-\mathrm{C} 424 \\
& \text { F471-C } 427-\mathrm{C} 424 \\
& \text { F473-C } 427-\mathrm{C} 424
\end{aligned}
$$$$
\mathrm{C} 2-\mathrm{C} 3-\mathrm{C} 31-\mathrm{O} 31
$$$$
\mathrm{C} 4-\mathrm{C} 3-\mathrm{C} 31-\mathrm{O} 32
$$$$
\mathrm{C} 2-\mathrm{C} 3-\mathrm{C} 31-\mathrm{O} 32
$$$$
\mathrm{O} 31-\mathrm{C} 31-\mathrm{O} 32-\mathrm{C} 32
$$$$
\mathrm{C} 3-\mathrm{C} 31-\mathrm{O} 32-\mathrm{C} 32
$$$$
\mathrm{C} 31-\mathrm{O} 32-\mathrm{C} 32-\mathrm{C} 33
$$$$
\mathrm{C} 3-\mathrm{C} 4-\mathrm{C} 41-\mathrm{C} 42
$$$$
\mathrm{C} 4 \mathrm{~A}-\mathrm{C} 4-\mathrm{C} 41-\mathrm{C} 42
$$$$
\mathrm{C} 4-\mathrm{C} 41-\mathrm{C} 42-\mathrm{C} 421
$$$$
\mathrm{C} 41-\mathrm{C} 42-\mathrm{C} 421-\mathrm{C} 422
$$$$
\mathrm{C} 41-\mathrm{C} 42-\mathrm{C} 421-\mathrm{C} 426
$$$$
\mathrm{C} 426-\mathrm{C} 421-\mathrm{C} 422-\mathrm{C} 423
$$$$
\mathrm{C} 42-\mathrm{C} 421-\mathrm{C} 422-\mathrm{C} 423
$$$$
\mathrm{C} 421-\mathrm{C} 422-\mathrm{C} 423-\mathrm{C} 424
$$$$
\mathrm{C} 422-\mathrm{C} 423-\mathrm{C} 424-\mathrm{C} 425
$$$$
\mathrm{C} 422-\mathrm{C} 423-\mathrm{C} 424-\mathrm{C} 427
$$$$
\mathrm{C} 423-\mathrm{C} 424-\mathrm{C} 425-\mathrm{C} 426
$$$$
\mathrm{C} 427-\mathrm{C} 424-\mathrm{C} 425-\mathrm{C} 426
$$$$
\mathrm{C} 424-\mathrm{C} 425-\mathrm{C} 426-\mathrm{C} 421
$$$$
\mathrm{C} 422-\mathrm{C} 421-\mathrm{C} 426-\mathrm{C} 425
$$$$
\mathrm{C} 42-\mathrm{C} 421-\mathrm{C} 426-\mathrm{C} 425
$$$$
\mathrm{C} 423-\mathrm{C} 424-\mathrm{C} 427-\mathrm{F} 472
$$$$
\mathrm{C} 425-\mathrm{C} 424-\mathrm{C} 427-\mathrm{F} 472
$$$$
\mathrm{C} 423-\mathrm{C} 424-\mathrm{C} 427-\mathrm{F} 471
$$$$
\mathrm{C} 425-\mathrm{C} 424-\mathrm{C} 427-\mathrm{F} 471
$$$$
\text { C423-C424-C427-F473 }
$$$$
\text { C425-C424-C427-F473 }
$$

$119.56(14)$

120.2

120.2

120.83 (14)

119.6

119.6

$106.29(15)$

105.83 (14)

106.37 (14)

$113.19(14)$

111.94 (13)

$112.70(14)$

$-99.75(16)$

$-111.53(14)$

76.46 (15)

$4.82(19)$

$-171.35(11)$

-80.77 (15)

$34.8(2)$

-145.85 (14)

$-179.75(12)$

-17.7 (2)

$162.95(14)$

$-2.4(2)$

178.24 (13)

$0.3(2)$

$1.8(2)$

$-177.82(14)$

$-1.7(2)$

$177.92(14)$

$-0.5(2)$

2.5 (2)

$-178.13(14)$

8.1 (2)

$-171.53(15)$

$128.18(17)$

$-51.4(2)$

$-111.97(17)$

68.4 (2)

Hydrogen-bond geometry $\left(A,{ }^{\circ}\right)$

\begin{tabular}{lllll}
\hline$D-\mathrm{H} \cdots A$ & $D-\mathrm{H}$ & $\mathrm{H} \cdots A$ & $D \cdots A$ & $D-\mathrm{H} \cdots A$ \\
\hline $\mathrm{C} 41-\mathrm{H} 41 \cdots \mathrm{O} 31^{\mathrm{i}}$ & 0.95 & 2.54 & $3.4922(18)$ & 178
\end{tabular}


$\mathrm{C} 422-\mathrm{H} 422 \cdots \mathrm{O} 31^{\mathrm{i}}$

0.95

Symmetry code: (i) $-x+1,-y+1,-z+1$.
2.56

$3.3924(19)$

146 